\title{
Metabolic-scale gene activation screens identify SLCO2B1 as a heme transporter that enhances cellular iron availability
}

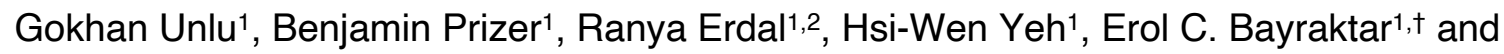 \\ Kıvanç Birsoy ${ }^{*}$
}

\begin{abstract}
'Laboratory of Metabolic Regulation and Genetics, The Rockefeller University, New York, NY, USA;
${ }^{2}$ Hacettepe University Medical School, Ankara, Turkey

† Present address: Department of Pathology, New York University School of Medicine, New York, NY, USA
\end{abstract}

*Corresponding and Lead Contact: kbirsoy@rockefeller.edu

\section{SUMMARY}

Iron is the most abundant transition metal in cells and essential for a wide range of biochemical processes. While most mammalian cells take up iron through receptor-mediated endocytosis of transferrin, molecular players involved in iron utilization under iron-limiting conditions are incompletely understood. To address this, we performed several parallel metabolism-focused CRISPRa gain of function screens, which revealed metabolic limitations under stress conditions. Screens for iron restriction identified expected members of iron utilization pathways, but also SLCO2B1, a poorly characterized membrane carrier. Expression of SLCO2B1 is sufficient to increase intracellular iron stores, bypass the essentiality of transferrin receptor-mediated iron uptake and enable cell proliferation under iron restriction. Mechanistically, SLCO2B1 mediates heme-analog import in cellular assays. Heme uptake by SLCO2B1 provides sufficient iron for cell proliferation through heme oxygenases. Notably, SLCO2B1 is predominantly expressed in microglia in the brain and primary microglia from Slco2b1/- mice exhibit a strong defect in heme analog import. Altogether, our work identifies SLCO2B1 as a microglia-enriched plasma membrane heme importer and provides a genetic platform to identify metabolic limitations under stress conditions. 


\section{INTRODUCTION}

Iron is the most abundant transition metal and essential for almost all living species. A significant fraction (2\%) of human proteins binds to iron in the form of heme (iron-protoporphyrin IX), iron-sulfur (FeS) clusters, and as free ion (Andreini et al., 2018; Kaplan and Ward, 2013). Owing to its unique redox characteristics, iron mediates a wide range of biological process including oxidative phosphorylation, DNA replication and antioxidant response. In mammals, iron deficiency causes anemia but is also associated with impaired neurological function and weakened immune response (Jiang et al., 2019; Yager and Hartfield, 2002). In contrast, excess cellular iron generates reactive oxygen species that damage cellular macromolecules, and is linked to aging, liver failure and neurodegeneration (Sato et al., 2022; Wang et al., 2017). Most mammalian cells take up iron in the form of transferrin, a serum glycoprotein that maintains it in soluble form and limits the generation of free radicals. However, iron also associates with other carriers in the serum such as ferritin, heme and albumin. These carriers are particularly critical under disease conditions where transferrin-bound iron levels are limiting (Leitner and Connor, 2012; Moos and Morgan, 1998). However, molecular players involved in iron utilization under iron-restricted conditions are incompletely understood.

To address this, we developed a metabolic scale CRISPR activation (CRISPRa) screening platform and performed gain-of-function genetic screens under iron-limiting conditions. These screens revealed multiple mechanisms that enable cell proliferation under iron restriction. Our work also identified $S L C O 2 B 1$, a poorly characterized plasma membrane transporter, as a heme transporter that promotes cellular iron availability. SLCO2B1 is predominantly expressed in microglia in the brain and primary microglia from S/co2 $\mathrm{b1}^{-/-}$mice exhibit a strong defect in heme analog import. Together, our results reveal metabolic limitations under iron restriction and identify a plasma membrane heme transporter in microglia.

\section{RESULTS}

\section{Metabolic-scale gene activation screens identify metabolic processes limiting for cell proliferation under iron restriction}

One approach to identify metabolic limitations under stress is to systematically induce the expression of small molecule transporters and enzymes and determine those that restore cell proliferation in response to a particular stress. Activation of target gene expression can be achieved with nuclease dead Cas9 (dCas9a) fused to a transcriptional activator (VP64-p65-Rta). Using this technology, we generated a metabolism focused sgRNA library consisting of 32,509 sgRNAs that target 2989 metabolic genes (Figure 1A). To test the robustness of the approach, we first performed gain-of-function genetic screens in dCas9a expressing PaTu-8988t pancreatic cancer cells (Figure S1A) treated with near-lethal doses of several metabolic inhibitors. These small molecules include Antimycin $A$, an electron transport chain (ETC) inhibitor, CB-839, an inhibitor of glutaminolysis, buthionine sulfoximine (BSO), an inhibitor of glutathione synthesis, and palmitate, a saturated fatty acid that disrupts membrane lipid homeostasis at high levels. After selection with these agents, we harvested DNA from surviving cell populations and calculated the percentage of total sgRNA reads mapping to each gene. Genes with highest percentage sgRNA reads protect cells from the corresponding toxic agents and reveal potential metabolic limitations for cell proliferation under these stress conditions.

Among the scoring genes that protect cells against ETC inhibition are SLC1A1 and SLC1A3, both of which are aspartate/glutamate plasma membrane transporters and increase aspartate import from the culture media. Indeed, previous work has determined that aspartate is a limiting metabolite for cancer cell proliferation under ETC inhibition (Birsoy et al., 2015). Interestingly, our screens also identified ASRGL1, a putative human L-asparaginase, which catalyzes the deamidation of asparagine to produce aspartate (Figure 1B). While ASRGL1 has previously been shown not to display amidase activity at physiological levels of expression (Pavlova et al., 2018), our data suggest that ASRGL1 may synthesize meaningful levels of aspartate when overexpressed in human cells. Conferring resistance to CB-839, our screens identify GLS2, a paralog of GLS1, as a gene that can bypass GLS1 inhibition, supporting the selective on-target effect of CB-839 on GLS1 (Figure 1C). SLC1A1/3 and ASRGL1 also scored likely due to their ability increase the cellular levels of glutamate, a limiting metabolite for TCA cycle progression 
under glutaminase inhibition. In line with the fact that BSO is a competitive glutamate analog (Meister, 1995), the only scoring gene in the BSO screen was glutamate-cysteine ligase catalytic subunit (GCLC), which catalyzes the first enzymatic step in glutathione (GSH) synthesis (Figure 1D). Finally, expression of FADS2 (fatty acid desaturase) and SCD (stearoyl-CoA desaturase), the two enzymes involved in fatty acid desaturation, protect cells against lipid saturation stress upon palmitate treatment (Figure 1E). Additional hits conferring resistance to palmitate were FADS1, which introduces cis $\triangle 5$ double bond during polyunsaturated fatty acid (PUFA) synthesis (Cho et al., 1999); ACSL4 ,the major enzyme that produces long chain PUFA containing lipids, ADIPOR2, which acts as a sensor for membrane fluidity and SLCO4A1, an uncharacterized transporter (Devkota et al., 2017; Ruiz et al., 2019) (Figure S1B). Notably, SLCO4A1 has previously been shown to be co-essential with enzymes in fatty acid metabolism, indicating its potential role in membrane lipid homeostasis (Aregger et al., 2020). These results highlight the utility of metabolism-scale gain of function screens to discover potential metabolic limitations under stress conditions.

We next sought to use this platform as a discovery tool to identify cellular pathways that regulate cellular iron availability under iron restriction. While iron chelators are commonly used to deplete cellular iron, we recently showed that disrupting lysosomal acidity also decreases cellular iron levels by hindering transferrin-mediated iron import (Weber et al., 2020). We therefore performed positive selection CRISPRa screens in Jurkat dCas9a cells (Figure S1A) and determined genes whose expression would overcome anti-proliferative effects of iron restriction induced by an iron chelator, deferoxamine mesylate (DFO, $2 \mu \mathrm{M}$ ), and a lysosomal pH inhibitor, Bafilomycin A1 (BafA1, $5 \mathrm{nM}$ ) (Figures 1F and 1G). Scoring genes should identify metabolic limitations under iron depletion and reveal alternative pathways involved in iron acquisition. Consistent with the observation that DFO and BafA1 impair cell proliferation via distinct mechanisms, most of the scoring genes were unique to each condition. Among the scoring genes in DFO screens are SLC25A37 and SLC25A28, mitochondrial iron importers (Shaw et al., 2006). Additionally, plasma membrane and endosomal ferric reductases that reduce $\mathrm{Fe}^{3+}$ to $\mathrm{Fe}^{2+}$ also scored, including CYBRD1, FRRS1 and CYB561A3 (Figure 1H) (Vargas et al., 2003; Wang et al., 2021). In contrast, genes conferring resistance to BafA1 included v-ATPase subunits ATP6VOA2 and ATPV1E1 among the top hits. These subunits are adjacent to the BafA1 binding site on the v-ATPase, and their overexpression likely blocks inhibition of v-ATPase activity by BafA1 (Figure 1I-J).

Iron plays essential roles in different compartments in cells. In addition to proteins containing heme or iron-sulfur (Fe-S) clusters, whose syntheses occur in the mitochondrial matrix, there are other key enzymes containing iron, but devoid of Fe-S clusters or heme. For example, di-iron center is present in a group of non-mitochondrial enzymes such as ribonucleotide reductases, oxygenases and fatty acid desaturases (Bollinger Jr et al., 1991; Ryle and Hausinger, 2002; Shen et al., 2020). Given that both mitochondrial iron transporters scored in our genetic screens, we hypothesized that iron availability in mitochondria, but not in the cytosol or other organelles, is the limiting process for cell proliferation under environmental iron restriction. Consistent with this hypothesis, overexpression of mitochondrial iron transporter SLC25A37 was sufficient to sustain cell proliferation at lethal DFO concentrations (Figures $1 \mathrm{~K}$ and $\mathbf{1 L}$ ). These data suggest that mitochondrial iron availability is the limiting process for proliferation when cells are deprived of iron. Altogether, our screens identify distinct metabolic players involved in cell survival and proliferation under iron chelators and lysosomal pH inhibition. Strikingly, SLCO2B1, a poorly characterized small molecule transporter, was the only common hit upon DFO and BafA1-induced iron restriction (Figures 1H, 1I, S1C, S1D and S1E). We therefore focused our attention on it.

\section{SLCO2B1 expression is sufficient to sustain cell proliferation under iron restriction}

SLCO2B1 is a member of organic anion transporter family (König, 2011) and has previously been shown to transport a multitude of drugs and steroid hormone conjugates (Grube et al., 2006; Medwid et al., 2019). However, its precise physiological substrate and relevance to iron metabolism is unknown. To begin to study its function, we generated a Jurkat cell line that expresses SLCO2B1 cDNA (Figure S1F). Consistent with the screen results, cells expressing SLCO2B1 can proliferate even under lethal doses of DFO and BafA1, in contrast to those expressing a control vector, its closely related paralog SLCO2A1 or 
a short SLCO2B1 isoform (Figures 2A, 2B, S1G, S1H). This finding is generalizable to other cell lines, as SLCO2B1 expression in PaTu-8988t cells protected them from DFO and BafA1-induced toxicity (Figures S1I and S1J). Additionally, positive selection CRISPRa screens in a mouse pancreas cancer cell line identified Slco2b1 as the top protective gene against lysosomal pH dysfunction (Figures S1K, S1L, S1M, S1N and S10). SLCO2B1-overexpressing cells displayed similar resistance to deferiprone (DFP), a lipid-soluble iron chelator, v-ATPase inhibitor concanamycin (ConA) and $\mathrm{NH}_{4} \mathrm{Cl}$, which disrupts lysosomal acidity by acting as a lysosomotropic weak base (Klempner and Styrt, 1983) (Figures 2C, 2D, 2E). These findings reveal that SLCO2B1 expression is sufficient to sustain proliferation of mammalian cells under different modes of iron depletion.

\section{SLCO2B1 promotes iron availability, independently of transferrin-mediated iron uptake in mammalian cells}

We next sought to determine the role of $S L C O 2 B 1$ in cellular iron metabolism. To address this in an unbiased way, we profiled cellular metabolites from Jurkat cells expressing SLCO2A1 (control) or SLCO2B1 cDNA by liquid chromatography-mass spectrometry (LC-MS). However, only few metabolites change in response to $S L C O 2 B 1$ overexpression, suggesting that SLCO2B1 expression does not cause a strong change in the levels of cellular metabolites (Figures S2A, S2B and S2C). Cellular iron homeostasis is tightly controlled in cells by a post-translational mechanism mediated by IRP1/2 pathway (Sviderskiy et al., 2019). An increase in iron levels negatively regulate IRP2, enhancing mRNA stability of ferritin heavy chain 1 (FTH1), the major iron storage protein in mammalian cells. To test whether SLCO2B1 modulates iron homeostasis, we determined IRP2 and FTH1 protein levels in cells expressing $S L C O 2 B 1$. Expression of SLCO2B1 caused a marked induction in the protein levels of FTH1 across several mammalian cell lines tested, under standard culture conditions (Figures 2F and S2D). We next asked whether SLCO2B1-expressing cells maintain higher levels of iron stores even under iron restriction. Indeed, expression of SLCO2B1 strongly blocked the increase in IRP2 and decrease in FTH1 protein levels in response to treatment with iron chelators or lysosomal pH inhibitors (Figure 2G). Finally, to directly measure iron levels in these cells, we conducted inductively coupled plasma mass spectrometry (ICP/MS) measurements. Consistent with the increase in ferritin levels, iron levels in SLCO2B1-expressing cells were 2 -fold higher than those of the parental controls. Notably, we did not observe any significant change in the levels of other divalent metals ( $\mathrm{Cu}, \mathrm{Mn}$ and $\mathrm{Zn}$ ), indicating a specific effect of SLCO2B1 on iron homeostasis (Figure 2H). These results suggest that SLC2OB1 regulates the iron response pathway by increasing cellular iron stores.

Under physiological conditions, TFRC-mediated iron uptake is the main route of iron acquisition (Richardson and Ponka, 1997). Indeed, TFRC is a universally essential gene for the proliferation of most cell lines (Tsherniak et al., 2017). To determine whether SLCO2B1 expression bypasses the requirement of TFRC-mediated iron uptake, we knocked out TFRC by CRISPR/Cas9 in Jurkat cells expressing SLCO2B1, its paralog SLCO2A1 or vector control (Figure S2E). Consistent with the essential function of TFRC for cell survival, parental Jurkat cells and those expressing SLC2OA1 died upon its depletion. Supplementation of culture media with excess free iron, i.e. ferric iron citrate (FAC), was sufficient to restore cell proliferation. In contrast, SLCO2B1 expression in Jurkat cells completely restored their survival and proliferation in response to TFRC loss (Figures $\mathbf{2 l}$ and $\mathbf{2} \mathbf{J}$ ). These results strongly suggest that SLCO2B1 promotes iron availability and cell proliferation under iron restriction, independently of transferrin-mediated iron uptake.

\section{Heme oxygenase is essential for SLCO2B1-induced resistance to iron restriction}

To understand how SLCO2B1 expression enables cell survival under iron restriction, we performed a negative selection CRISPR screen in parental Jurkat cells and those expressing SLCO2B1, treated with a normally lethal dose of BafA1. These screens should identify metabolic processes required for SLCO2B1-mediated rescue of iron restriction. Additionally, given that SLCO2B1-expressing cells tolerate high levels of BafA1 treatment, this provides a unique opportunity to determine metabolic processes that enable cell proliferation under severe lysosomal dysfunction (Figures 3A, 3B, S3A and 
S3B). Indeed, among the scoring genes differentially essential under lysosomal $\mathrm{pH}$ inhibition are those involved in cholesterol (LSS, SREBF2, ACAT2, SQLE, FDFT1) and sphingolipid (SPTLC1, SPTLC2 and SGMS1) biosynthesis. Cholesterol and sphingolipids can be synthesized de novo, but also acquired from extracellular lipoproteins through lysosomal enzymes. Similarly, cysteine import into lysosomes requires the lysosomal $\mathrm{pH}$ gradient. Likely due to the decrease in lysosomal cysteine storage, cells become dependent on cystine uptake mediated by SLC7A11 (Figures 3C and S3B) (Pisoni et al., 1990). Further work is needed to determine why several other processes scored as conditionally essential and how they may compensate for lysosomal dysfunction in mammalian cells.

Interestingly, the only scoring gene relevant to iron metabolism and differentially essential in SLCO2B1 expressing cells was HMOX2, a heme oxygenase (HMOX) required for heme degradation (Figures 3B, 3C, 3D and S3A). HMOX enzymes break down the porphyrin ring of heme to produce biliverdin, carbon monoxide and iron. Notably, while most mammalian cells express two HMOX paralogs, HMOX2 is the predominantly expressed one in Jurkat cells (TPM of HMOX1 = 0.3, HMOX2 = 32.8, DepMap portal). To test whether HMOX2 is necessary for SLCO2B1-mediated resistance against iron restriction, we deleted HMOX2 in parental and SLCO2A1 or SLCO2B1-expressing Jurkat cells. Consistent with the screen results, HMOX2 depletion completely abolished SLCO2B1-mediated resistance to BafA1 and DFO treatment (Figures 3E, 3F and S3C). Given the requirement of HMOX2 for SLCO2B1-mediated resistance, we reasoned that HMOX2 loss would block the increase in iron availability and the corresponding decrease in IRP2 protein abundance of SLCO2B1 expressing cells. In line with our model, HMOX2 loss restored IRP2 protein levels in SLCO2B1-expressing cells (Figure 3G). Collectively, these results suggest that HMOX2 acts downstream of SLCO2B1 to increase intracellular iron availability.

Given that heme degradation is required for SLC2OB1 function, we next sought to determine whether SLCO2B1 is involved in heme metabolism. Interestingly, when we compared gene essentialities between parental cells and those expressing $S L C O 2 B 1$, we found that UROD (uroporphyrinogen decarboxylase), like TFRC, is essential only in parental cells and not in SLCO2B1 expressing counterparts (Figures 3H, 3I, S3E, S3F). UROD is a universally essential enzyme in mammalian cells and catalyzes the third step of heme biosynthesis in the cytosol. We therefore asked whether SLCO2B1 expression may bypass the dependence of mammalian cells on de novo heme synthesis. While loss of $U R O D$ blocked the proliferation of parental Jurkat cells, expression of SLCO2B1 completely eliminated the anti-proliferative effects of UROD depletion (Figure 3J). Protein levels of ALAS1, the rate limiting enzyme of heme synthesis, is negatively regulated by cellular heme levels. In line with this, expression of SLCO2B1, but not control cDNAs, reduced the increase in ALAS1 protein levels in response to UROD depletion (Figure 3K and S3G). Similarly, SLC2OB1 expression blocked the increase in ALAS1 levels in cells treated with succinyl acetone, the pharmacological inhibitor of heme synthesis (Figures S3D and $\mathrm{S} 3 \mathrm{H})$. Altogether, these results indicate that $S L C O 2 B 1$ expression is sufficient to overcome heme synthesis deficiency.

\section{SLCO2B1 expression is necessary and sufficient for the uptake of heme analogs}

$S L C O 2 B 1$-expressing cells are remarkably resistant to the effects of iron restriction and heme synthesis deficiency, suggesting that SLCO2B1 may be a potential heme importer. To first determine the localization of SLCO2B1, we expressed in PaTu-8988t cells a C-terminally eGFP-tagged version of SLCO2B1, which is fully functional (Figures S4A, S4B, S4C and S4D). Confocal microscopy analysis of the SLCO2B1-eGFP fusion protein showed a strong plasma membrane localization (Figure S4A). To quantitatively measure steady state heme uptake, we used Zinc mesoporphyrin (ZnMP), a fluorescent heme analog (Figure 4A). We treated cells with ZnMP and measured intracellular fluorescence by flow cytometry. ZnMP uptake was markedly higher in SLCO2B1-expressing cells across three human cell lines tested (Jurkat 24h uptake; HepG2 and PaTu-8988t 15 min uptake assays) compared to parental controls (Figures 4B, 4C and 4D). To formally prove the direct cellular uptake, we next performed a ZnMP uptake assay in a time- and dose-dependent manner. During a 15-min dose-dependent uptake assay, S/co2b1-expressing cells took up significantly more ZnMP at all doses tested (e.g. $>5$ fold at 0.5 
$\mu \mathrm{M}$ ) (Figures 4E, 4F and S4E). Finally, since heme is a highly redox-active molecule, excess uptake of heme should be more toxic to cells that import it more efficiently. We therefore assayed sensitivity of SLCO2B1-expression and control cells to Hemin, a heme-like porphyrin structure with a ferric chloride center (Figure 4G). Indeed, SLCO2B1 expressing Jurkat cells displayed slower proliferation rates than control and SLCO2A1-expressing cells, in response to hemin treatment (Figure 4H). Remarkably, upon hemin addition, SLCO2B1-expressing cell pellets, in contrast to controls, displayed a discernably brown color, characteristic of hemin accumulation (Figure 4I). In parallel, hemin treatment resulted in greater reduction of ALAS1 levels in SLCO2B1-expressing cells, suggesting higher hemin availability and subsequent ALAS1 downregulation (Figure 4J). These results strongly suggest that SLCO2B1 mediates the transport of heme analogs.

We next sought to test whether SLCO2B1 is necessary for heme analog import. Notably, most cultured mammalian cells we have tested did not exhibit appreciable levels of SLCO2B1 expression. We therefore analyzed a publicly available single cell transcriptome dataset (Tabula Muris Consortium, 2018) to identify cell types with highest Slco2b1 expression (Figure 4K). Strikingly, S/co2b1 expression displays a highly restricted expression pattern in microglial cells. To study loss of function of S/co2b1, we therefore generated an Slco2b1-null mouse model (Figure S4F). These knockout mice are viable and enabled us to test the necessity of S/co2b1 for heme analog uptake in isolated microglial cells (Ledo et al., 2020) (Figure 4L). Cells dissociated from brain, other than microglia, did not display detectable ZnMP positivity (Figures 4M and 4N), suggesting that microglial cells could indeed be the predominant cell type with heme uptake activity in the brain. Remarkably, this ZnMP uptake activity in wild type microglia almost exclusively depends on S/CO2b1 expression. Indeed, microglia from S/co2b1 knockout mice display a strong reduction in ZnMP import compared to those from wild type controls (i.e. 86\% of wild type microglia, as opposed to $2.23 \%$ Slco2b1-null microglia, were ZnMP+) (Figure 40). These results suggest that Slco2b1 is necessary for heme analog uptake in microglia.

\section{DISCUSSION}

In this study, using a combination of genetic and biochemical strategies, we identify SLCO2B1 as a plasma membrane transporter that mediates heme import. SLCO2B1-mediated heme uptake bypasses two essential metabolic processes in mammalian cells: TFRC-mediated iron uptake and heme biosynthesis. Heme uptake by the heme-responsive paralogs, hrg-1 and hrg-4, has extensively been studied in the context of heme-auxotrophic C. elegans. Mammalian homolog of $\mathrm{hrg}-1$ facilitates lysosomal export of heme in splenic macrophages (Rajagopal et al., 2008; White et al., 2013) and hrg-4 in worms lacks a mammalian homolog. Furthermore, while previous work proposed FLVCR2 as a plasma membrane heme transporter, its ectopic expression failed to rescue heme-deficiency (Chambers et al., 2021; Duffy et al., 2010). Given that these transporters did not score in our genetic screens, SLCO2B1 may have the highest affinity for heme import among other putative heme importers. Notably, single cell transcriptomics data revealed that SLCO2B1 expression is highly enriched in microglia (Cao et al., 2020; Tabula Muris Consortium, 2018), indicating that SLCO2B1 might mediate microglial heme import. As free heme levels increase in disease conditions like hematoma and intracranial hemorrhage (Mracsko and Veltkamp, 2014; Vasconcellos et al., 2021), microglial heme uptake by SLCO2B1 could be a defense mechanism to protect neurons and astrocytes from oxidative heme damage. Furthermore, heme uptake may also be relevant in the context of neurodegenerative diseases, as induced HMOX1 expression and iron accumulation in microglial cells have been observed in Alzheimer's and Parkinson's disease (Fernández-Mendívil et al., 2021; Kenkhuis et al., 2021). Future work is needed to determine the precise role of microglial heme uptake in disease conditions. 


\section{EXPERIMENTAL MODEL AND SUBJECT DETAILS Mice}

All animal studies were performed according to a protocol approved by the Institutional Animal Care and Use Committee (IACUC) at Rockefeller University. Animals were housed in ventilated caging on a standard light-dark cycle with food and water ad libitum. The mouse strain C57BL/6N-

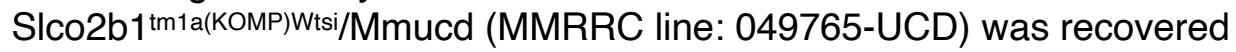
from cryopreserved sperm obtained from Mutant Mouse Resource and Research Center (MMRRC) at

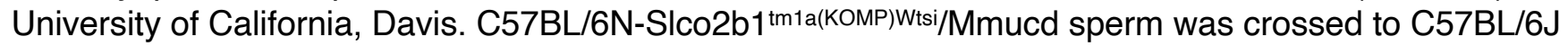
wild-type strain (from The Jackson Laboratory) through in

vitro fertilization at Rockefeller University Transgenic and Reproductive Technology Center. The progeny is maintained under standard conditions and identified through PCR amplification-based genotyping method provided by MMRRC. Homozygous mutant and homozygous wild type animals of 5-8 weeks were used in microglia isolation experiments. Genotypes were revealed to blinded investigators post-analysis.

\section{Cell Lines, Compounds and Constructs}

Jurkat, HepG2, HEK293T and MDA-MB-231 cell lines were purchased from ATCC. Other cell lines were kindly provided by the following investigators: PaTu-8988t pancreatic adenocarcinoma cell line by Dr. Monther Abu-Remaileh (Stanford University, CA), the HY15549 mouse pancreatic cancer line by Nabeel El-Bardeesy (Massachusetts General Hospital Cancer Center, MA), AK196 mouse pancreatic cancer line by Haoqiang Yang (MD Anderson Cancer Center, TX), 4T1 and 4T07 mouse breast cancer cell lines by Dr. Sohail Tavazoie (Rockefeller University, NY). All cell lines were verified to be mycoplasma contamination-free and authenticated by STR profiling. Reagents, compounds, and antibodies used in this study are listed in Table 1.

\section{Cell Culture Conditions}

Unless otherwise indicated, all listed cell lines, except for HepG2, were cultured in RPMI-1640 media (GIBCO) containing $2 \mathrm{mM}$ glutamine, $10 \%$ fetal bovine serum (SAFC, Sigma Aldrich) and 1\% penicillin and streptomycin (Invitrogen). HepG2 cells were cultured in DMEM (GIBCO) containing 4.5g/L glucose, $110 \mathrm{mg} / \mathrm{L}$ pyruvate, $4 \mathrm{mM}$ glutamine, supplemented with $10 \%$ fetal bovine serum and $1 \%$ penicillin and streptomycin. All cells were maintained at $37^{\circ} \mathrm{C}, 21 \% \mathrm{O}_{2}$ and $5 \% \mathrm{CO}_{2}$. For Figure S3, Jurkat cells were cultured in RPMI1640 supplemented with dialyzed FBS (GIBCO \#26400-044).

\section{METHOD DETAILS}

\section{Generation of overexpression and knockout constructs}

Gene fragments for coding sequences of human SLCA25A37, SLCO2B1, SLCO2A1; and mouse Slco2b1 were purchased from Twist Biosciences, then cloned into pLV-EF1a-IRES-Blast vector (Addgene \#85133) by Gibson assembly method. pLV-EF1a-IRES-Blast was used as a control vector control in overexpression experiments. Knockout cells were generated with CRISPR/Cas9 method. Forward and reverse oligos targeting TFRC, UROD and HMOX2 were annealed and ligated into BsmBI-linearized pLentiCRISPR v2 vector.

\section{Cell proliferation assays}

2,000 Jurkat cells or 500 PaTu-8988t or 500 mouse KPC cells/ per well were seeded, in triplicates, in $0.2 \mathrm{~mL}$ RPMI-1640 medium containing indicated treatments in 96-well plates.

On the day of seeding and the final day of treatment (as indicated in corresponding figures), $40 \mu \mathrm{L}$ of CellTiter-Glo reagent (Promega) was added, then, luminescence was measured on a SpectraMax M3 plate reader (Molecular Devices). Data are presented as cell doublings or the log2 fold change in luminescence on final treatment day compared to initial reading on the day of seeding. 


\section{Generation of knockout and overexpression cell lines}

For generation of knockout cells, VSV-G and Delta-VPR lentiviral packaging vectors were simultaneously transfected into HEK293T cells along with plentiCRISPR v2 vector expressing Cas9 and the gene-targeting sgRNA, using XtremeGene9 transfection reagent (Roche). Similarly, for overexpression, pLV-EF1a-IRES-Blast vector containing the gene-of-interest was transfected along with lentiviral packaging vectors VSV-G and Delta-VPR. $60 \mathrm{~h}$ post-transfection, the supernatant was collected after passing through a $0.45 \mu \mathrm{m}$ syringe filter. For transduction, $1 \times 10^{5}$ cells were plated in 6well plates containing $4 \mu \mathrm{g} / \mathrm{mL}$ polybrene and virus, and then spin-infected by centrifugation at 2,200 rpm for 80 minutes. Mixed population knockouts were selected with puromycin; overexpression cells were selected with blasticidin. Knockout and/or overexpression efficiency was assessed via immunoblotting. Jurkat or PaTu-8988t cells expressing dCas9-VPR were generated following a similar lentiviral transduction strategy. Instead, Edit-R Lentiviral CRISPRa dCas9-VPR vector (Horizon Discovery) was used as the overexpression vector. Single cell clones overexpressing dCas9-VPR were generated by FACS sorting single cells on a BD FACSAriall, 72-96 h post-infection into 96 well plates and grown for 2 weeks. Clones with strong overexpression were identified by Cas 9 immunoblotting.

\section{CRISPR/Cas9 genetic screens}

For metabolic scale CRISPRa screens in human cell lines, a metabolism-focused sgRNA library was designed and screens were performed as previously described (Birsoy et al., 2015; Wang et al., 2015). 2,989 metabolic genes were targeted with a total of 32,460 CRISPRa sgRNAs, whose designing approach was described previously (Horlbeck et al., 2016), and 49 non-targeting sgRNAs were included as controls. Oligonucleotides containing sgRNA sequences were synthesized by Agilent Technologies, PCR amplified and cloned into lentiGuide-Puro vector (Addgene \#52963). Briefly, amplicons were inserted into BsmBI-linearized lentiGuide-Puro vector by Gibson Assembly (NEB). Then, Gibson Assembly products were transformed into E. coli 10G SUPREME electrocompetent cells (Lucigen). This plasmid pool was used to produce lentivirus-containing supernatants in HEK293T cells. The titer of lentiviral supernatants was determined by infecting target cells at several amounts of virus in the presence of polybrene $(4 \mu \mathrm{g} / \mathrm{mL})$, counting the number of puromycin-resistant infected cells 3 days post-selection. For CRISPRa positive selection screens, 4 million target cells were infected at an MOI of $\sim 0.7$ and selected with puromycin $(4 \mu \mathrm{g} / \mathrm{mL}) 72 \mathrm{~h}$ post-infection. An initial pool of 4 million cells was harvested for genomic DNA extraction. The remaining cells were cultured for 14 doublings under specified drug treatment or untreated control conditions. On the final day of screening, cells were harvested for genomic DNA extraction. sgRNA inserts were PCR amplified, purified, and sequenced on MiSeq platform (Illumina). Sequencing reads were aligned to the library of sgRNA sequences and the abundance of each sgRNA was tallied. Results were reported as percentage of total sgRNA reads acquired per gene, gene score or differential gene score. Percent total sgRNA reads was calculated by summing up all sgRNA reads mapped to a gene and calculating the percentage of this sum to the number of sgRNA reads acquired from the entire population. sgRNA scores, representing the log2 fold change of the normalized final read count of the sgRNA from the initial read count of the sgRNA, were calculated. Gene score is defined as the median $\log _{2}$ fold change of all sgRNAs targeting the gene in the abundance of all sgRNAs targeting that gene, between the initial and final population. The differential gene score refers to the difference between the gene scores of drug-treated and untreated control groups.

For metabolic scale CRISPRa screens in mouse AK196 pancreas cell line, a metabolismfocused sgRNA library was designed and screens were performed similarly, as described above. For these screens, 1,839 metabolic genes were targeted with a total of 17,031 CRISPRa sgRNAs.

Metabolism-focused CRISPR knockout screens in human lines were performed as described previously (Zhu et al., 2019). $3 \times 10^{7}$ cells were infected with sgRNA library containing 23,971 sgRNAs targeting metabolic genes. Abundance of sgRNAs was quantified post-sequencing on NextSeq500 platform (Illumina). Full results of all CRISPR screens can be found in the supplemental tables S1-S15. 


\section{Metabolite Profiling}

For bulk metabolite profiling, $1 \times 10^{6}$ Jurkat cells, in triplicate, were grown for $24 \mathrm{~h}$ under DFO-, BafA1treatment or untreated conditions. Then, cells were rinsed in $0.9 \% \mathrm{NaCl}$ twice. Polar metabolites were extracted in $80 \%$ methanol containing ${ }^{15} \mathrm{~N}$ and ${ }^{13} \mathrm{C}$ fully-labeled amino acid standards (MSK-A2-1.2, Cambridge Isotope Laboratories, Inc). Extracts were shaken for $10 \mathrm{~min}$ with a vortexer, spun at 19,000 $\mathrm{g}$ to remove insoluble cell debris, nitrogen-dried and stored at $-80^{\circ} \mathrm{C}$ until liquid chromatography-mass spectrometry analysis (LC-MS). Then, LC-MS was performed as previously described (GarciaBermudez et al., 2018). Relative metabolite abundances were quantified using XCalibur QualBrowser 2.2 and Skyline Targeted Mass Spec Environment (MacCoss Lab) using a 5 ppm mass tolerance and a pooled-library of metabolite standards to verify metabolite identity. Relative metabolite levels were calculated by normalizing to total protein levels, as measured by Bicinchoninic Acid Assay (BCA).

\section{Measurement of Metals by Inductively Couple Plasma / Mass Spectrometry (ICP/MS)}

$3 \times 10^{7}$ Jurkat cells were seeded in triplicates and grown in standard culture conditions for $24 \mathrm{~h}$. Cell pellets were collected and stored at $-80^{\circ} \mathrm{C}$ until ICP/MS analysis. The cell pellet was resuspended in pure, distilled water of $\geq 80 \mu \mathrm{l}$. The cell pellet was lysed on ice, using 5 pulses with a cell disruptor/sonicator microtip. For each ICP/MS replicate, 30 to $50 \mu \mathrm{l}$ of cell lysate was digested using a 5:1 mixture of nitric acid (OPTIMA grade, 70\%, Fisher Scientific) and ultrapure hydrogen peroxide (ULTREX II, 30\%, Fisher Scientific). 30 lysate, $500 \mathrm{uL}$ of nitric acid and 100 uL hydrogen peroxide are added to $2 \mathrm{~mL}$ polypropylene tubes. This mixture was allowed to digest overnight at room temperature, heated at $95^{\circ} \mathrm{C}$ just until dry, and resuspended overnight in $1 \mathrm{ml}$ of $2 \%$ nitric acid for analysis. The mixture was mixed well the following day to ensure resuspension, and $800 \mu \mathrm{l}$ more $2 \%$ nitric acid is added along with $200 \mu \mathrm{l}$ of $10 \mathrm{X}$ internal standard (prepared in $2 \%$ nitric acid). For ICP/MS run, an Agilent 7900 ICP/MS instrument was operated in helium $(\mathrm{He})$ collision cell gas mode for all measurements. Elements were measured at the following isotopes: $56 \mathrm{Fe}, 55 \mathrm{Mn}, 63 \mathrm{Cu}, 66 \mathrm{Zn}$. Calibration standards and samples were prepared in an acid matrix of $2 \%$ OPTIMA Grade Nitric Acid. Solutions of Agilent Multi-element Calibration Standard 2A were prepared to obtain an eight-point calibration curve. Agilent Germanium (or Scandium) Standard(s) were added to calibration standards, blanks, and samples. Standards were used to correct for potential sample matrix and/or nebulization effects.

Amounts of metals within a sample were normalized by total amount of protein, as determined by BCA assay (Thermo Fisher Scientific).

\section{Immunoblotting}

$1 \times 10^{6}$ cells were washed in cold PBS and lysed in a buffer containing $10 \mathrm{mM}$ Tris- $\mathrm{HCl} \mathrm{pH} \mathrm{7.4,} 150$ $\mathrm{NaCl}, 1 \mathrm{mM}$ EDTA, 1\% Triton X-100, 2\% SDS, 0.1\% CHAPS, and protease inhibitors (Milipore Sigma). Lysates were sonicated, centrifuged at $1,000 \mathrm{~g}$, and supernatant was collcted as the protein lysate. Total protein quantified using BCA Protein Assay Kit (Thermo Fisher) with bovine serum albumin as a protein standard. Protein samples were resolved on $8 \%$ or $10 \%-20 \%$ SDS-PAGE gels (Novex, ThermoFisher) and analyzed by standard immunoblotting protocol. Briefly, PVDF membranes (Milipore) were incubated with primary antibodies at $4^{\circ} \mathrm{C}$ overnight. After washing off the primary antibodies in tris buffered saline/ $0.1 \%$ Tween-20 (TBS-T), secondary antibody incubation was performed at room temperature for $1 \mathrm{~h}$. Secondary antibodies including anti-mouse IgG-HRP linked (Cell Signaling, 7076) and anti-rabbit IgG-HRP linked (Cell Signaling, 7074), were used at 1:3,000 dilution. Blots were developed by ECL Chemiluminescent detection system (Perkin Elmer LLC) and film exposure. SRX101A Film Processor (Konica Minolta) and Premium autoradiography Films (Thomas Scientific) were used for developing.

\section{Immunocytochemistry and Confocal Microscopy}

PaTu-8988t cells were seeded on coverslips and grown overnight at $37^{\circ} \mathrm{C}$ with $5 \% \mathrm{CO}_{2}$. Next day, cells were fixed in $4 \%$ PFA at room temperature for $15 \mathrm{~min}$, permeabilized in $1 \%$ triton $\mathrm{X}-100$ in PBA, and then, blocked in $1 \%$ bovine serum albumin (Sigma) for $30 \mathrm{~min}$ at room temperature. Primary antibody 
incubation was performed overnight at $4^{\circ} \mathrm{C}$. Then, cells were rinsed in PBS twice and secondary antibody incubation was performed at room temperature for $30 \mathrm{~min}$. After washing twice in PBS, DAPI, nuclear counterstain, incubation was performed at room temperature for $10 \mathrm{~min}$, then cells were washed twice in PBS and mounted in ProLong Gold antifade mountant (Molecular Probes). Slides were imaged with Nikon A1R MP multiphoton microscope with confocal modality, using Nikon Plan Apo Y $60 \mathrm{X} / 1.40$ oil immersion objective.

\section{Real-time quantitative PCR}

Total RNA was isolated from mouse livers using TRIzol reagent (Thermo Fisher Scientific), following manufacturer's manual. After DNase I treatment (New England Biolabs), $1 \mu \mathrm{g}$ total RNA was used for cDNA synthesis with Superscript III RT kit (Invitrogen). qPCR was performed on a Thermo QuantStudio 6 Flex Real-Time PCR machine. The primer sequences were listed in Table 1. Gene expression levels were normalized to beta-actin using $\Delta \Delta$ Ct method.

\section{Zinc Mesoporphyrin (ZnMP) Uptake and Flow Cytometry Analysis}

Adherent cells (HepG2, PaTu-8988t and KP pancreas) were trypsinized and washed with 1X Hank's Balanced Salt Solution (HBSS) (Milipore Sigma) twice. $1 \times 10^{5}$ cells were placed in a microfuge tube and incubated with the indicated concentration of ZnMP (Frontier Scientific) in 1x HBSS for 15 min at room temperature. Uptake was terminated by placing cells on ice. Then, cell pellets were collected, washed twice in $1 \mathrm{X}$ HBSS and resuspended in cold FACS buffer (DPBS + 2\% FBS + 5mM EDTA). ZnMP uptake was assessed by Flow Cytometry (Attune NxT Flow Cytometer, Thermo Fisher). 10,000 events were recorded per each sample. ZnMP signal was detected by exciting with $561 \mathrm{~nm}$ laser, AlexaFluor 568 emission track was recorded as it overlaps with ZnMP emission. Data were analyzed and plotted with FCS Express 7 Research software, version 7.12 (De Novo Software, Inc).

For ZnMP uptake in Jurkat cells, $5 \mu \mathrm{M}$ ZnMP was diluted directly in culture media (RPMI $1640+$ $10 \% \mathrm{FBS})$. Cells were treated with ZnMP overnight at standard culture conditions $\left(37^{\circ} \mathrm{C}, 5 \% \mathrm{CO}_{2}\right)$. After that, Jurkat cells were washed twice in PBS and resuspended in FACS buffer. Flow cytometry and data analyses were carried out as described above.

For dose and time-dependent ZnMP uptake assays, mouse KP pancreas cells were used. Cells, in triplicate, were treated with 1:2 serial dilutions of ZnMP, between $0.125 \mu \mathrm{M}$ and $1 \mu \mathrm{M}$, including $0 \mu \mathrm{M}$ control (equal volume DMSO), for 15 minutes at room temperature. For time course uptake assay, cells, in triplicate, were treated with $1 \mu \mathrm{M}$ ZnMP for 0, 1, 2, 5 and 10 minutes at room temperature. Median fluorescence value of $\mathrm{ZnMP}^{+}$-gated population and number of gated events were multiplied to calculate total ZnMP fluorescence. Results were plotted in Prism 9 (GraphPad) as dose or time vs. total ZnMP fluorescence.

\section{Mouse microglia isolation and ZnMP uptake assay}

Mouse microglial cells were isolated from 8-10 week-old female mice as previously described (Ledo et al., 2020). Briefly, mice were sacrificed, whole brains were removed and placed in DPBS (Ca ${ }^{2+}$ and $\mathrm{Mg}^{2+}$ free) containing 5\% FBS and 1mM HEPES pH7.4. Brain tissue was minced with scissors and incubated in $4,000 \mathrm{U} / \mathrm{ml}$ of collagenase $\mathrm{D}$ (Roche) at $37^{\circ} \mathrm{C}$ for 30 minutes. Collagenase digestion was stopped by adding $10 \mathrm{mM}$ EDTA and incubating for additional 5 minutes at $37^{\circ} \mathrm{C}$. Digested tissue was passed through 70- $\mu$ m cell strainer, centrifuged at 2,000 rpm, washed in DPBS, and centrifuged in $38 \%$ Percoll gradient for $30 \mathrm{~min}$. Cell pellet containing microglia fraction was washed and resuspended in cold FACS buffer (DPBS + 2\% FBS + 5mM EDTA). Non-specific binding was blocked by incubation with Fc blocking antibody (BD Biosciences, Clone 2.4G2) for $15 \mathrm{~min}$. Cells were washed in FACS buffer and stained with Cx3cr1 antibody (Clone: SA011F11) conjugated to PE/Cy7. After that, cells were washed twice in FACS buffer to remove unbound, excess antibody. $5 \times 10^{5}$ cells were placed in a microfuge tube and incubated with $2 \mu \mathrm{M}$ ZnMP (Frontier Scientific) in 1x HBSS for 15 min at room temperature. Uptake was terminated by placing the cells on ice. Then, cell pellets were collected, washed twice in $1 \mathrm{X}$ HBSS and resuspended in cold FACS buffer (DPBS + $2 \%$ FBS + 5mM EDTA). 
ZnMP uptake was assessed by Flow Cytometry (Attune NxT Flow Cytometer, Thermo Fisher). 10,000 events were recorded per each sample. ZnMP signal was detected by exciting with $561 \mathrm{~nm}$ laser, AlexaFluor 568 emission track was recorded. Cx3cr1-PE/Cy7 signal was detected by exciting with 488 $\mathrm{nm}$ laser and recording emission at $780 \mathrm{~nm}$. Data were analyzed and plotted with FCS Express 7 Research software, version 7.12 (De Novo Software, Inc).

\section{ACKNOWLEDGEMENTS}

We thank all members of the Birsoy laboratory for helpful suggestions. G.U. is a Damon Runyon Fellow, supported by the Damon Runyon Cancer Research Foundation (DRG-2431-21), and by the grant UL1 TR001866 from the National Center for Advancing Translational Sciences (NCATS, National Institutes of Health (NIH) Clinical and Translational Science Award (CTSA) program. K.B. is supported by the NIH/NCI (DP2 OD024174-01), NIH/NIDDK (R01 DK123323-01), Pershing Square Sohn Foundation and Mark Foundation Emerging Leader Award; and is a Searle and Pew-Stewart Scholar.

\section{AUTHOR CONTRIBUTIONS}

Conceptualization, K.B. and G.U.; Methodology, G.U. and K.B.; Formal Analysis, G.U.; Investigation, G.U., B.P., R.E., H.-W.Y and E.C.B; Writing - Original Draft, G.U. and K.B.; Funding Acquisition, K.B. and G.U.

\section{DECLARATION OF INTERESTS}

K.B. is scientific advisor to Nanocare Pharmaceuticals and Barer Institute. Other authors declare no competing interests. 


\section{FIGURE LEGENDS}

Figure 1. Metabolic scale CRISPRa screens identify processes limiting for cell proliferation under iron restriction

(A) Scheme describing metabolism-focused positive selection CRISPRa screens.

(B-E) CRISPRa screen results for (B) Antimycin A (10 nM), (C) CB-839 (6 $\mu \mathrm{M})$, (D) BSO (2 mM) in PaTu 8988t dCas9a cells and (E) Palmitate $(200 \mu \mathrm{M})$ in Jurkat dCas9a cells. Data were plotted as genes in alphabetical order vs. percentage of sgRNAs reads per gene. Percent total sgRNA reads was calculated by summing up all sgRNA reads mapped to a gene and calculating the percentage of this sum to the number of sgRNA reads acquired from the entire population. Bubble size indicates number of detected sgRNAs post-screen.

(F) Maintaining iron availability is essential for cell proliferation. BafA1 and DFO decreases cellular iron availability through different mechanisms.

(G) Dose-dependent effects of DFO and BafA1 on proliferation of Jurkat cells (Mean $\pm S E M, n=5$ ).

(H) Gene scores of untreated vs. DFO $(2 \mu \mathrm{M})$-treated Jurkat-dCas9a cells (left). Gene score is the median $\log _{2}$ fold change in the abundance of all sgRNAs targeting that gene during the screening period. Differential gene scores of top 20 genes providing resistance to DFO (right). Iron/heme-related genes are indicated in dark blue.

(I) Gene scores of untreated vs. BafA1 (5 nM)-treated Jurkat-dCas9a cells (left). Differential gene scores of top 20 genes providing resistance to BafA1 (right). Vacuolar ATPase subunits are indicated in light blue, lipid metabolism-related genes in green, other genes in gray.

(J) Scheme displaying selected scoring genes conferring resistance to iron-restriction.

(K) Immunoblot analysis of control and SLC25A37-overexpressing Jurkat cells. $\beta$-actin was used as loading control.

(L) Fold change in the number $\left(\log _{2}\right)$ of control and SLC25A37-overexpressing cells after 5 days in the presence of indicated DFO concentrations (left). Representative bright-field micrographs of Jurkat cells after 5-day treatment with $3 \mu \mathrm{M}$ DFO (right). Scale bar is $50 \mu \mathrm{M}$.

\section{Figure 2. SLCO2B1 expression is sufficient to promote iron availability and sustain cell} proliferation under iron restriction

(A) Fold change in the number $\left(\log _{2}\right)$ of vector, SLCO2A1 and SLCO2B1-expressing cells after 5 days in the presence of indicated DFO concentrations (left). Representative bright-field micrographs of Jurkat cells after 5-day treatment with $3 \mu \mathrm{M}$ DFO (right). Scale bar is $50 \mu \mathrm{m}$.

(B) Fold change in the number $\left(\log _{2}\right)$ of vector, SLCO2A1 and SLCO2B1-expressing cells after 5 days in the presence of indicated BafA1 concentrations (left) and representative bright-field micrographs of Jurkat cells after 5-day treatment with $4 \mathrm{nM}$ BafA1 (right). Scale bar is $50 \mu \mathrm{m}$.

(C-E) Fold change in the number $\left(\log _{2}\right)$ of vector and SLCO2B1-expressing cells after 5 days in the presence of indicated (C) DFP, (D) $\mathrm{NH}_{4} \mathrm{Cl}$ and (E) ConA concentrations

(F) Schematic (top) showing IRP2-mediated regulation of ferritin heavy chain (FTH1). Increased iron levels inhibit IRP2, a negative regulator of FTH1 expression. Immunoblot analysis of FTH1 and SLCO2B1 in indicated human and mouse cell lines expressing vector or SLCO2B1 cDNA (bottom). $\beta$ actin was used as loading control.

(G) Immunoblot analysis of IRP2 and FTH1 in Jurkat and 293T cells expressing vector or SLCO2B1 CDNA, in the absence or presence of iron depleting agents BafA1 (3 nM in Jurkat; $10 \mathrm{nM}$ in 293T), $\mathrm{NH}_{4} \mathrm{Cl}$ (1 mM in Jurkat; $5 \mathrm{mM}$ in 293T) or DFO (3 $\mu \mathrm{M}$ in Jurkat; $100 \mu \mathrm{M}$ in 293T).

(H) Abundance of indicated metals in Jurkat cells expressing vector or SLCO2B1 cDNA. Results were normalized to total amount of protein and reported as 'pg metal per $\mu \mathrm{g}$ protein'.

(I) Representative bright-field micrographs of TFRC knockout Jurkat cells expressing vector, SLCO2A1 or SLCO2B1 cDNA. Scale bar is $50 \mu \mathrm{m}$.

(J) Fold change in the number $\left(\log _{2}\right)$ of $T F R C$ knockout Jurkat cells expressing a control vector, SLCO2A1 or SLCO2B1 cDNA without (top) or with $0.01 \mathrm{mg} / \mathrm{ml}$ ammonium ferric citrate (FAC) supplementation (bottom) after 3 days. (Mean \pm SEM, $n=3$, Student's t-test, $95 \%$ confidence interval). 
Figure 3. Heme oxygenase is essential for SLCO2B1-mediated resistance to iron restriction

(A) Schematic describing CRISPR loss of function screens in Jurkat cells expressing SLCO2B1 cDNA in the presence and absence of BafA1.

(B) Gene scores of untreated vs. BafA1-treated (4 nM) Jurkat cells expressing SLCO2B1 cDNA.

(C) Top 20 genes scoring as differentially essential upon BafA1 treatment in SLCO2B1-expressing cells compared to vector controls. Differential gene scores are plotted, and color matched with metabolic processes they are involved in, as listed on right.

(D) sgRNA scores of HMOX2-targeting sgRNAs in untreated and BafA1-treated Jurkat cells expressing SLCO2B1 cDNA.

(E) Schematic illustrating the proposed model for SLCO2B1-mediated rescue of cell proliferation under iron restriction.

(F) Fold change in the number $\left(\log _{2}\right)$ of control and HMOX2 knockout Jurkat cells expressing a control vector, SLCO2A1 or SLCO2B1 CDNA, during 5-day incubation with or without BafA1 at indicated concentrations. (Mean \pm SEM, $n=3$, Student's t-test, 95\% confidence interval).

(G) Immunoblot analysis for HMOX2 and IRP2 in control and HMOX2 knockout Jurkat cells expressing vector, SLCO2A1 or SLCO2B1 cDNA. GAPDH was used as loading control.

(H) Schematic depicting CRISPR loss of function screen in Jurkat cells expressing a vector control compared to those expressing SLCO2B1 cDNA.

(I) Gene scores of Jurkat cells expressing a control vector or SLCO2B1 cDNA.

(J) Fold change in the number $\left(\log _{2}\right)$ of control and UROD knockout Jurkat cells expressing vector, $S L C O 2 A 1$ or SLCO2B1 cDNA after 3 days. Cells were seeded 7 days after infecting with UROD sgRNA vector. (Mean \pm SEM, $n=3$, Student's t-test, 95\% confidence interval).

(K) Immunoblot analysis for UROD and ALAS1 in Jurkat cells expressing vector, SLCO2A1 or $S L C O 2 B 1$ cDNA infected with a control sgRNA or UROD sgRNA. GAPDH was used as loading control.

Figure 4. SLCO2B1 expression is necessary and sufficient for the uptake of heme analogs (A) Chemical structure of Zinc Mesoporphyrin (ZnMP).

(B-D) Flow cytometry analysis of ZnMP uptake in (B) Jurkat cells expressing vector, SLCO2A1 or $S L C O 2 B 1 \mathrm{cDNA}$, treated with $5 \mu \mathrm{M}$ ZnMP for $24 \mathrm{~h}(\mathrm{C}) \mathrm{HepG} 2$ cells expressing vector or SLCO2B1 cDNA treated with $1 \mu \mathrm{M}$ ZnMP for 15 min (D) PaTu-8988t cells expressing vector or SLCO2B1 cDNA treated with $2 \mu \mathrm{M}$ ZnMP for $15 \mathrm{~min}$.

(E) Dose curve for ZnMP uptake in mouse HY15549 pancreas cells expressing vector or Slco2b1 cDNA treated with indicated doses of ZnMP for 15 min at room temperature. (Mean $\pm S E M, n=3$, Student's t-test, 95\% confidence interval).

(F) Time course for ZnMP uptake in mouse HY15549 pancreas cells expressing vector or Slco2b1 cDNA treated with $1 \mu \mathrm{M}$ ZnMP for the indicated time points.

(G) Chemical structures of Heme B and Hemin.

$(\mathrm{H})$ Fold change in the number $\left(\log _{2}\right)$ of Jurkat cells expressing vector, SLCO2A1 or SLCO2B1 cDNA, during 5-day incubation with or without Hemin treatment at indicated concentrations. (Mean $\pm S E M, n=$ 3, Student's t-test, 95\% confidence interval).

(I) Representative images of cell pellets from Hemin-treated Jurkat cells expressing vector, SLCO2A1

or SLCO2B1 CDNA. Cells were treated with $30 \mu \mathrm{M}$ Hemin for $24 \mathrm{~h}$ prior to pelleting.

(J) Immunoblot analysis for ALAS1 in Succinyl Acetone (1mM) or Hemin (30 $\mu \mathrm{M})$ treated Jurkat cells expressing vector or SLCO2B1 CDNA. $\beta$-actin was used as loading control.

(K) t-SNE plot for S/co2b1 expression across single cell transcriptome of mouse cell types. Adapted from Tabula Muris.

(L) Experimental strategy for the ZnMP uptake assay of primary cells derived from brains of wild type and Slco2b1 knockout mice.

(M) Flow cytometry analysis of ZnMP fluorescence and Cx3cr1-labeling (microglia marker) in cells isolated from wild-type and S/co2b1 knockout mouse brains. 
bioRxiv preprint doi: https://doi.org/10.1101/2022.02.06.479330; this version posted February 7, 2022. The copyright holder for this preprint (which was not certified by peer review) is the author/funder. All rights reserved. No reuse allowed without permission.

(N) Flow cytometry analysis of ZnMP fluorescence in Cx3cr1- non-microglial cells (O) Flow cytometry analysis of ZnMP fluorescence in $\mathrm{Cx} 3 \mathrm{cr} 1^{+}$microglia 


\section{SUPPLEMENTARY FIGURE LEGENDS}

Figure S1. Positive selection CRISPRa screens reveal SLCO2B1 as the common top hit providing resistance to iron restriction in human and mouse cells

Related to Figures 1 and 2

(A) Immunoblot analysis for Cas9 expression in PaTu-8988t and Jurkat single cell clones. Clones highlighted in red were used for screens

(B) Top 15 genes scoring as protective against palmitate toxicity in Jurkat CRISPRa screens.

Differential gene scores (palmitate - untreated control) are plotted. Lipid metabolism-related genes are highlighted in orange.

(C) Gene score ranks from BafA1 and DFO CRISPRa screens in Jurkat cells. SLCO2B1 is highlighted as it has the lowest common rank, i.e. highest score.

(D) SLCO2B1 sgRNA scores in CRISPRa screens for DFO in Jurkat cells

(E) SLCO2B1 sgRNA scores in CRISPRa screens for BafA1 in Jurkat cells

(F) Immunoblot analysis for SLCO2B1 expression in Jurkat cells expressing vector or SLCO2B1 CDNA. $\beta$-actin was used as loading control.

(G) Fold change in the number $\left(\log _{2}\right)$ of vector, SLCO2B1 long isoform and SLCO2B1 short isoform expressing Jurkat cells after 5 days in the presence of indicated BafA1 concentrations.

$(\mathrm{H})$ Fold change in the number $\left(\log _{2}\right)$ of vector, SLCO2B1 long isoform and SLCO2B1 short isoform expressing Jurkat cells after 5 days in the presence of indicated DFO concentrations.

(I) Fold change in the number $\left(\log _{2}\right)$ of vector, SLCO2B1 long isoform and SLCO2B1 short isoform expressing PaTu-8988t cells after 5 days in the presence of indicated BafA1 concentrations.

(J) Fold change in the number $\left(\log _{2}\right)$ of vector, SLCO2B1 long isoform and SLCO2B1 short isoform expressing PaTu-8988t cells after 5 days in the presence of indicated DFO concentrations.

(K) Gene scores of untreated vs. BafA1 (15 nM)-treated mouse AK196 pancreas-dCas9a cells. Gene score is the median $\log _{2}$ fold change in the abundance of all sgRNAs targeting that gene during the screening period.

(L) Slco2b1 sgRNA scores in positive selection CRISPRa screens for BafA1 treatment in mouse AK196 pancreas cells

(M) Gene scores of untreated vs. ConA (1 nM)-treated mouse AK196 pancreas-dCas9a cells.

(N) Slco2b1 sgRNA scores in positive selection CRISPRa screens for ConA treatment in mouse AK196 pancreas cells

(O) Gene score ranks from BafA1 and ConA CRISPRa screens in mouse AK196 pancreas cells.

Slco2b1 is highlighted as the lowest common rank, i.e. highest score.

Figure S2. SLCO2B1 overexpression leads to an increase in cellular iron availability Related to Figure 2

(A-C) Volcano plots showing the $\log _{2}$ fold change in polar metabolite abundance between SLCO2B1 and SLCO2A1-expressing Jurkat cells vs. - $\log P$ values. The dotted lines on $\mathrm{x}$-axis indicate 2 -fold difference; that on $y$-axis represents $P=0.05$ (Student's t-test, two-tailed, 95\% confidence interval). (A) untreated, (B) DFO (3 $\mu \mathrm{M})$-treated and (C) BafA1 (3nM)-treated cells were assayed.

(D) Immunoblot analysis of FTH1 and SLCO2B1 in select human and mouse cell lines expressing vector or SLCO2B1 CDNA. $\beta$-actin was used as loading control.

(E) Immunoblot analysis of TFRC in TFRC knockout Jurkat cells expressing vector, SLCO2A1 or $S L C O 2 B 1$ cDNA. $\beta$-actin was included as loading control for immunoblots.

Figure S3. SLCO2B1 expression mediates resistance to iron restriction through heme oxygenase and overcomes heme deficiency

Related to Figure 3

(A) Gene score ranks from BafA1 screens in parental (3 nM BafA1) and SLCO2B1-expressing (4nM BafA1) Jurkat cells. Common hits essential in BafA1 treatment, regardless of the genotype, are in yellow highlighted area (genes scoring within top 32 in both screens). Genes that are only essential in 
SLCO2B1-overexpressing cells for BafA1-resistance are in green highlighted area. HMOX2, shown in green circle, is one of the top hits specifically in SLCO2B1-overexpressing cells.

(B) Top 50 genes scoring as differentially essential upon BafA1 treatment in SLCO2B1-expressing cells were grouped in metabolic pathways in which they are functioning.

(C) Fold change in the number $\left(\log _{2}\right)$ of $H M O X 2$ knockout Jurkat cells expressing vector, SLCO2A1 or $S L C O 2 B 1$ cDNA, during 5-day incubation with or without DFO at indicated concentrations. (Mean \pm SEM, $n=3$, Student's t-test, 95\% confidence interval).

(D) Schematic depicting key reacting in heme biosynthesis and heme degradation pathways. Succinyl acetone is a potent inhibitor of heme biosynthesis, targeting ALAS1.

(E) TFRC sgRNA scores in CRISPR knockout screens of Jurkat cells expressing a control vector or SLCO2B1 CDNA.

(F) UROD sgRNA scores in CRISPR knockout screens of Jurkat cells expressing a control vector or SLCO2B1 CDNA.

(G) Immunoblot analysis for UROD and ALAS1 in UROD knockout HepG2 cells expressing a control vector or SLCO2B1 cDNA. The position of ALAS1 band was shown with an arrow. $\beta$-actin was used as loading control.

(H) Immunoblot analysis of SLCO2B1 and ALAS1 in untreated control and $1 \mathrm{mM}$ succinyl acetonetreated HepG2 cells expressing a control vector or SLCO2B1 cDNA. $\beta$-actin was used as loading control.

\section{Figure S4. SLCO2B1 localizes to plasma membrane}

Related to Figure 4

(A) Confocal microscopy images of PaTu-8988t cells expressing SLCO2A1-eGFP or SLCO2B1-eGFP. Cell were immunostained for eGFP and LAMP1 (lysosomal marker). DAPI was used as nuclear counterstain. PM: plasma membrane.

(B) Immunoblot analysis for eGFP in PaTu-8988t cells expressing a control vector, SLCO2A1-eGFP or SLCO2B1-eGFP, either untreated or treated with indicated concentration of BafA1. $\beta$-actin was used as loading control.

(C) Fold change in the number $\left(\log _{2}\right)$ of PaTu-8988t cells expressing a control vector, SLCO2A1-eGFP or SLCO2B1-eGFP cDNA, during 5-day incubation with or without BafA1 at indicated concentrations.

(Mean \pm SEM, $n=3$, Student's t-test, 95\% confidence interval).

(D) Immunoblot analysis for eGFP and FTH1 in PaTu-8988t cells expressing a control vector, SLCO2A1-eGFP or SLCO2B1-eGFP cDNA. $\beta$-actin was used as loading control.

(E) Flow cytometry analysis of ZnMP uptake in mouse HY15549 pancreas cells expressing a control vector or Slco2b1 cDNA, treated with1 $\mu \mathrm{M}$ ZnMP for $15 \mathrm{~min}$. ZnMP+ gate shown here was applied in dose-dependent ZnMP uptake assays

(F) Relative expression levels of S/co2b1 and S/co2a1 genes in wild-type (+/+), heterozygous (+/-) and homozygous mutant (+/-) mice from Slco2b1tm1a mouse line. Expression levels were normalized to beta-actin. Specific loss of Sloc2b1 expression in homozygous mutants was observed; Slco2a1 expression was assayed as a control. (Mean \pm SEM, $n=3$, Student's t-test, 95\% confidence interval). 


\section{Table 1. Reagents and resources}

\section{SUPPLEMENTARY TABLES}

Table S1. Antimycin A CRISPRa screen results in PaTu-8988t cells

Table S2. CB-839 CRISPRa screen results in PaTu-8988t cells

Table S3. BSO CRISPRa screen results in PaTu-8988t cells

Table S4. Palmitate CRISPRa screen results in Jurkat cells

Table S5. Differential Gene Scores in Palmitate CRISPRa screen performed in Jurkat cells Table S6. Gene Scores from Jurkat CRISPRa screens for DFO

Table S7. Differential Gene Scores in DFO CRISPRa screen performed in Jurkat cells

Table S8. Gene Scores from Jurkat CRISPRa screens for BafA1

Table S9. Differential Gene Scores in BafA1 CRISPRa screen performed in Jurkat cells

Table S10. Gene Scores from CRISPRa screens for BafA1 in mouse AK196 pancreas cells

Table S11. Gene Scores from CRISPRa screens for ConA in mouse AK196 pancreas cells

Table S12. Gene Scores from CRISPR knockout screens for BafA1 in SLCO2B1-expressing Jurkat cells

Table S13. Differential Gene Scores from CRISPR knockout screens for BafA1 in SLCO2B1expressing Jurkat cells

Table S14. Gene Scores from CRISPR knockout screens for BafA1 in parental Jurkat cells Table S15. Gene Scores from CRISPR knockout screens for differential essentiality of genes between control vector and SLCO2B1-expressing Jurkat cells 


\section{REFERENCES}

Andreini, C., Putignano, V., Rosato, A., and Banci, L. (2018). The human iron-proteome. Metallomics : integrated biometal science 10. 10.1039/c8mt00146d.

Aregger, M., Lawson, K.A., Billmann, M., Costanzo, M., Tong, A.H.Y., Chan, K., Rahman, M., Brown, K.R., Ross, C., Usaj, M., et al. (2020). Systematic mapping of genetic interactions for de novo fatty acid synthesis identifies C12orf49 as a regulator of lipid metabolism. Nature metabolism 2. 10.1038/s42255020-0211-z.

Birsoy, K., Wang, T., Chen, W., Freinkman, E., Abu-Remaileh, M., and Sabatini, D.M. (2015). An essential role of the mitochondrial electron transport chain in cell proliferation is to enable aspartate synthesis. Cell 162, 540-551. 10.1016/j.cell.2015.07.016.

Bollinger Jr, J.M., Edmondson, D.E., Huynh, B.H., Filley, J., Norton, J.R., and Stubbe, J. (1991). Mechanism of assembly of the tyrosyl radical-dinuclear iron cluster cofactor of ribonucleotide reductase. Science (New York, N.Y.) 253. 10.1126/science.1650033.

Cao, J., O'Day, D.R., Pliner, H.A., Kingsley, P.D., Deng, M., Daza, R.M., Zager, M.A., Aldinger, K.A., Blecher-Gonen, R., Zhang, F., et al. (2020). A human cell atlas of fetal gene expression. Science (New York, N.Y.) 370. 10.1126/science.aba7721.

Chambers, I.G., Willoughby, M.M., Hamza, I., and Reddi, A.R. (2021). One ring to bring them all and in the darkness bind them: The trafficking of heme without deliverers. Biochimica et biophysica acta. Molecular cell research 1868. 10.1016/j.bbamcr.2020.118881.

Cho, H.P., Nakamura, M., and Clarke, S.D. (1999). Cloning, expression, and fatty acid regulation of the human delta-5 desaturase. The Journal of biological chemistry 274. 10.1074/jbc.274.52.37335.

Devkota, R., Svensk, E., Ruiz, M., Ståhlman, M., Borén, J., and Pilon, M. (2017). The adiponectin receptor AdipoR2 and its Caenorhabditis elegans homolog PAQR-2 prevent membrane rigidification by exogenous saturated fatty acids. PLoS Genetics 13. 10.1371/journal.pgen.1007004.

Duffy, S.P., Shing, J., Saraon, P., Berger, L.C., Eiden, M.V., Wilde, A., and Tailor, C.S. (2010). The Fowler syndrome-associated protein FLVCR2 is an importer of heme. Molecular and cellular biology 30. 10.1128/MCB.00690-10.

Fernández-Mendívil, C., Luengo, E., Trigo-Alonso, P., García-Magro, N., Negredo, P., and López, M.G. (2021). Protective role of microglial HO-1 blockade in aging: Implication of iron metabolism. Redox biology 38. 10.1016/j.redox.2020.101789.

Garcia-Bermudez, J., Baudrier, L., La, K., Zhu, X.G., Fidelin, J., Sviderskiy, V.O., Papagiannakopoulos, T., Molina, H., Snuderl, M., Lewis, C.A., et al. (2018). Aspartate is a limiting metabolite for cancer cell proliferation under hypoxia and in tumours. Nat Cell Biol 20, 775-781. 10.1038/s41556-018-0118-z.

Grube, M., Köck, K., Karner, S., Reuther, S., Ritter, C.A., Jedlitschky, G., and Kroemer, H.K. (2006). Modification of OATP2B1-mediated transport by steroid hormones. Molecular pharmacology 70. 10.1124/mol.106.026450.

Horlbeck, M.A., Gilbert, L.A., Villalta, J.E., Adamson, B., Pak, R.A., Chen, Y., Fields, A.P., Park, C.Y., Corn, J.E., Kampmann, M., and Weissman, J.S. (2016). Compact and highly active next-generation libraries for CRISPR-mediated gene repression and activation. eLife 5. 10.7554/eLife.19760. 
Jiang, Y., Li, C., Wu, Q., An, P., Huang, L., Wang, J., Chen, C., Chen, X., Zhang, F., Ma, L., et al. (2019). Iron-dependent histone 3 lysine 9 demethylation controls B cell proliferation and humoral immune responses. Nature communications 10. 10.1038/s41467-019-11002-5.

Kaplan, J., and Ward, D.M. (2013). The essential nature of iron usage and regulation. Current Biology 23. 10.1016/j.cub.2013.05.033.

Kenkhuis, B., Somarakis, A., de Haan, L., Dzyubachyk, O., IJsselsteijn, M.E., de Miranda, N.F.C.C., Lelieveldt, B.P.F., Dijkstra, J., van Roon-Mom, W.M.C., Höllt, T., and van der Weerd, L. (2021). Iron loading is a prominent feature of activated microglia in Alzheimer's disease patients. Acta Neuropathologica Communications 9. 10.1186/s40478-021-01126-5.

Klempner, M.S., and Styrt, B. (1983). Alkalinizing the intralysosomal pH inhibits degranulation of human neutrophils. The Journal of clinical investigation 72. 10.1172/JCl111139.

König, J. (2011). Uptake transporters of the human OATP family: molecular characteristics, substrates, their role in drug-drug interactions, and functional consequences of polymorphisms. In Handbook of Experiemental Pharmacology, (Handb Exp Pharmacol). 10.1007/978-3-642-14541-4_1.

Ledo, J.H., Zhang, R., Mesin, L., Mourão-Sá, D., Azevedo, E.P., Troyanskaya, O.G., Bustos, V., and Greengard, P. (2020). Lack of a site-specific phosphorylation of Presenilin 1 disrupts microglial gene networks and progenitors during development. PloS one 15. 10.1371/journal.pone.0237773.

Leitner, D.F., and Connor, J.R. (2012). Functional roles of transferrin in the brain. Biochimica et Biophysica Acta 1820. 10.1016/j.bbagen.2011.10.016.

Medwid, S., Li, M.M.J., Knauer, M.J., Lin, K., Mansell, S.E., Schmerk, C.L., Zhu, C., Griffin, K.E., Yousif, M.D., Dresser, G.K., et al. (2019). Fexofenadine and Rosuvastatin Pharmacokinetics in Mice with Targeted Disruption of Organic Anion Transporting Polypeptide 2B1. Drug metabolism and disposition: the biological fate of chemicals 47. 10.1124/dmd.119.087619.

Meister, A. (1995). Mitochondrial changes associated with glutathione deficiency. Biochimica et biophysica acta 1271. 10.1016/0925-4439(95)00007-q.

Moos, T., and Morgan, E.H. (1998). Evidence for low molecular weight, non-transferrin-bound iron in rat brain and cerebrospinal fluid. Journal of Neuroscience Research 54. 10.1002/(SICl)10974547(19981115)54:4<486::AID-JNR6>3.0.CO;2-I.

Mracsko, E., and Veltkamp, R. (2014). Neuroinflammation after intracerebral hemorrhage. Frontiers in cellular neuroscience 8. 10.3389/fncel.2014.00388.

Pavlova, N.N., Hui, S., Ghergurovich, J.M., Fan, J., Intlekofer, A.M., White, R.M., Rabinowitz, J.D., Thompson, C.B., and Zhang, J. (2018). As Extracellular Glutamine Levels Decline, Asparagine Becomes an Essential Amino Acid. Cell metabolism 27. 10.1016/j.cmet.2017.12.006.

Pisoni, R.L., Acker, T.L., Lisowski, K.M., Lemons, R.M., and Thoene, J.G. (1990). A cysteine-specific lysosomal transport system provides a major route for the delivery of thiol to human fibroblast lysosomes: possible role in supporting lysosomal proteolysis. The Journal of Cell Biology 110. 10.1083/jcb.110.2.327.

Rajagopal, A., Rao, A.U., Amigo, J., Tian, M., Upadhyay, S.K., Hall, C., Uhm, S., Mathew, M.K., Fleming, M.D., Paw, B.H., et al. (2008). Haem homeostasis is regulated by the conserved and concerted functions of HRG-1 proteins. Nature 453. 10.1038/nature06934. 
Richardson, D.R., and Ponka, P. (1997). The molecular mechanisms of the metabolism and transport of iron in normal and neoplastic cells. Biochimica et biophysica acta 1331. 10.1016/s03044157(96)00014-7.

Ruiz, M., Ståhlman, M., Borén, J., and Pilon, M. (2019). AdipoR1 and AdipoR2 maintain membrane fluidity in most human cell types and independently of adiponectin. Journal of lipid research 60. 10.1194/jlr.M092494.

Ryle, M.J., and Hausinger, R.P. (2002). Non-heme iron oxygenases. Current opinion in chemical biology 6. 10.1016/s1367-5931(02)00302-2.

Sato, T., Shapiro, J.S., Chang, H.C., Miller, R.A., and Ardehali, H. (2022). Aging is associated with increased brain iron through cortex-derived hepcidin expression. eLife 11. 10.7554/eLife.73456.

Shaw, G.C., Cope, J.J., Li, L., Corson, K., Hersey, C., Ackermann, G.E., Gwynn, B., Lambert, A.J., Wingert, R.A., Traver, D., et al. (2006). Mitoferrin is essential for erythroid iron assimilation. Nature 440. 10.1038/nature04512.

Shen, J., Wu, G., Tsai, A.L., and Zhou, M. (2020). Structure and Mechanism of a Unique Diiron Center in Mammalian Stearoyl-CoA Desaturase. Journal of molecular biology 432. 10.1016/j.jmb.2020.05.017.

Sviderskiy, V.O., Terzi, E.M., and Possemato, R. (2019). Iron-Sulfur Cluster Metabolism Impacts Iron Homeostasis, Ferroptosis Sensitivity, and Human Disease. In Ferroptosis in Health and Disease., (Springer, Cham). https://doi.org/10.1007/978-3-030-26780-3 12.

Tabula Muris Consortium (2018). Single-cell transcriptomics of 20 mouse organs creates a Tabula Muris. Nature 562. 10.1038/s41586-018-0590-4.

Tsherniak, A., Vazquez, F., Montgomery, P.G., Weir, B.A., Kryukov, G., Cowley, G.S., Gill, S., Harrington, W.F., Pantel, S., Krill-Burger, J.M., et al. (2017). Defining a Cancer Dependency Map. Cell 170. 10.1016/j.cell.2017.06.010.

Vargas, J.D., Herpers, B., McKie, A.T., Gledhill, S., McDonnell, J., van den Heuvel, M., Davies, K.E., and Ponting, C.P. (2003). Stromal cell-derived receptor 2 and cytochrome b561 are functional ferric reductases. Biochimica et biophysica acta 1651. 10.1016/s1570-9639(03)00242-5.

Vasconcellos, L.R.C., Martimiano, L., Dantas, D.P., Fonseca, F.M., Mata-Santos, H., Travassos, L., Mendez-Otero, R., Bozza, M.T., and Pimentel-Coelho, P.M. (2021). Intracerebral Injection of Heme Induces Lipid Peroxidation, Neuroinflammation, and Sensorimotor Deficits. Stroke 52.

10.1161/STROKEAHA.120.031911.

Wang, H., An, P., Xie, E., Wu, Q., Fang, X., Gao, H., Zhang, Z., Li, Y., Wang, X., Zhang, J., et al. (2017). Characterization of ferroptosis in murine models of hemochromatosis. Hepatology (Baltimore, Md.) 66. 10.1002/hep.29117.

Wang, T., Birsoy, K., Hughes, N.W., Krupczak, K.M., Post, Y., Wei, J.J., Lander, E.S., and Sabatini, D.M. (2015). Identification and characterization of essential genes in the human genome. Science 350. 10.1126/science.aac7041.

Wang, Z., Guo, R., Trudeau, S.J., Wolinsky, E., Ast, T., Liang, J.H., Jiang, C., Ma, Y., Teng, M., Mootha, V.K., and Gewurz, B.E. (2021). CYB561A3 is the key lysosomal iron reductase required for Burkitt B-cell growth and survival. Blood 138. 10.1182/blood.2021011079. 
Weber, R.A., Yen, F.S., Nicholson, S.P.V., Alwaseem, H., Bayraktar, E.C., Alam, M., Timson, R.C., La, K., Abu-Remaileh, M., Molina, H., and Birsoy, K. (2020). Maintaining Iron Homeostasis Is the Key Role of Lysosomal Acidity for Cell Proliferation. Mol Cell. 10.1016/j.molcel.2020.01.003.

White, C., Yuan, X., Schmidt, P.J., Bresciani, E., Samuel, T.K., Campagna, D., Hall, C., Bishop, K., Calicchio, M.L., Lapierre, A., et al. (2013). HRG1 is essential for heme transport from the phagolysosome of macrophages during erythrophagocytosis. Cell metabolism 17. 10.1016/j.cmet.2013.01.005.

Yager, J.Y., and Hartfield, D.S. (2002). Neurologic manifestations of iron deficiency in childhood. Pediatric neurology 27. 10.1016/s0887-8994(02)00417-4.

Zhu, X.G., Nicholson Puthenveedu, S., Shen, Y., La, K., Ozlu, C., Wang, T., Klompstra, D., Gultekin, Y., Chi, J., Fidelin, J., et al. (2019). CHP1 Regulates Compartmentalized Glycerolipid Synthesis by Activating GPAT4. Mol Cell 74, 45-58.e47. 10.1016/j.molcel.2019.01.037. 
A $\quad \begin{gathered}\text { Metabolism-focused } \\ \text { CRISPRa sgRNA library }\end{gathered}$

(32,509 sgRNAs)

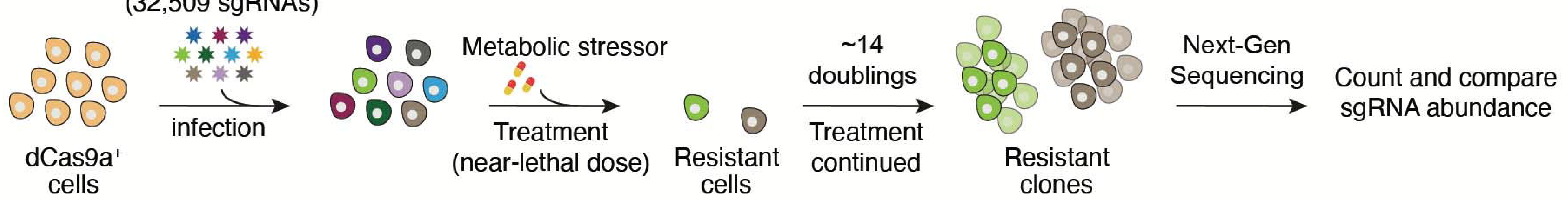

B

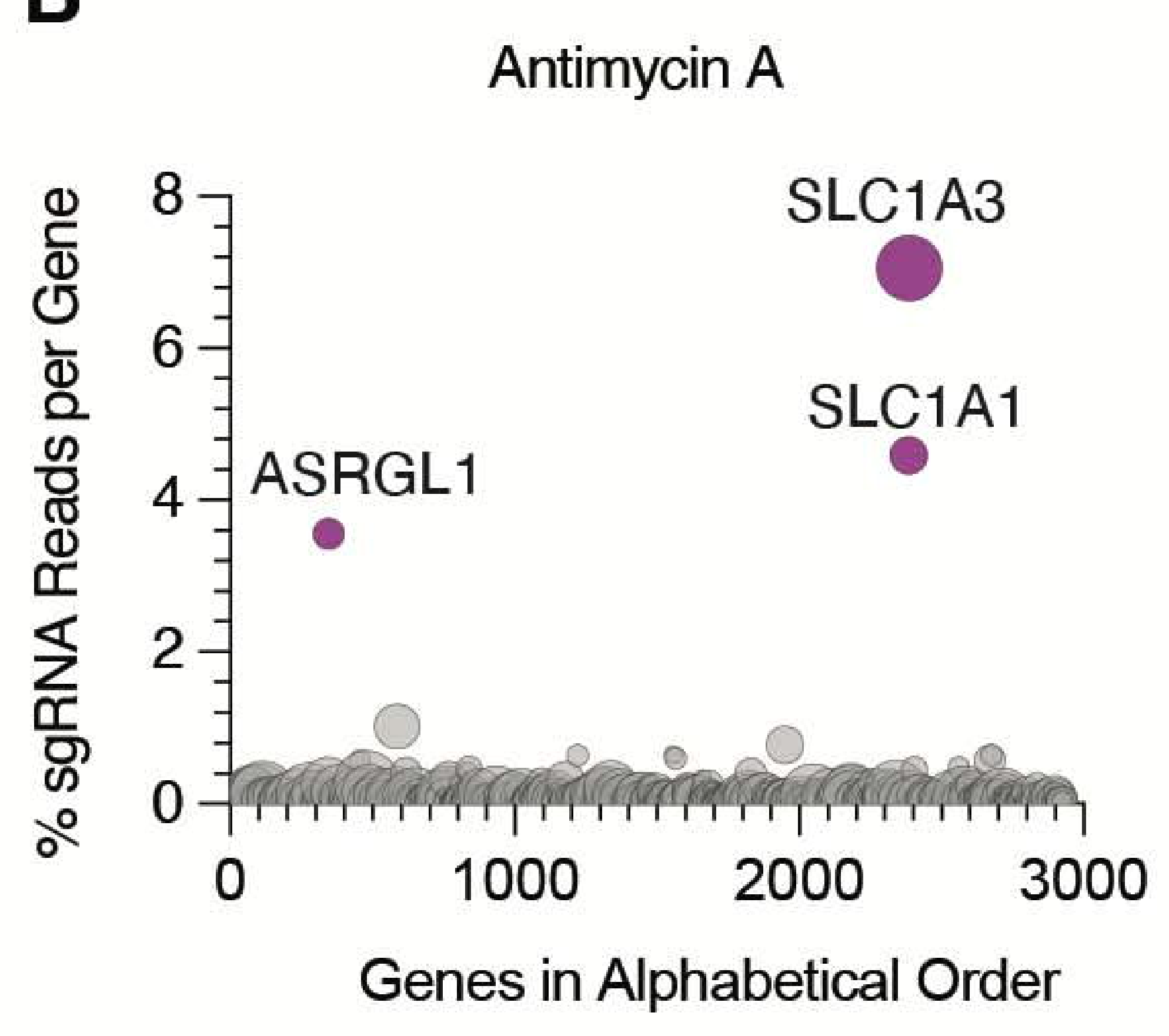

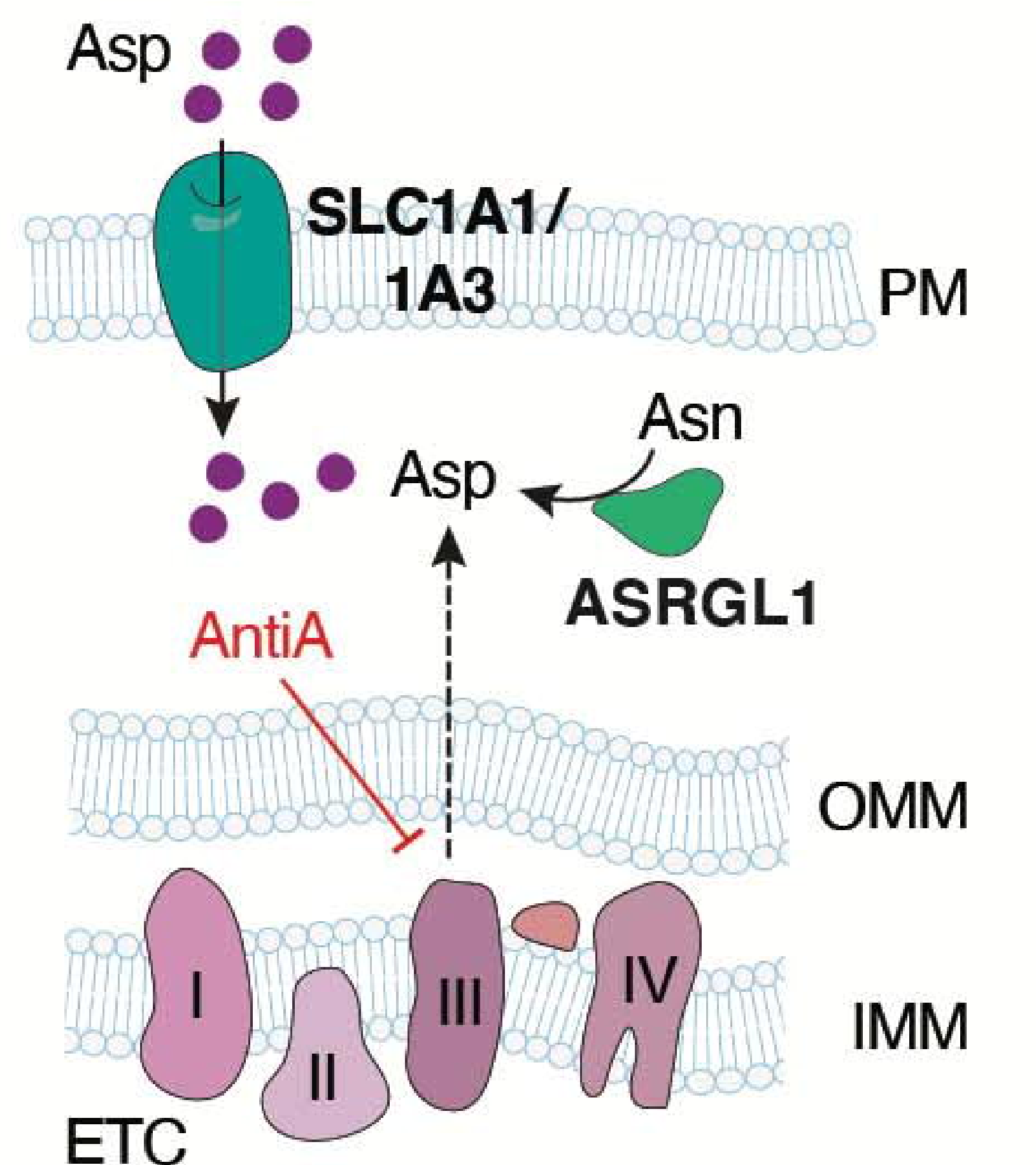

C

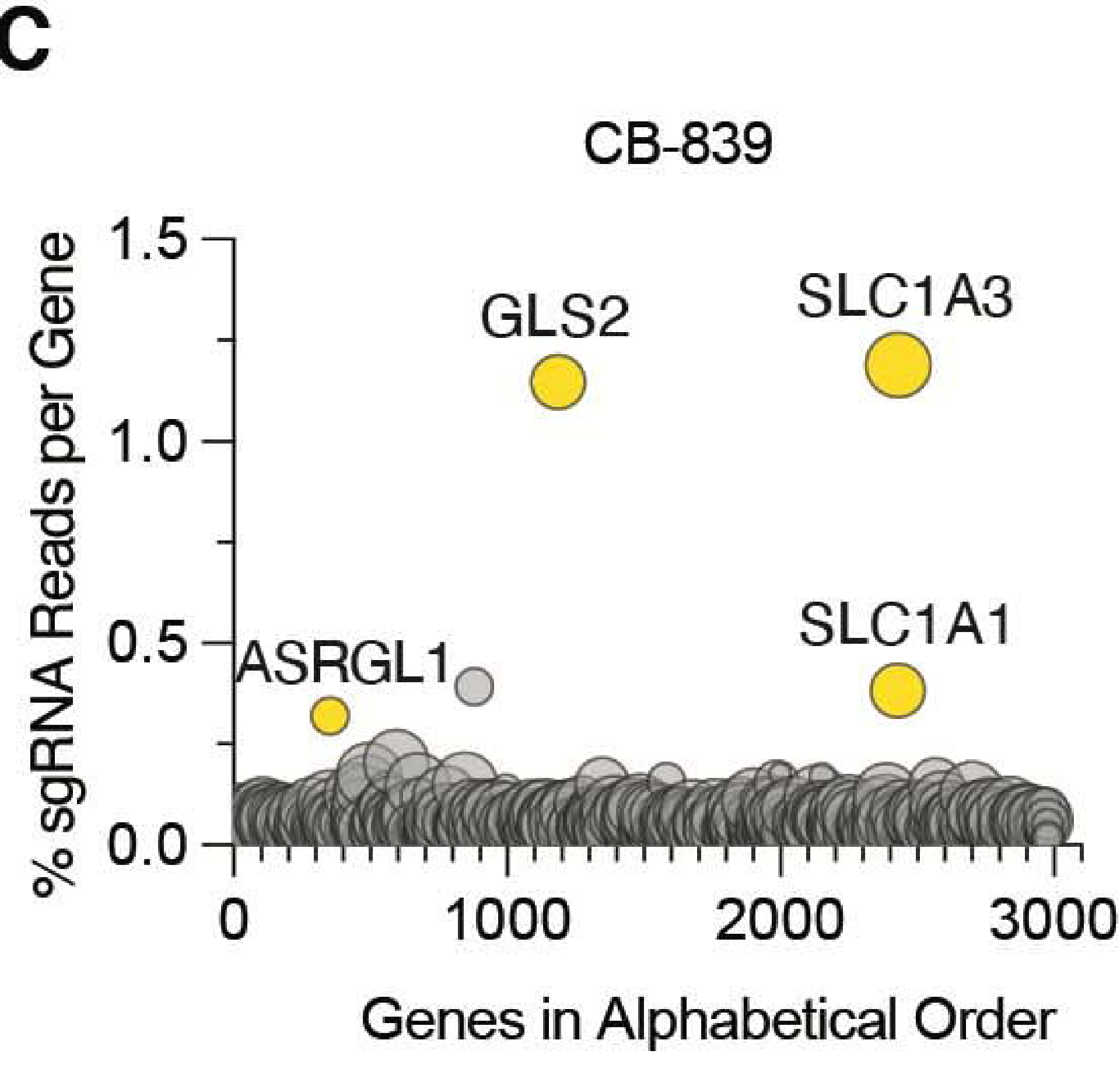

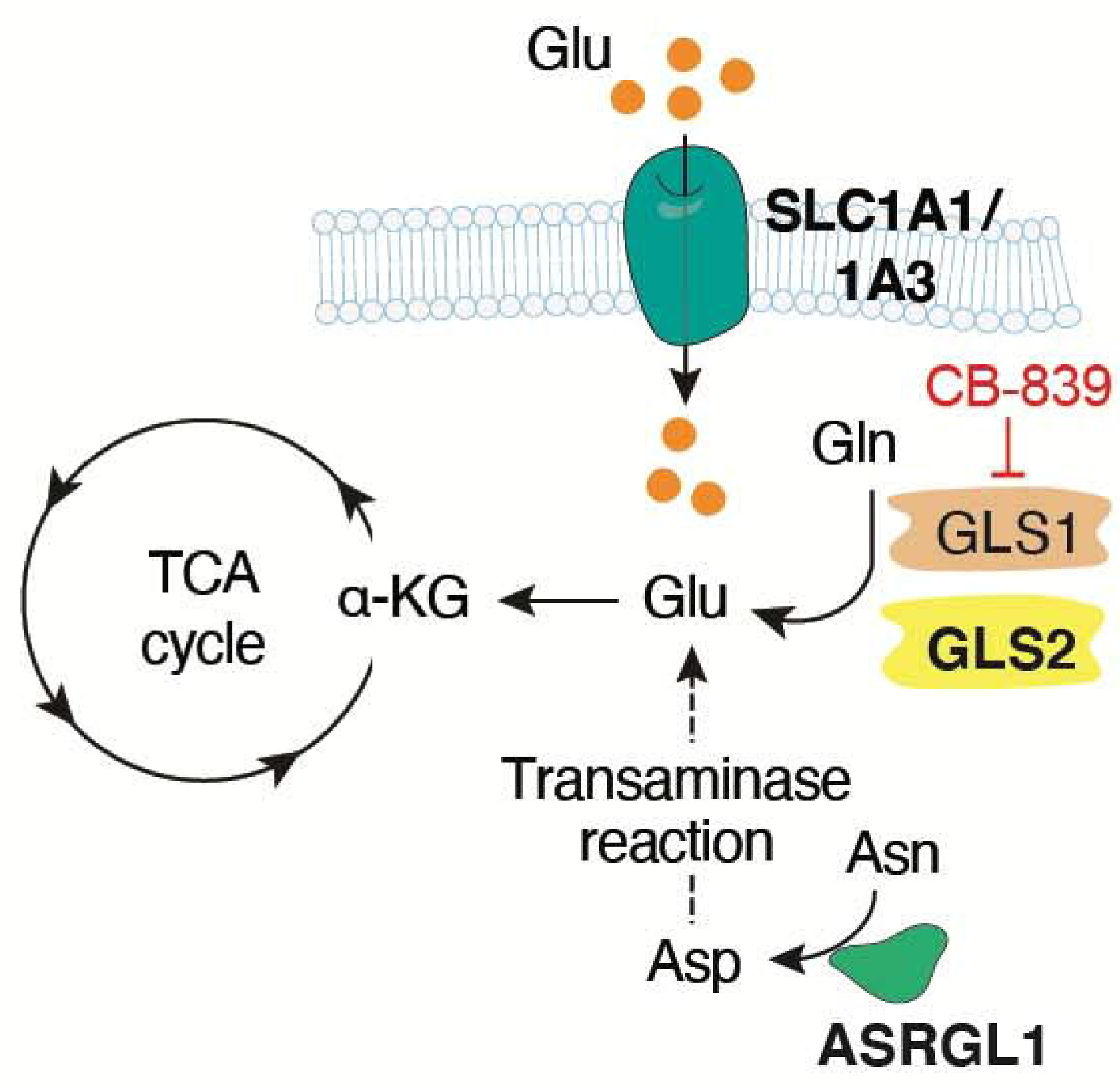

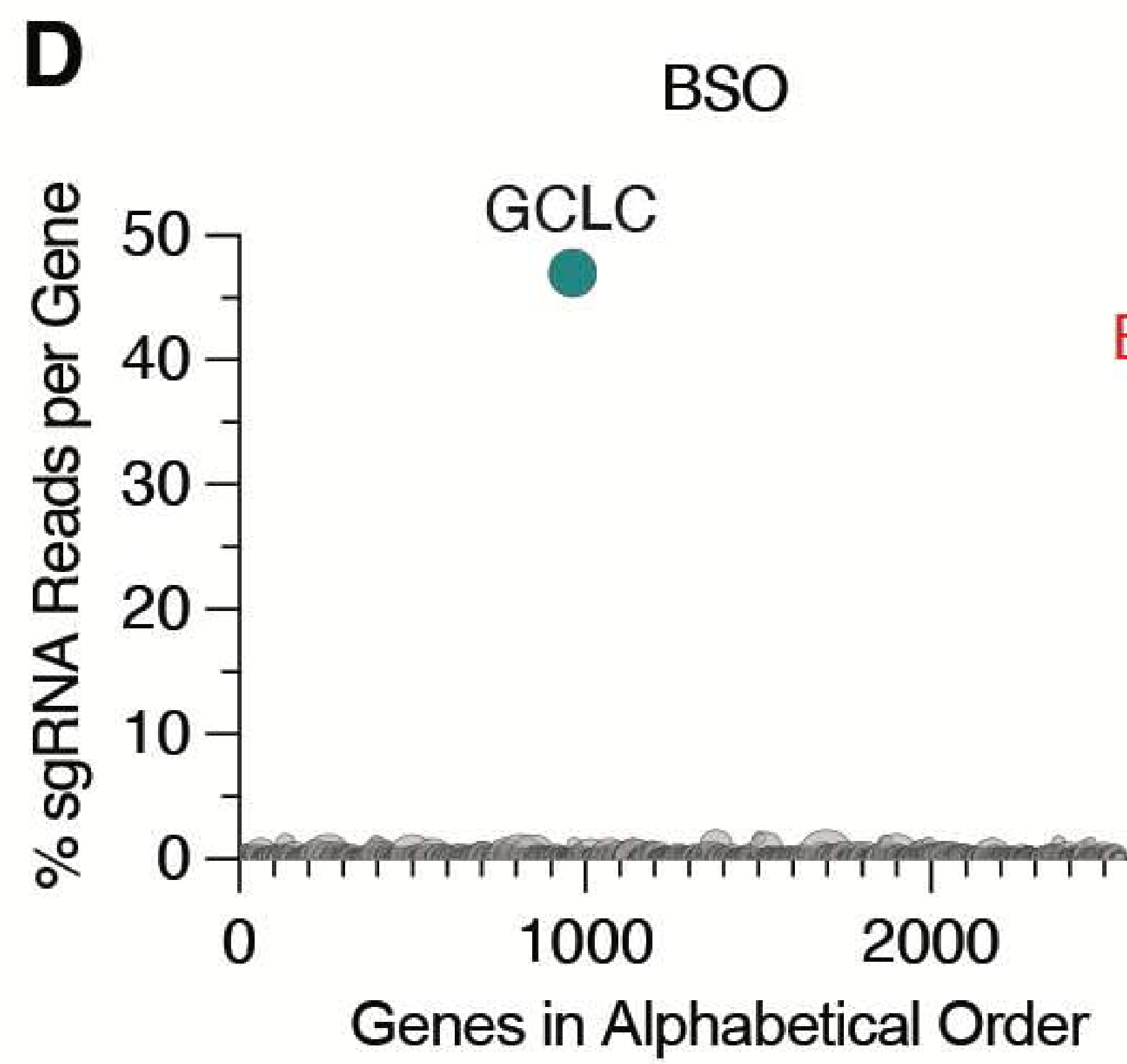

$\mathbf{F}$

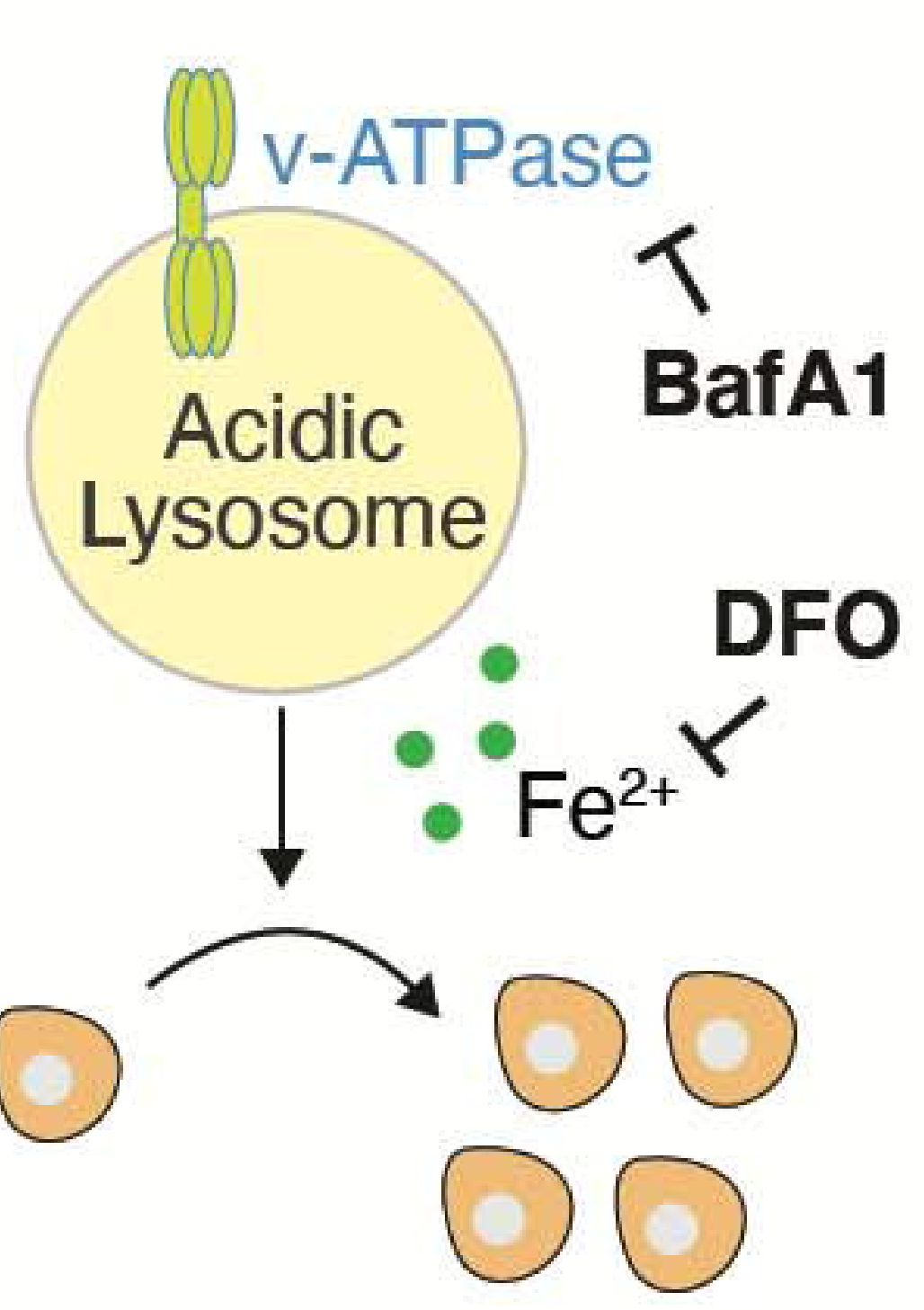

G
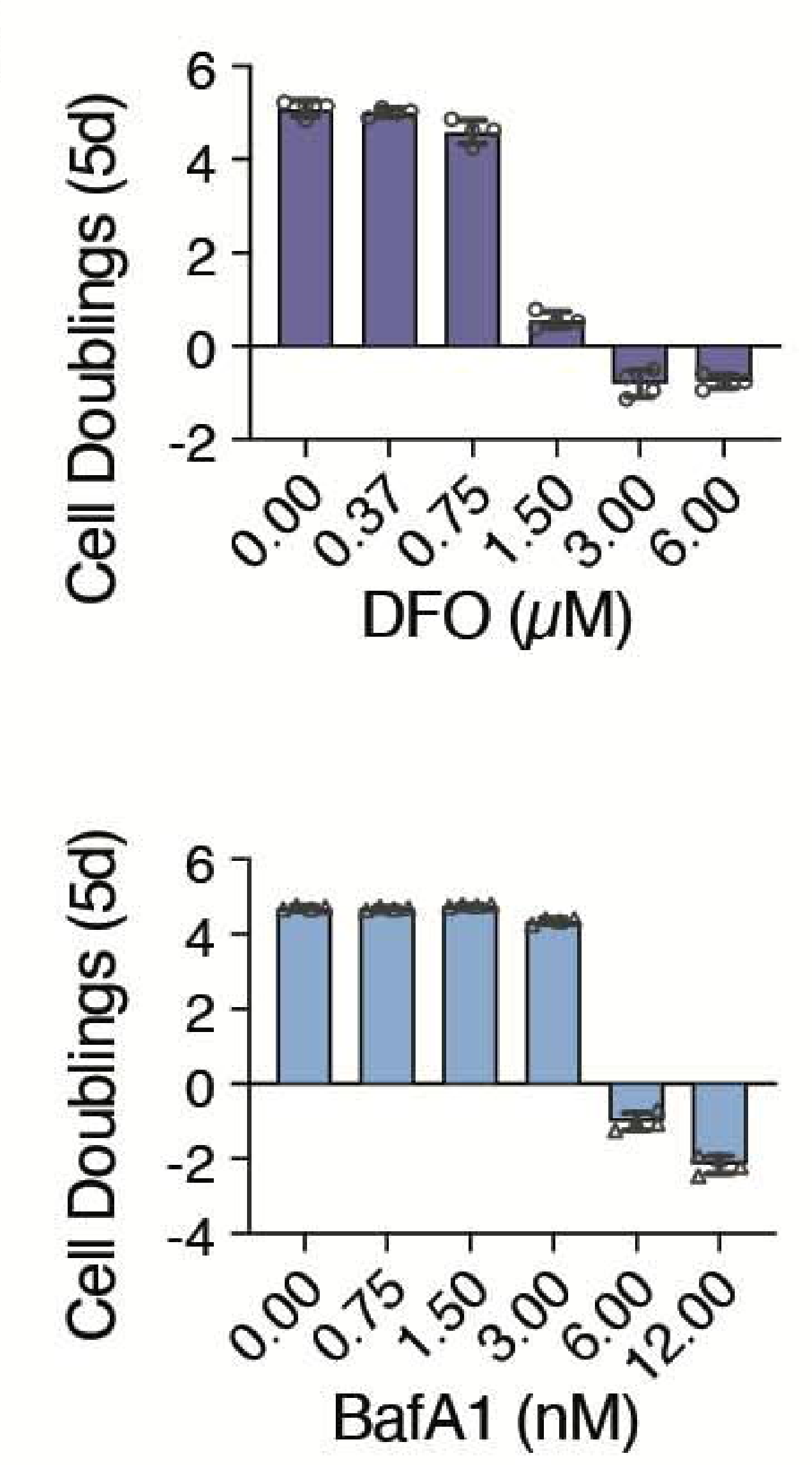

$\mathbf{J}$

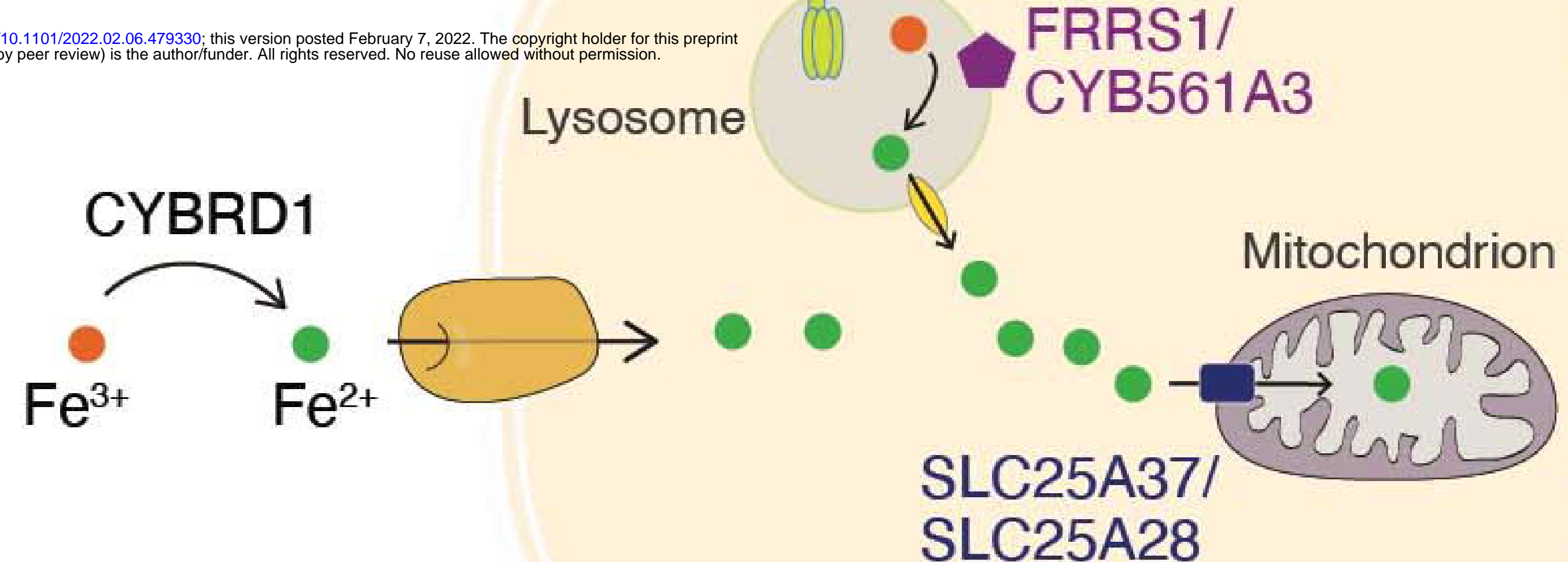

E

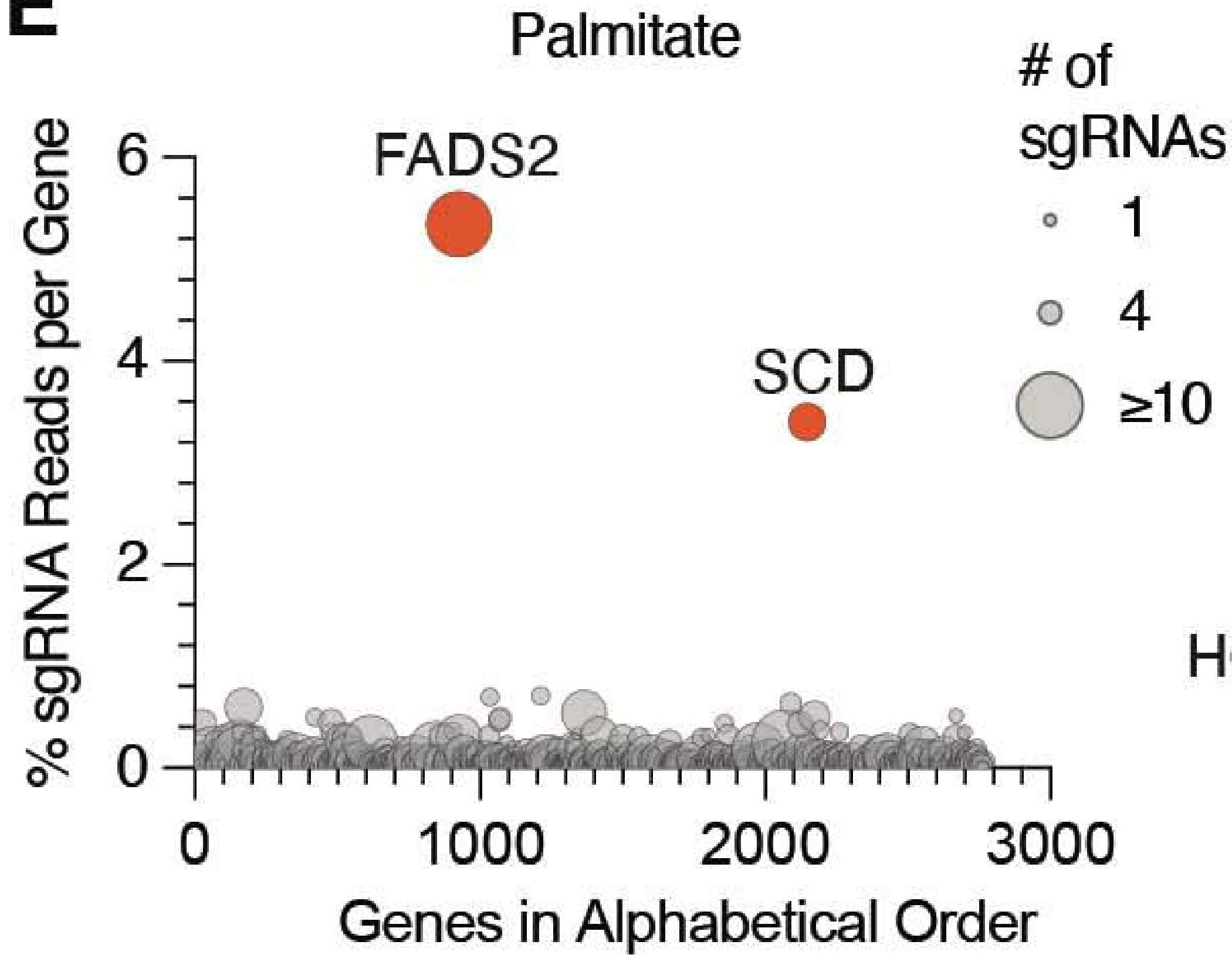

membrane

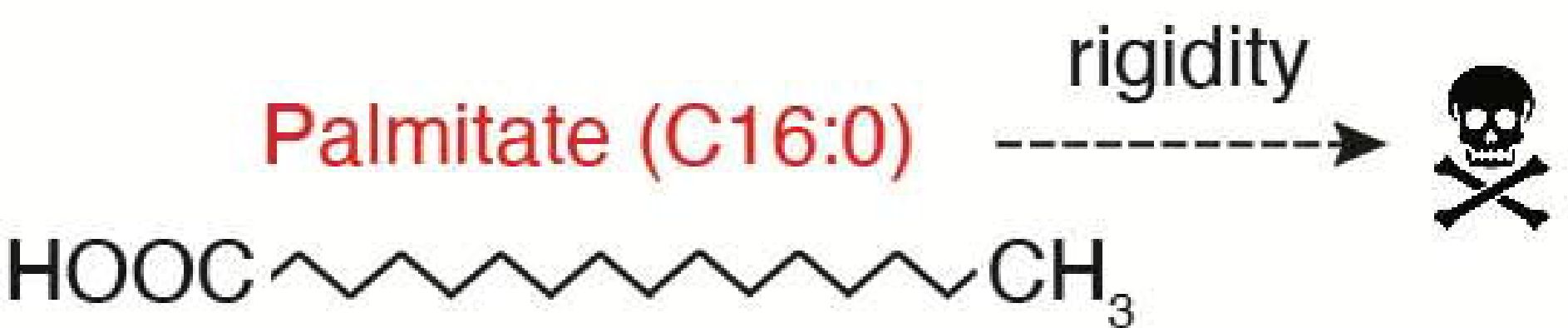

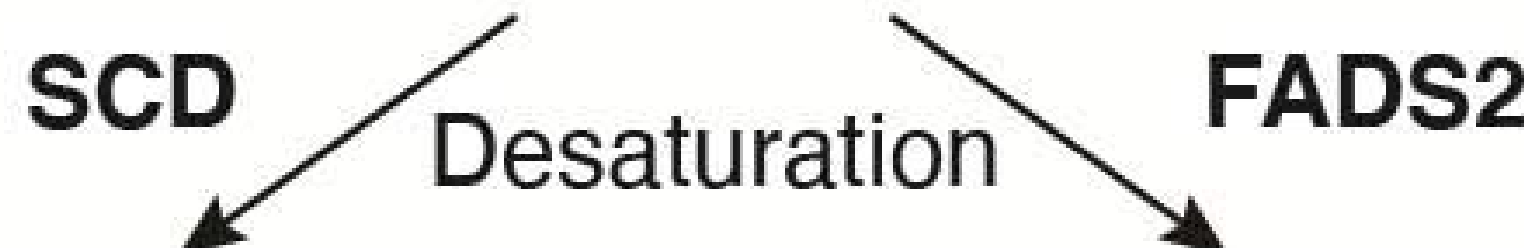

$\mathrm{HOOC} \sim \mathrm{NCH}_{3} \mathrm{HOOC}_{\mathrm{H}}^{\sim} \mathrm{NCH}_{3}$ C16:1n-7

C16:1n-10
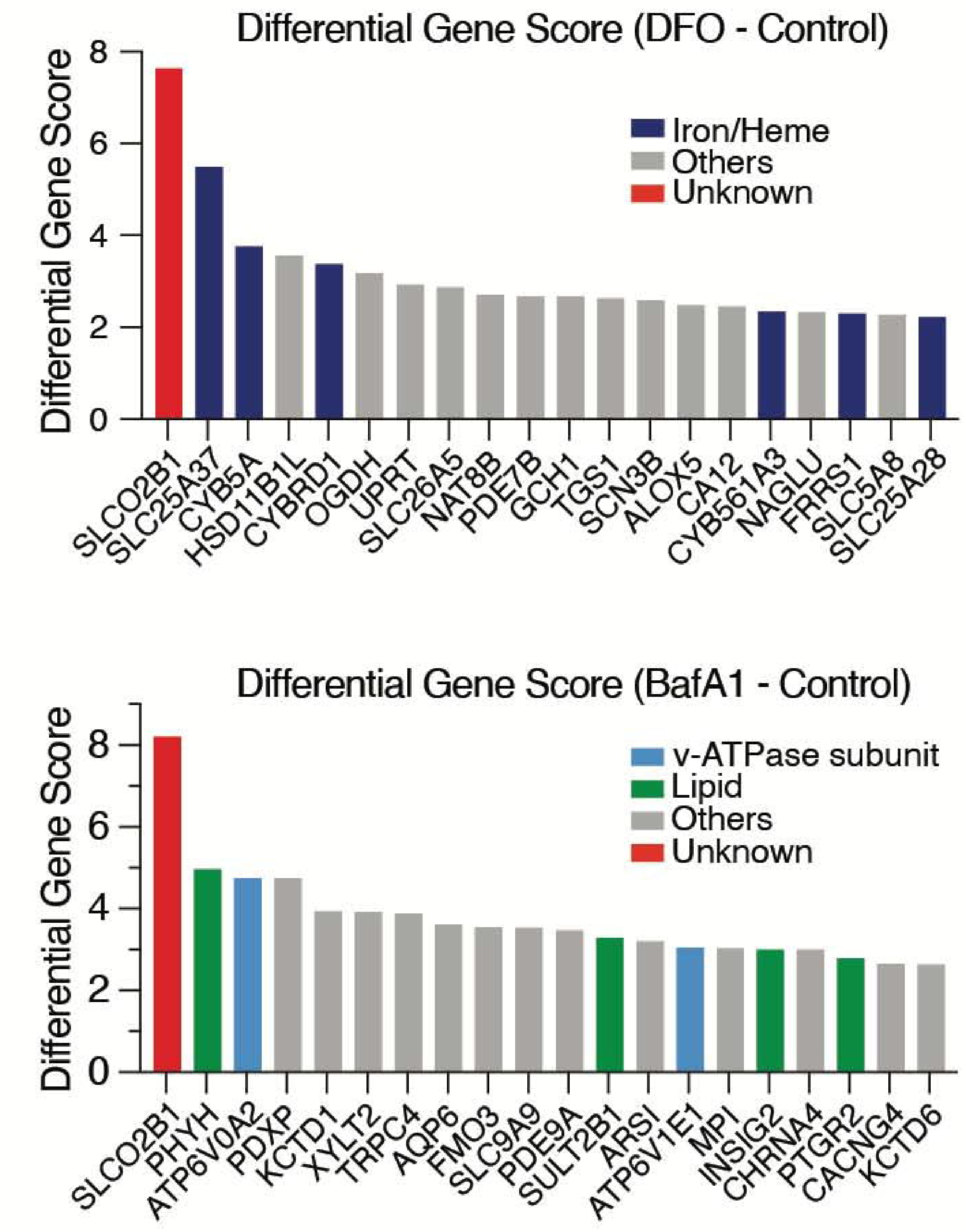

$\mathbf{K}$
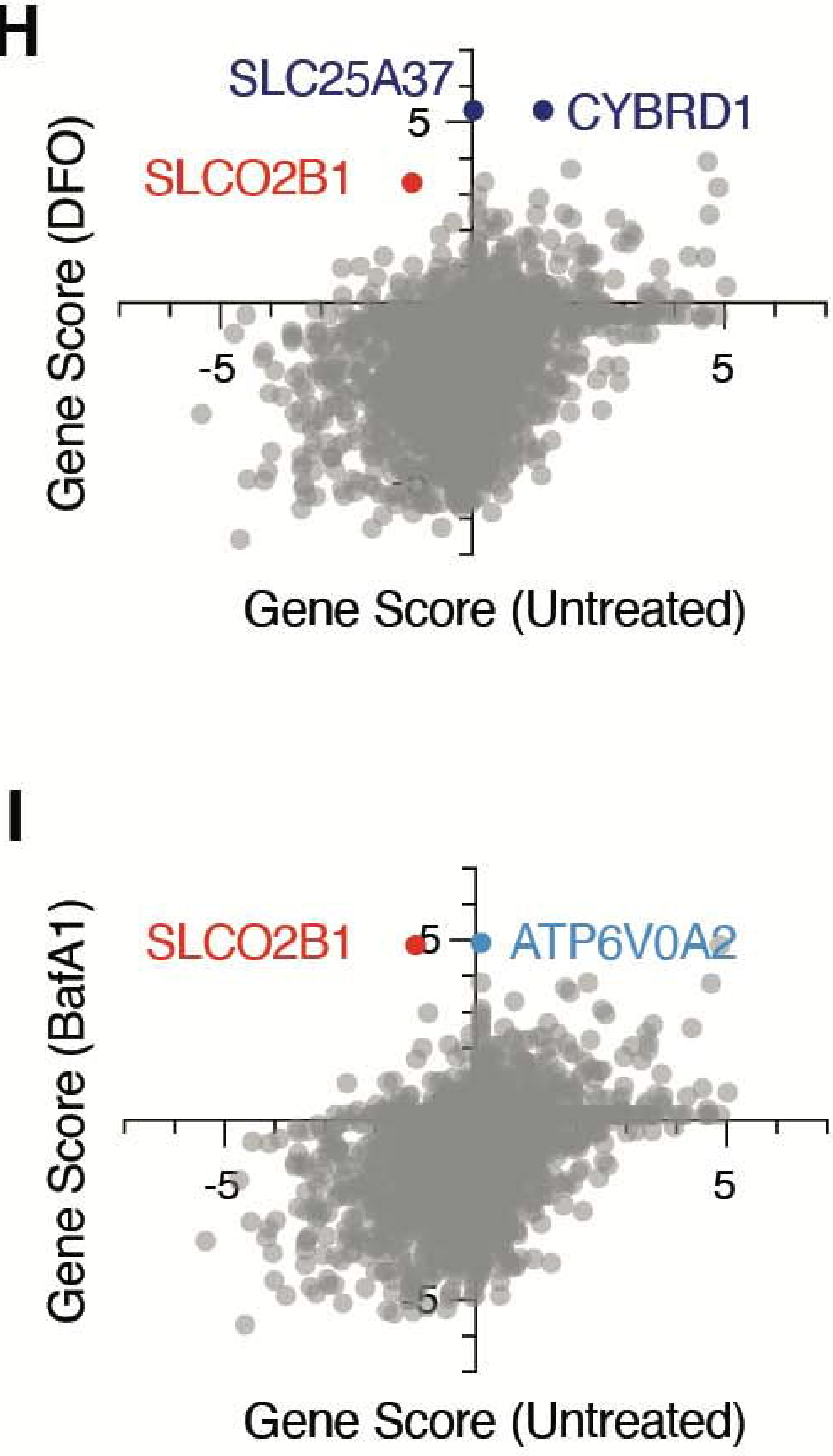

L $\rightarrow$ Vector $\rightarrow$ SLC25A37

DFO $(3 \mu \mathrm{M})$

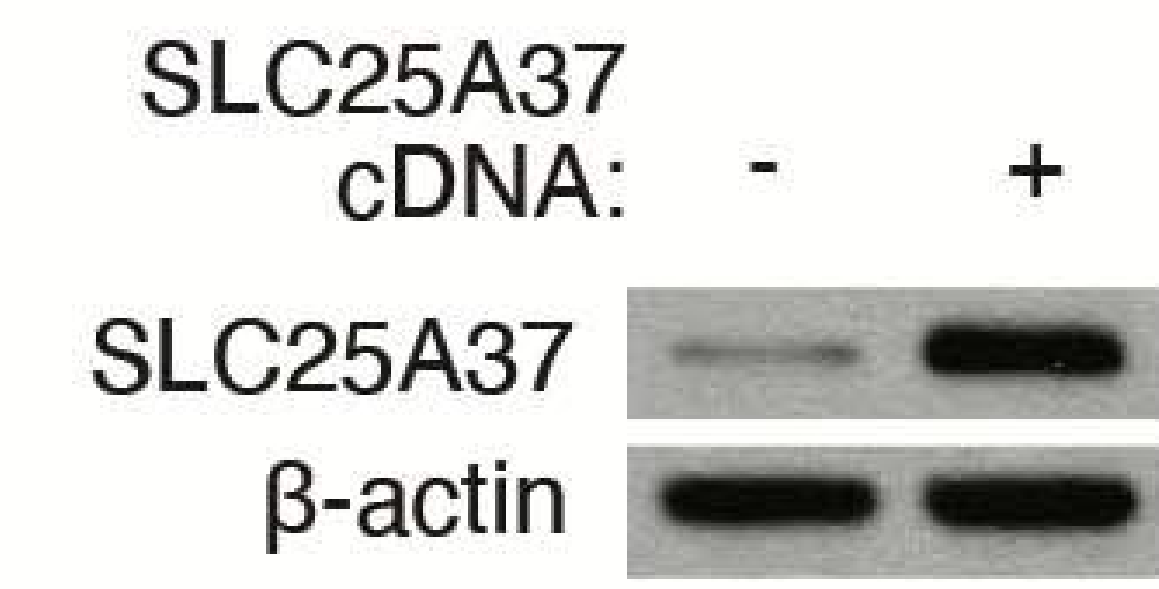

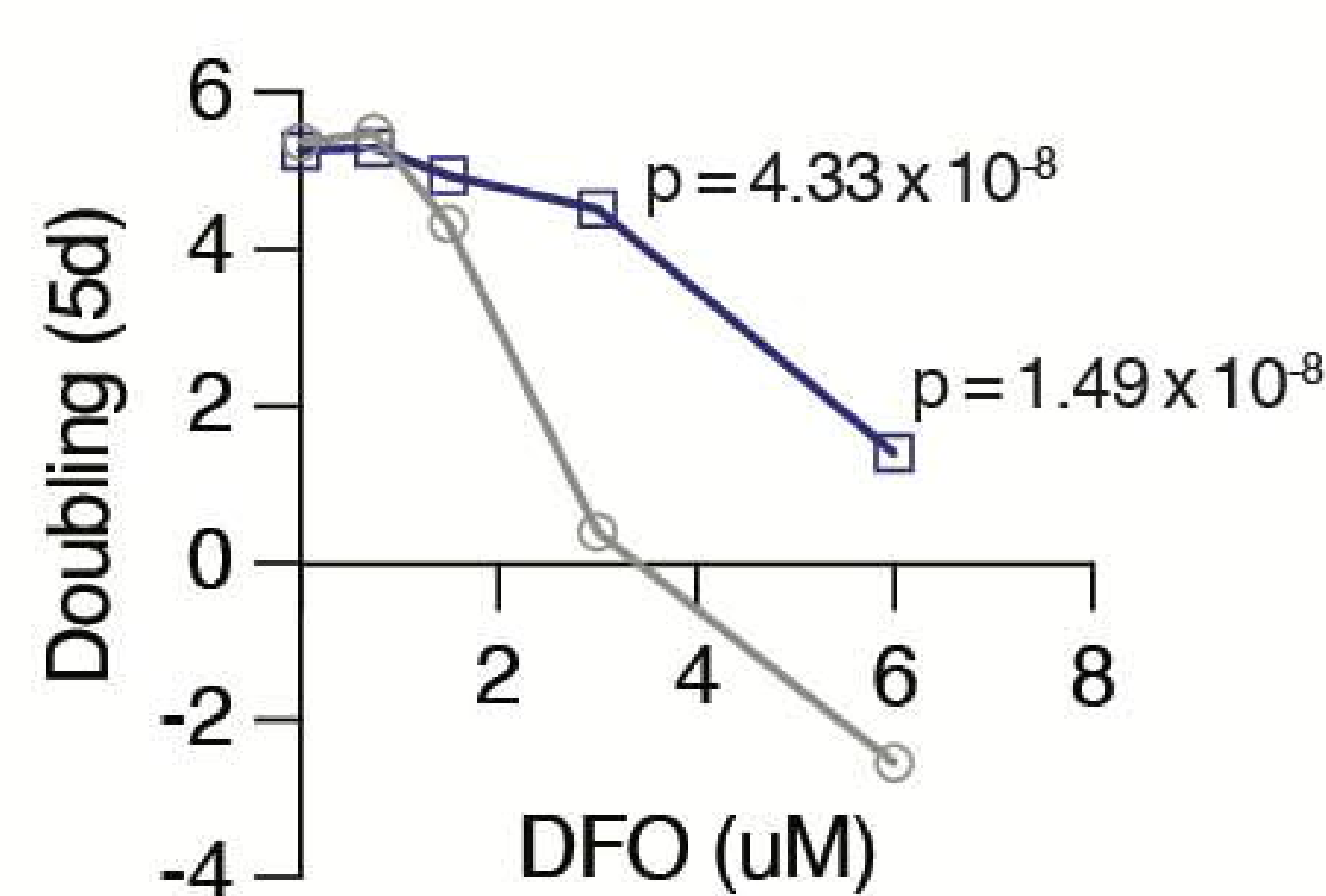

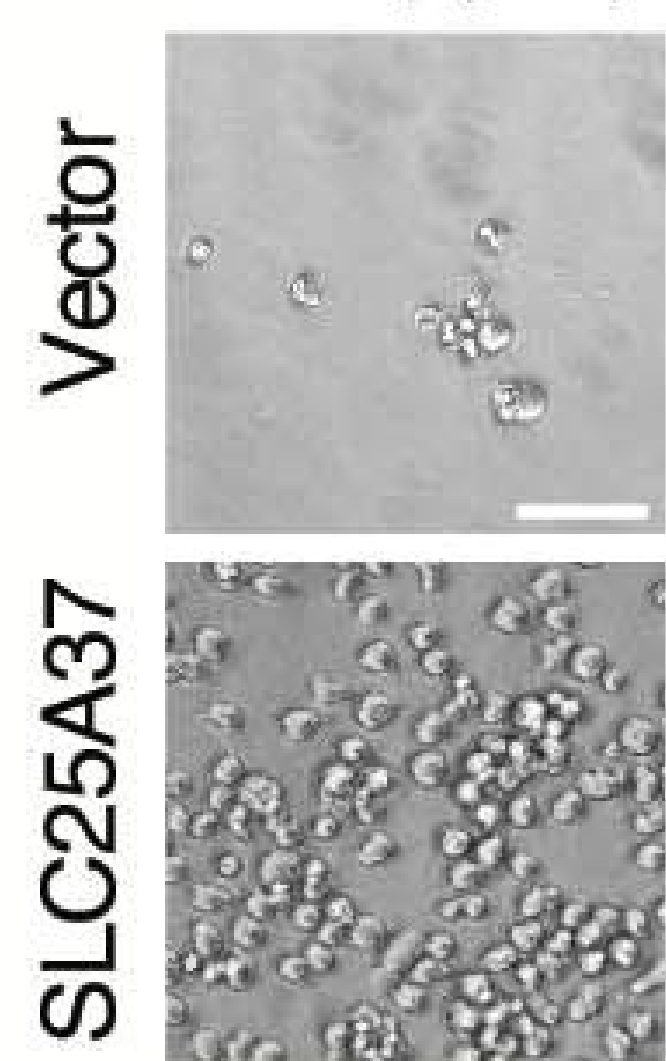


A

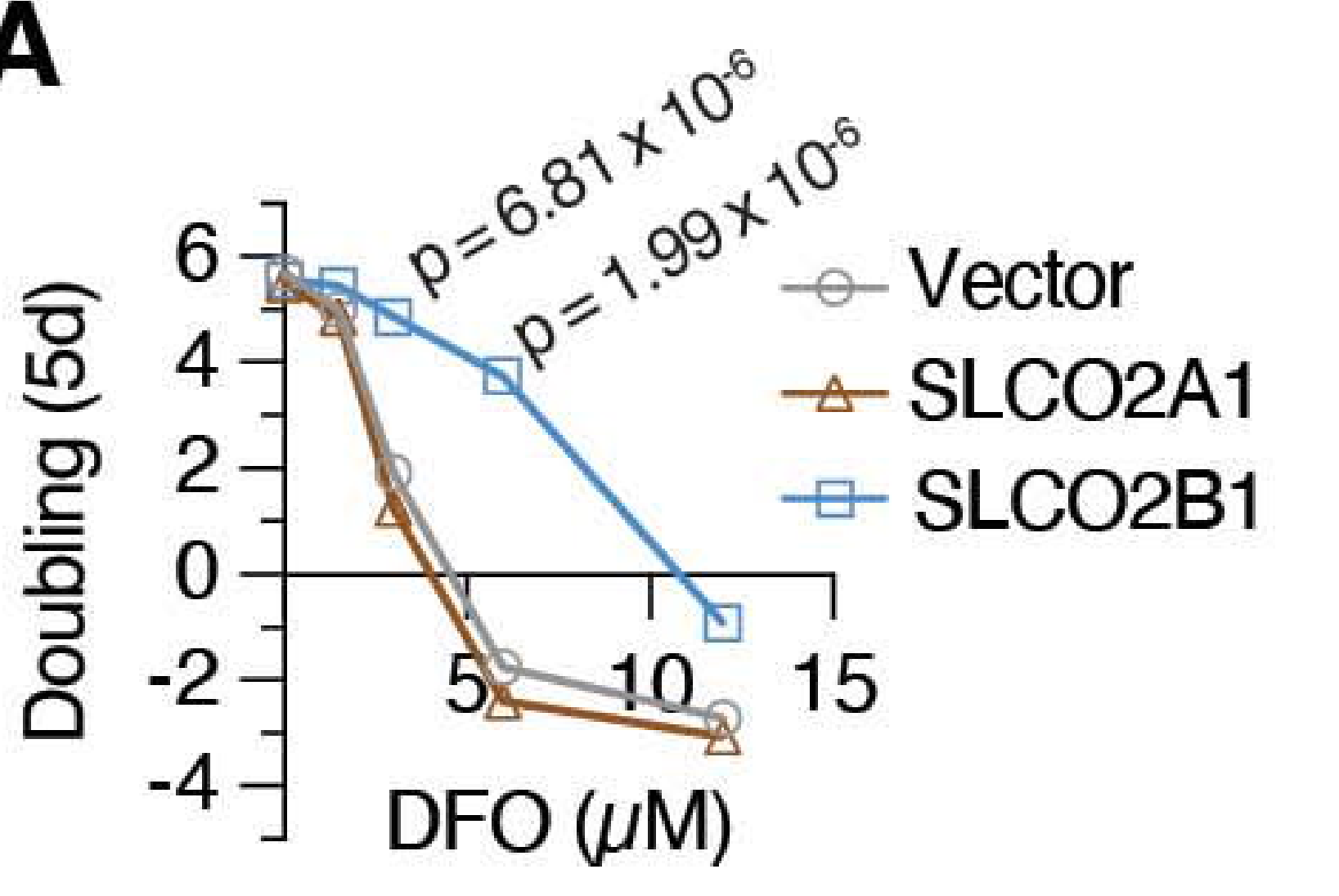

C

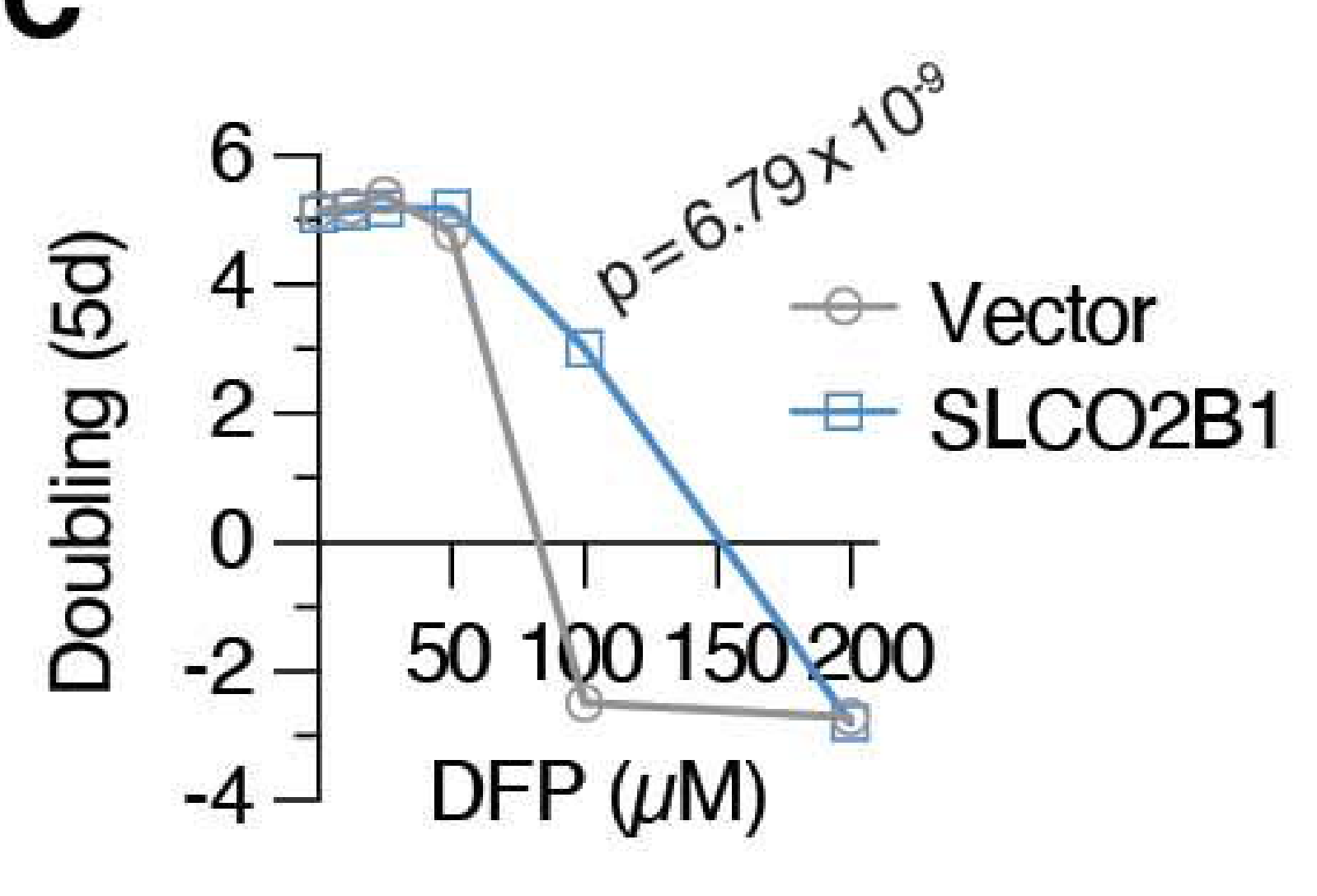

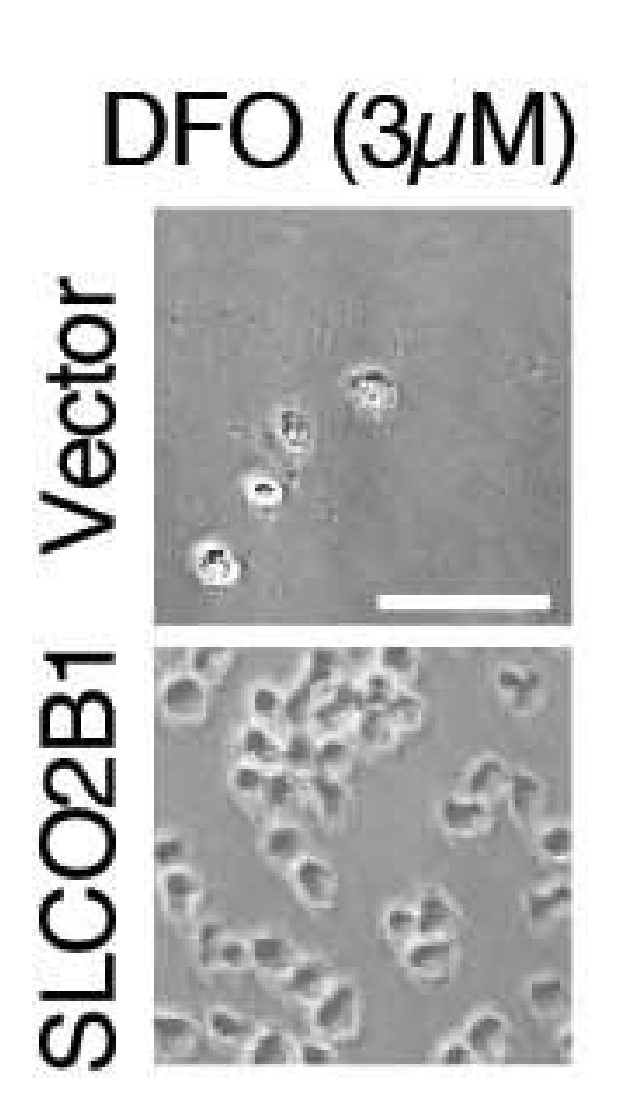

B
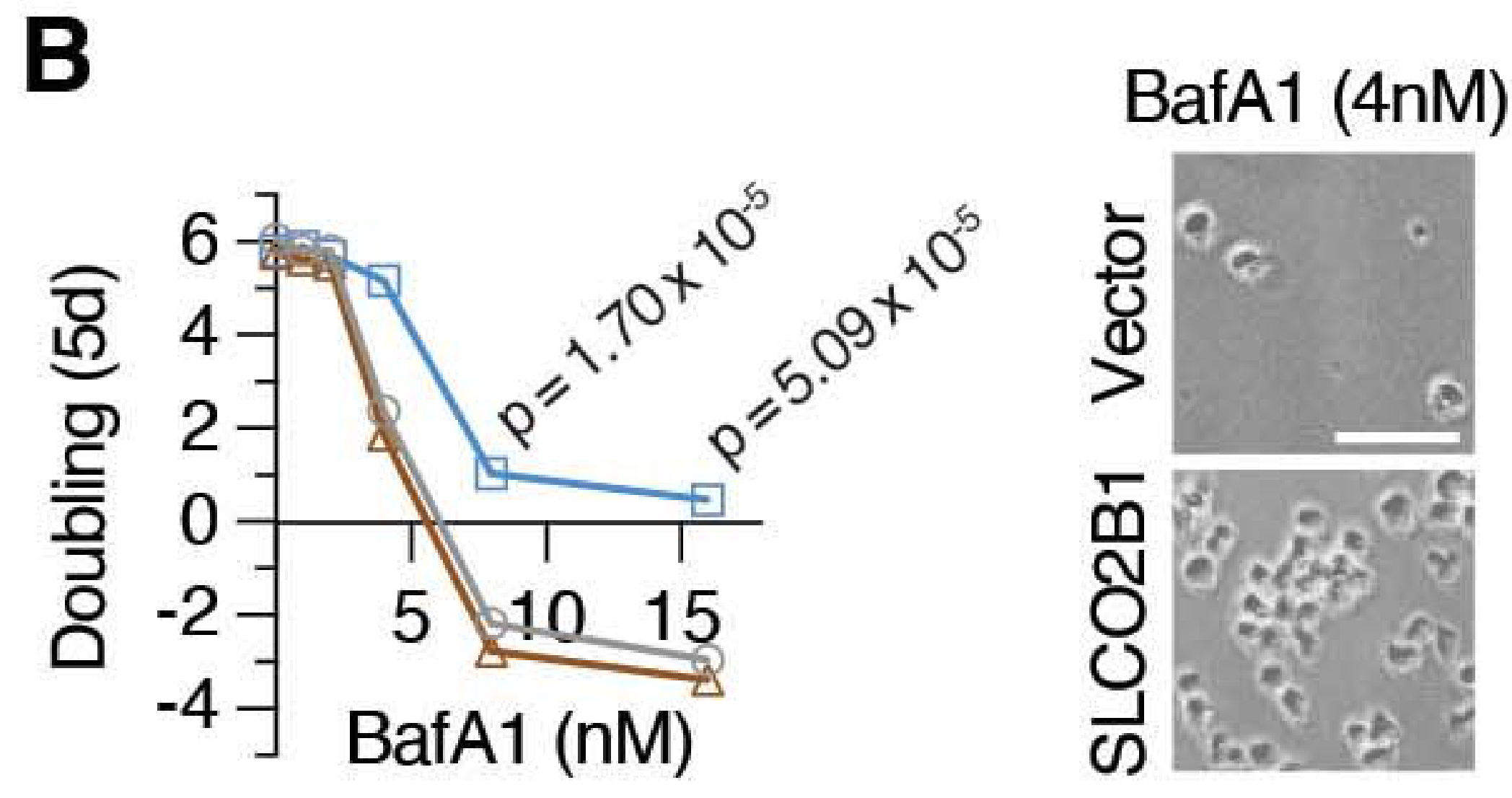

$\mathbf{F}$

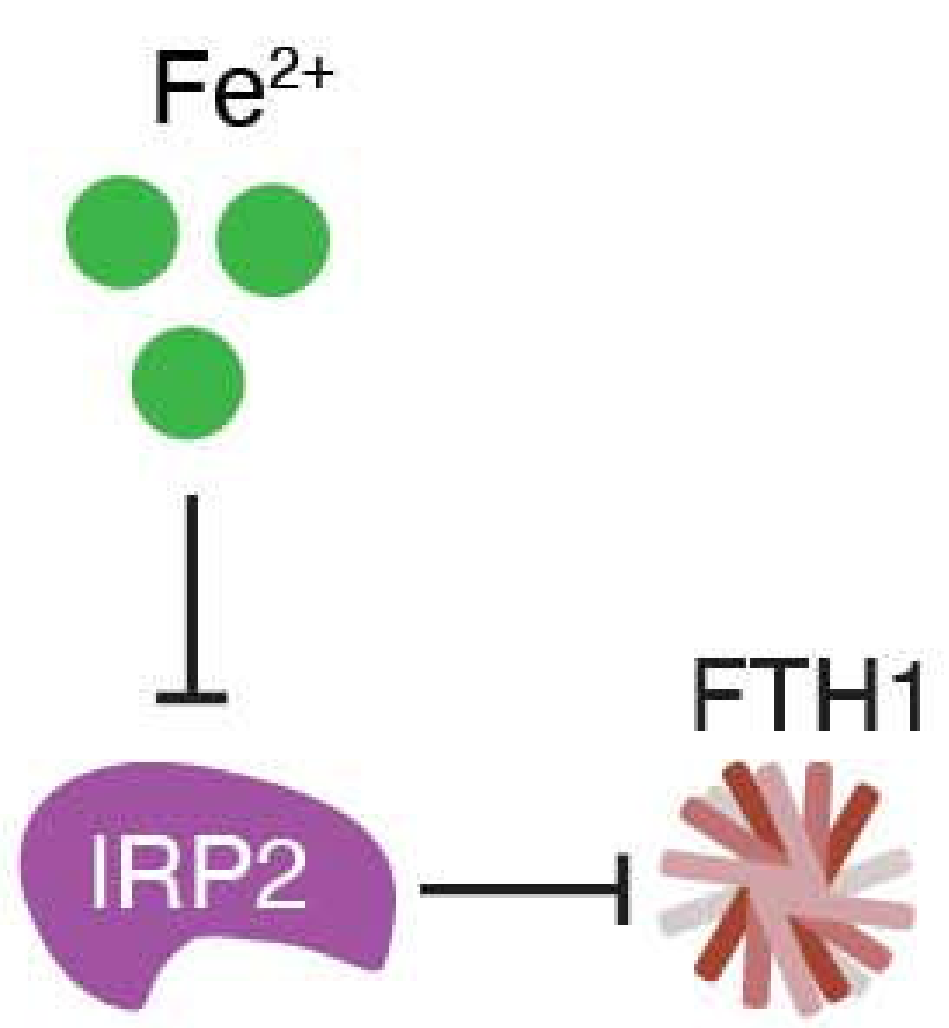

Human lines Mouse lines
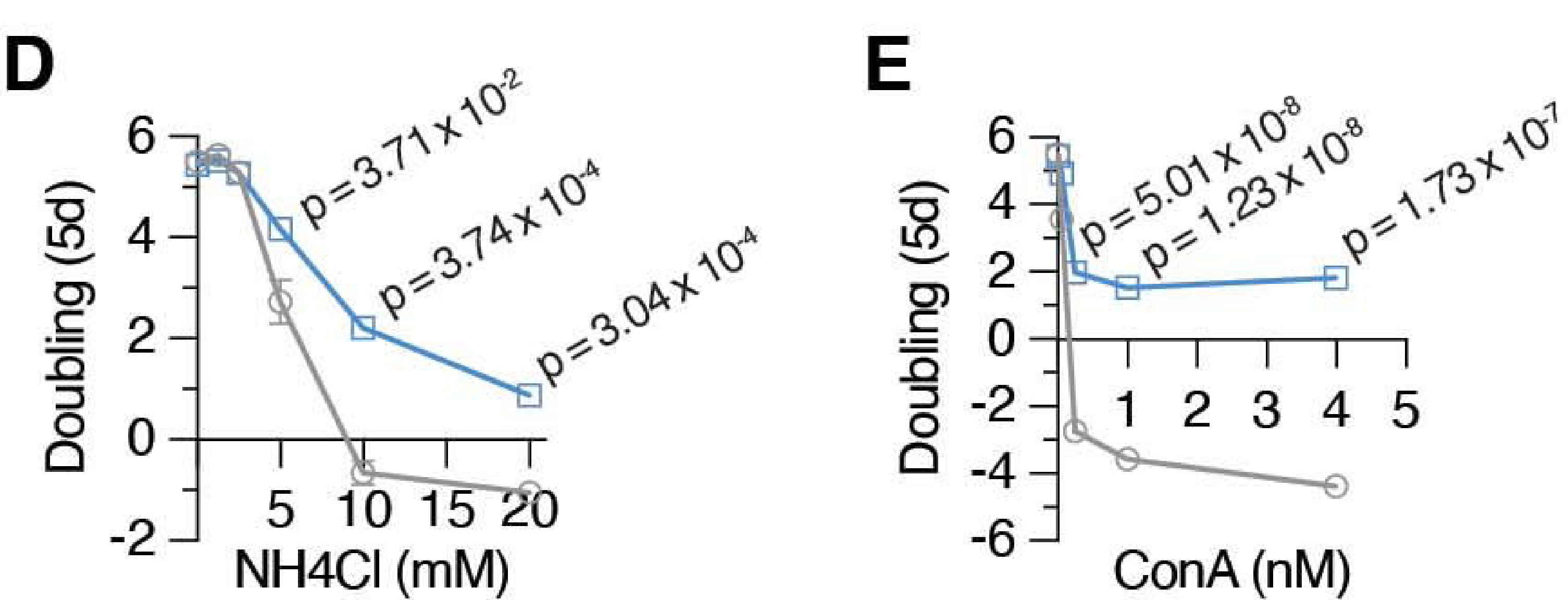

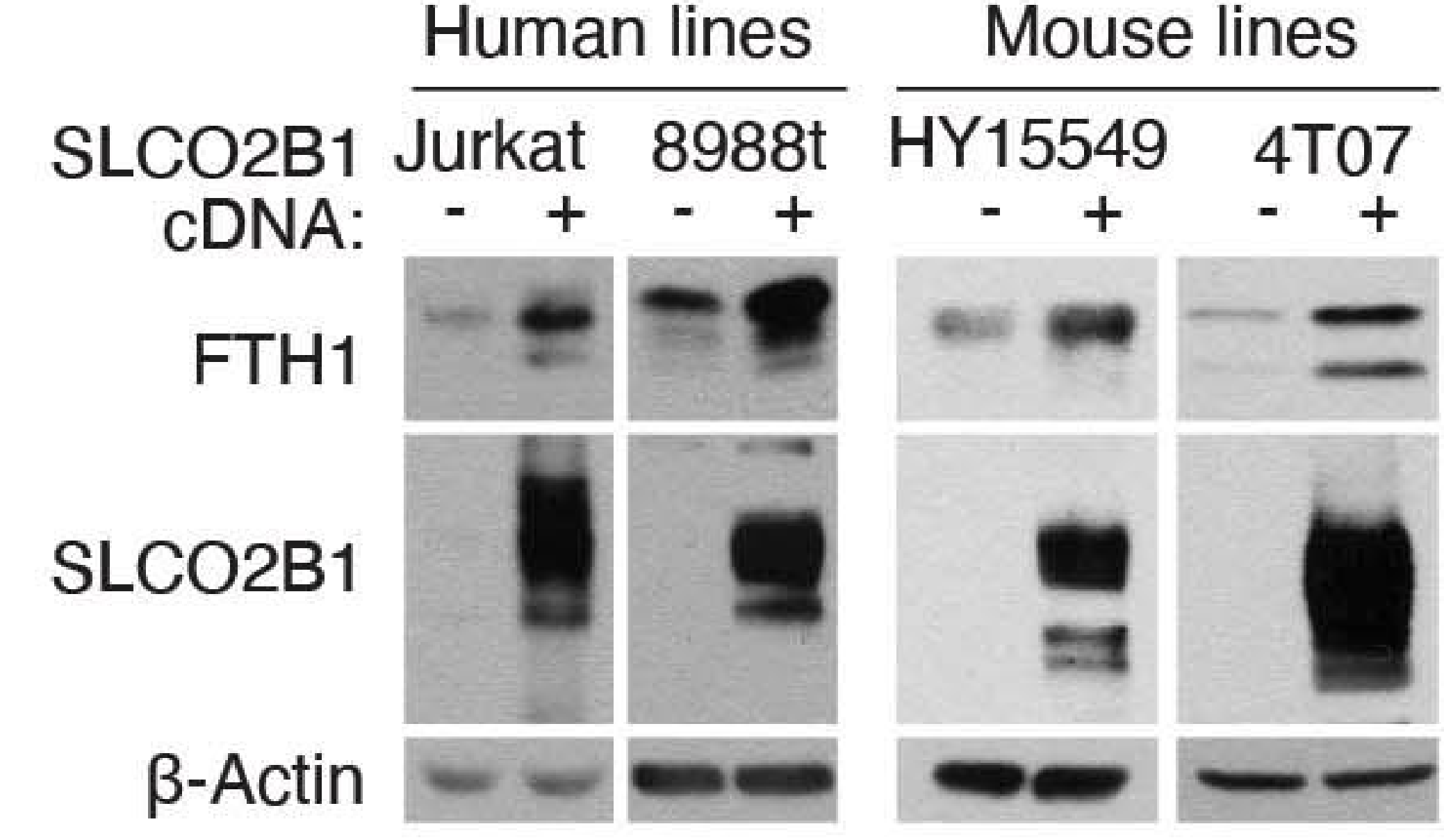

J $\circ$ Vector $\triangle$ SLCO2A1 $\square$ SLCO2B1

I

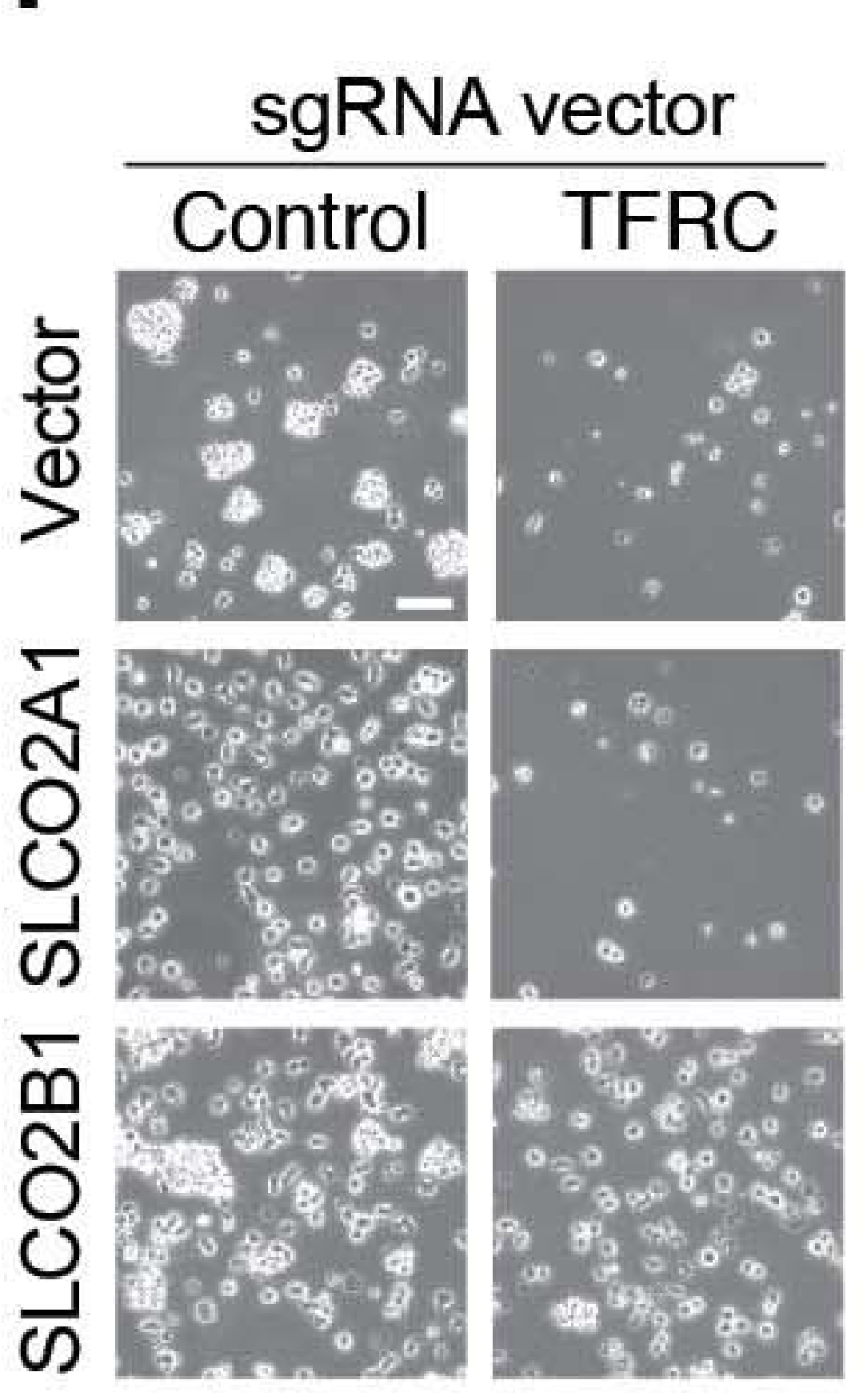

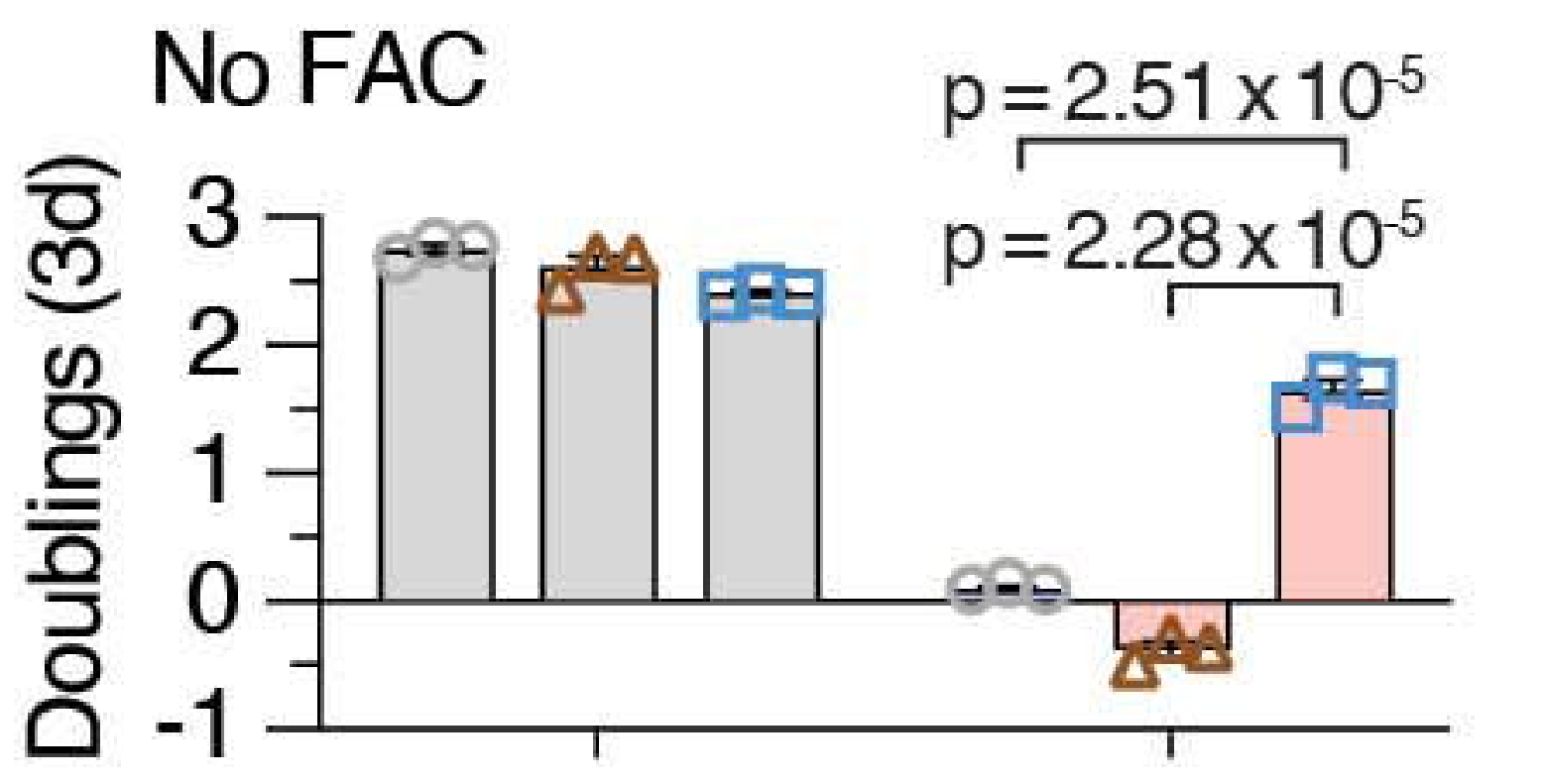

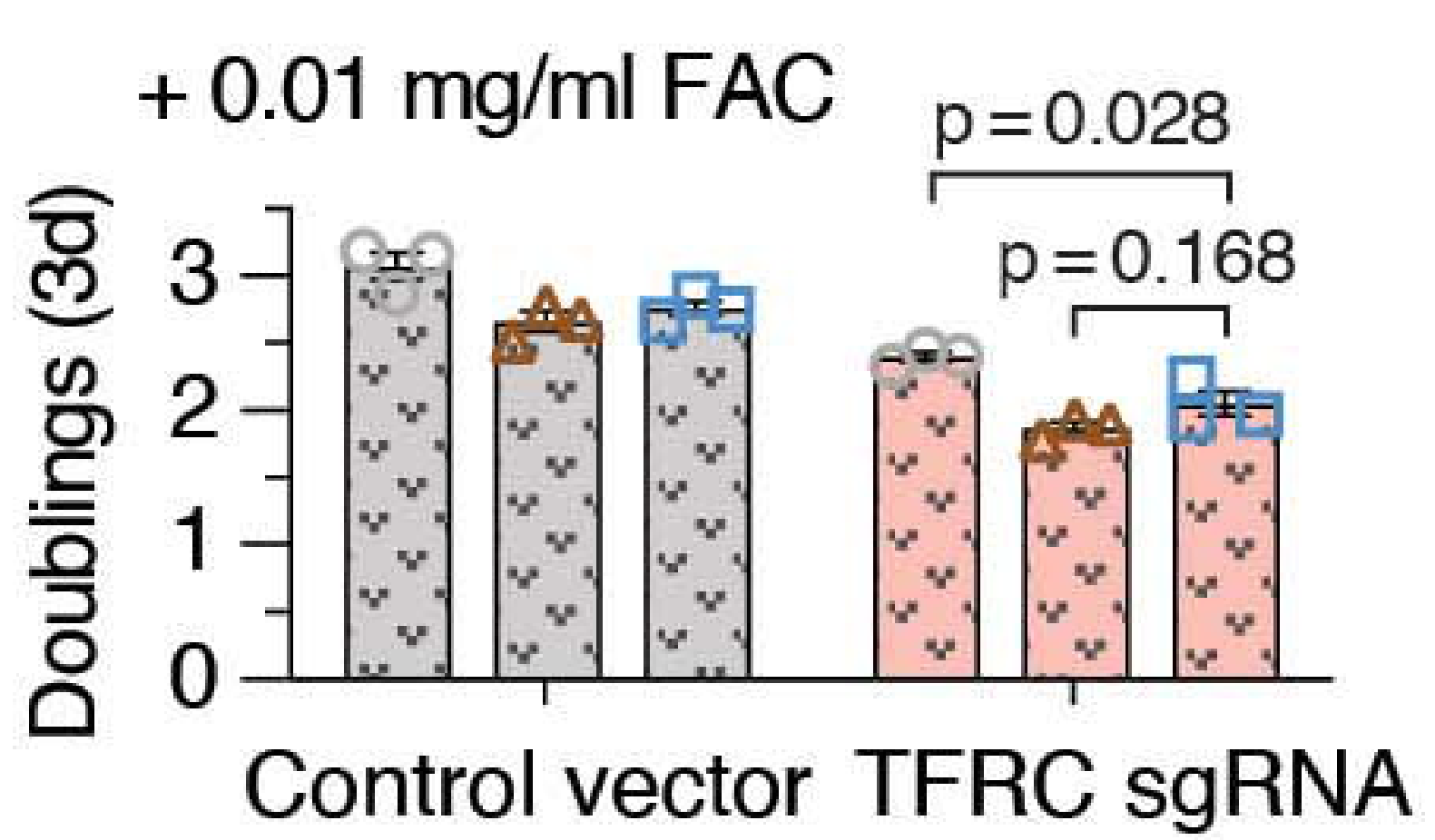


A

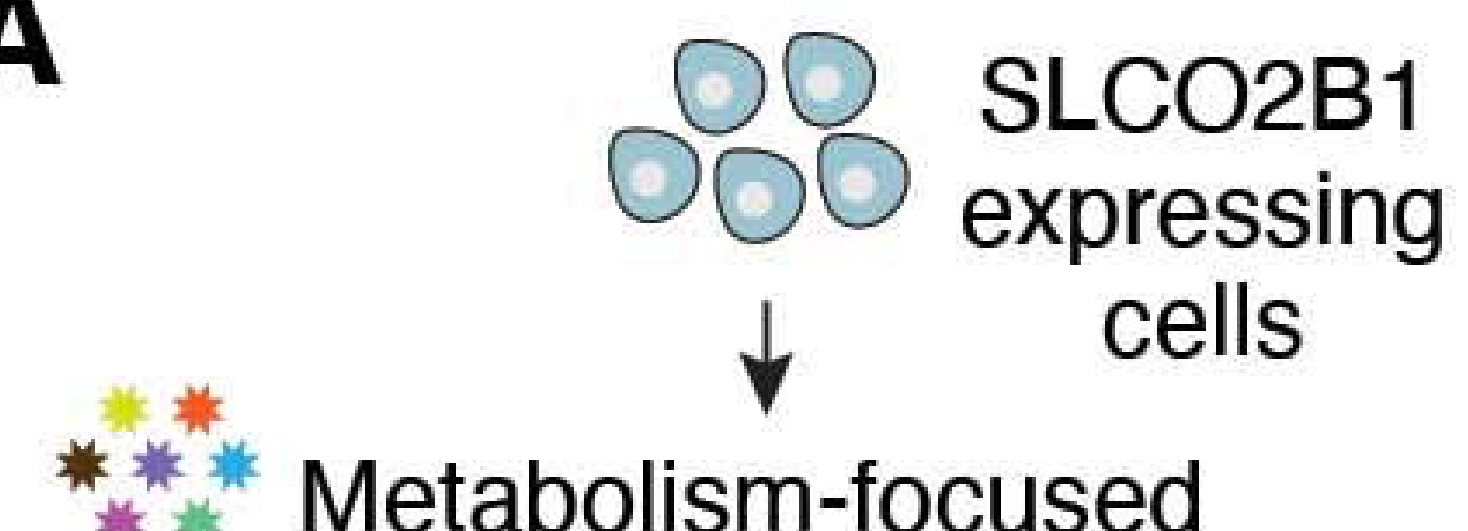
sgRNA library (23,971 sgRNAs)

$\downarrow$ infection $\downarrow$

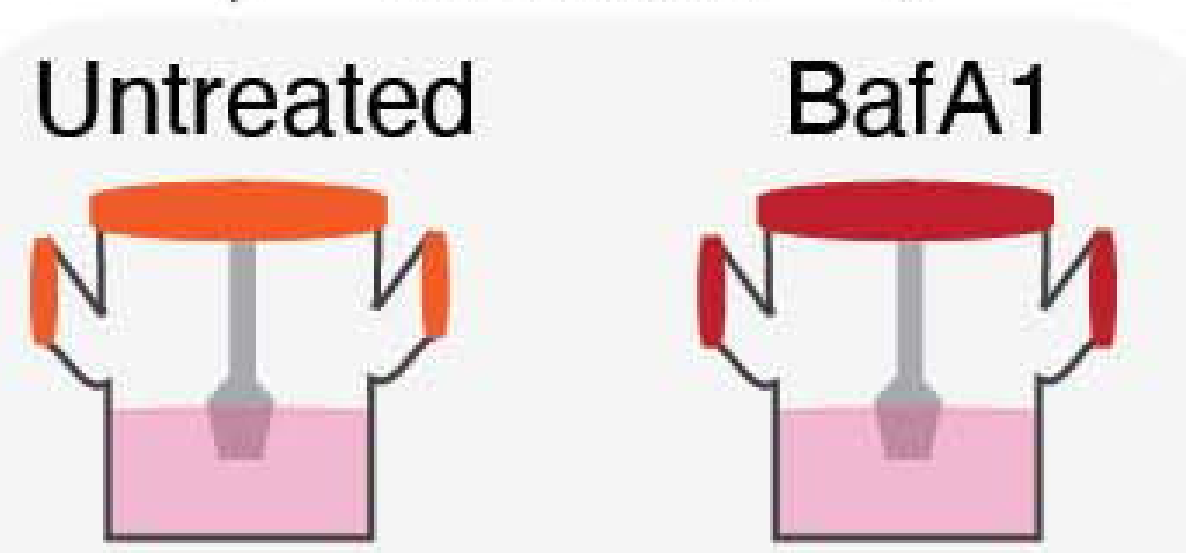

14 doublings

Next-Gen $\downarrow$ sequencing

Compare initial, untreated and BafA1-treated sgRNA abundance

D

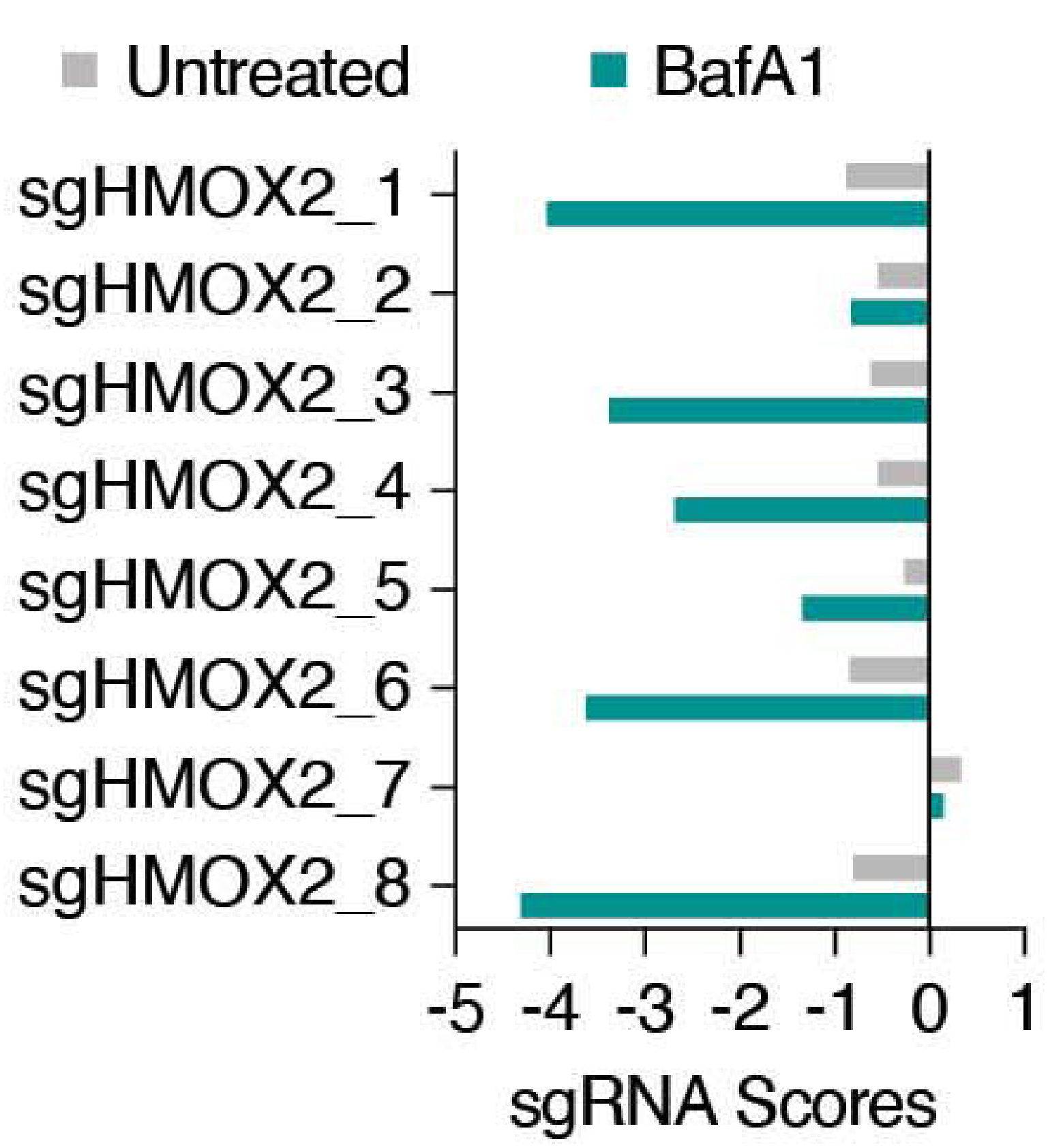

\section{H}

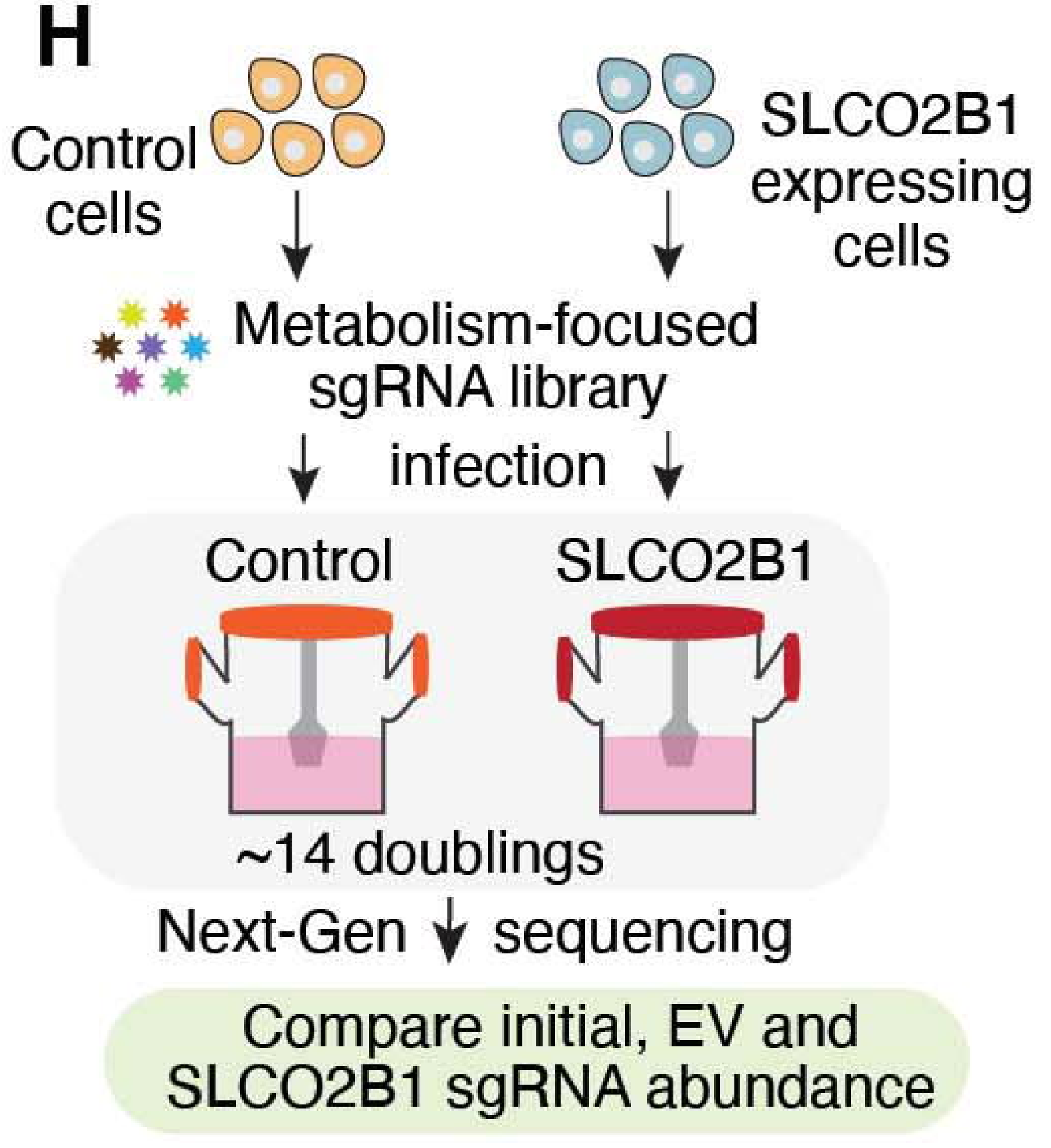

B

BafA1 vs. Untreated CRISPR screen in SLCO2B1-overexpressing cells

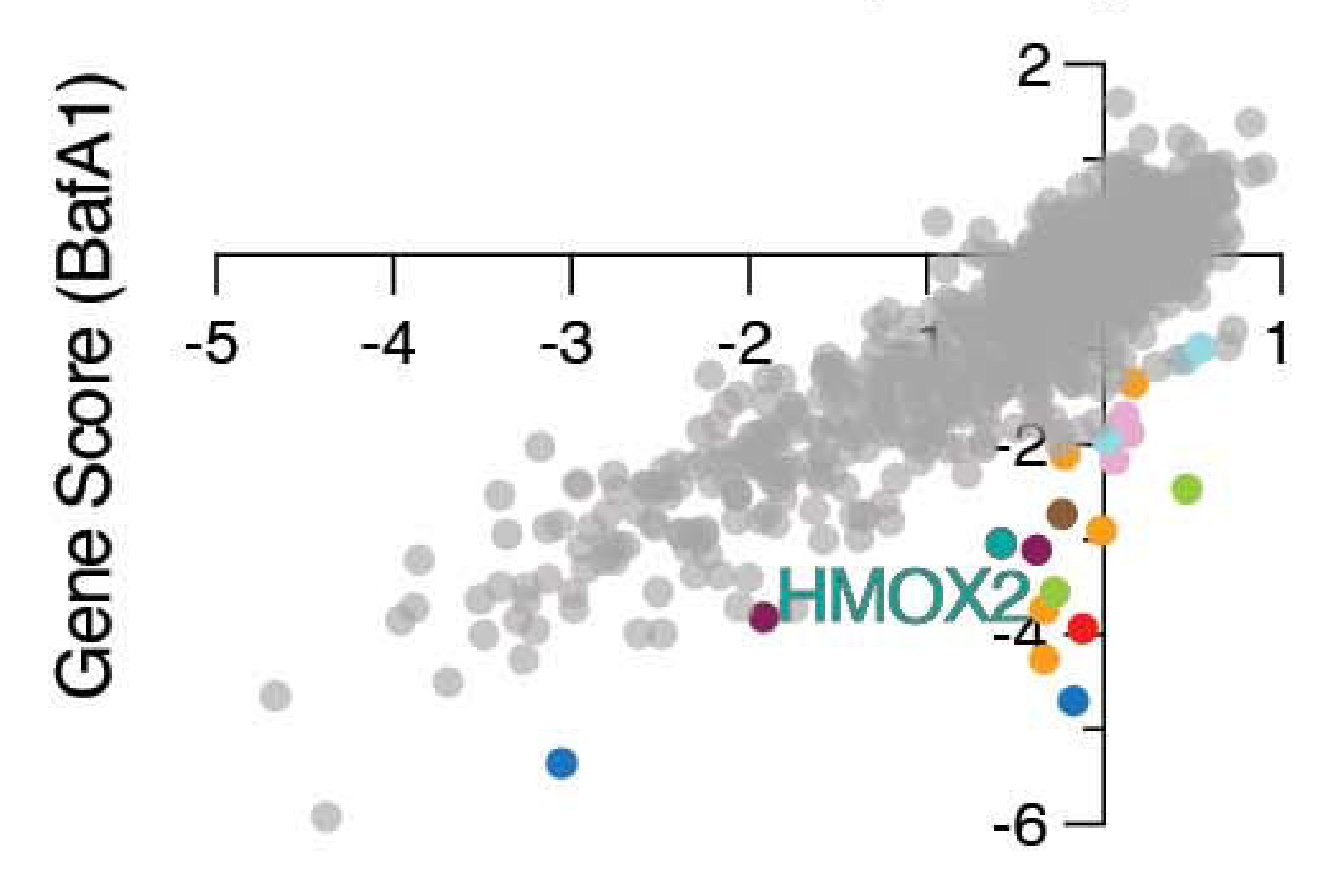

Gene Score (Untreated)

C

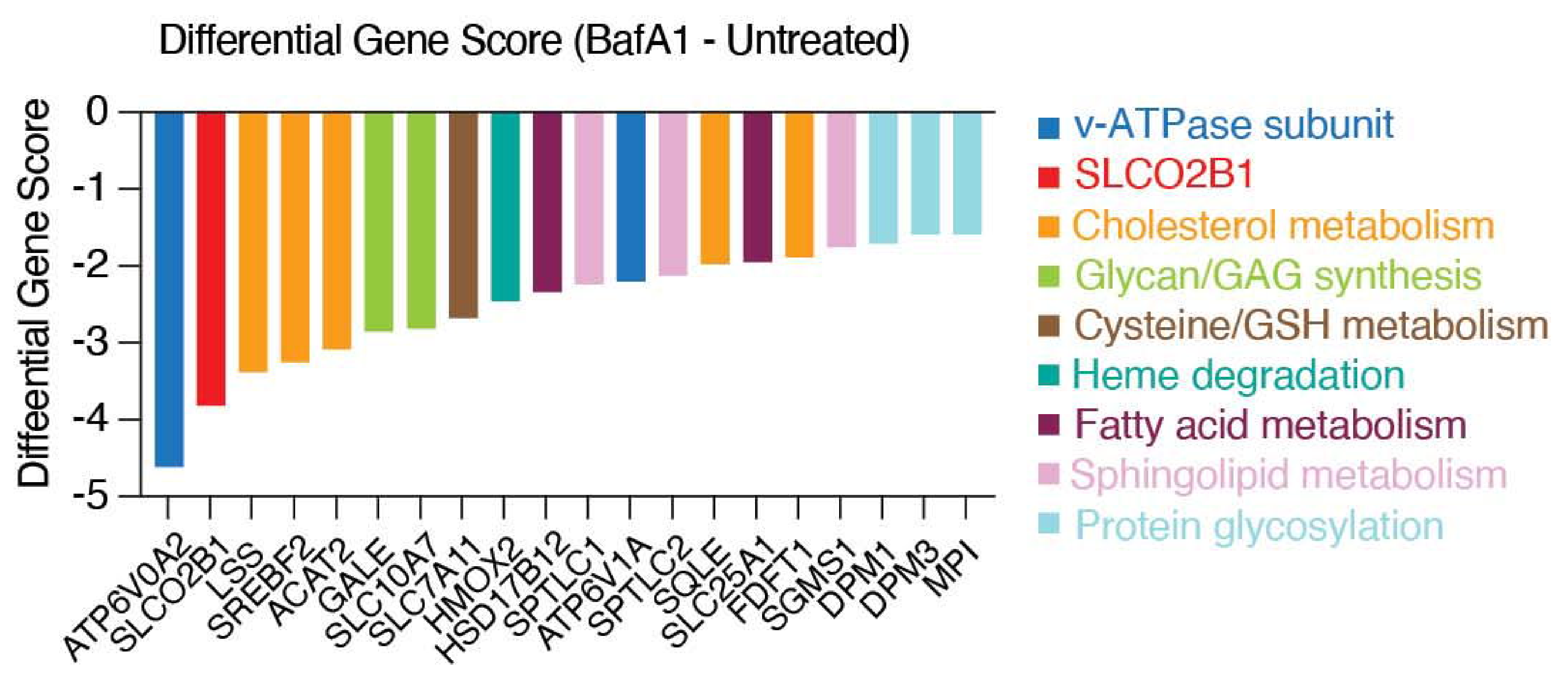

$\mathbf{E}$

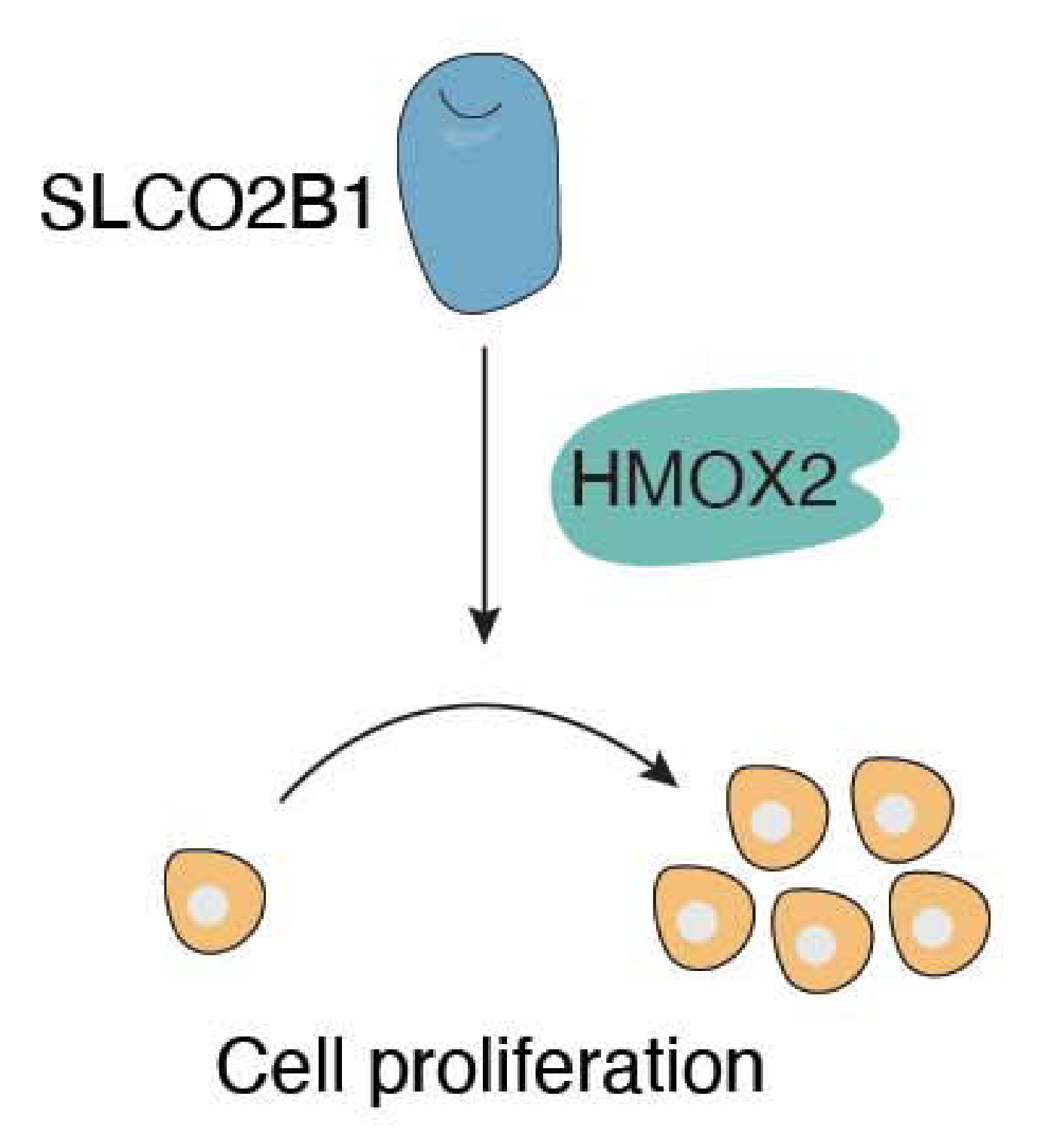

$\mathbf{F}$

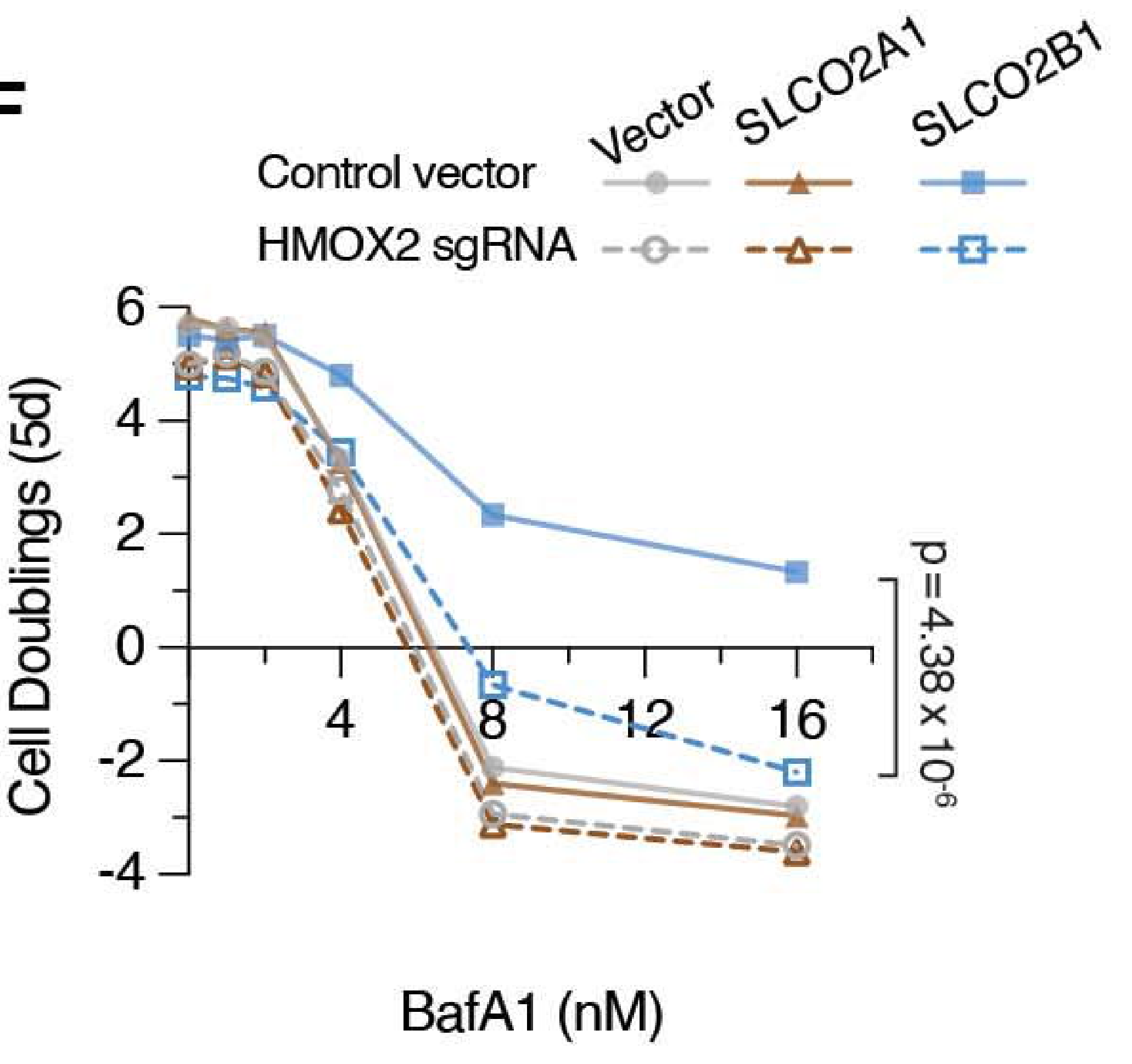

J

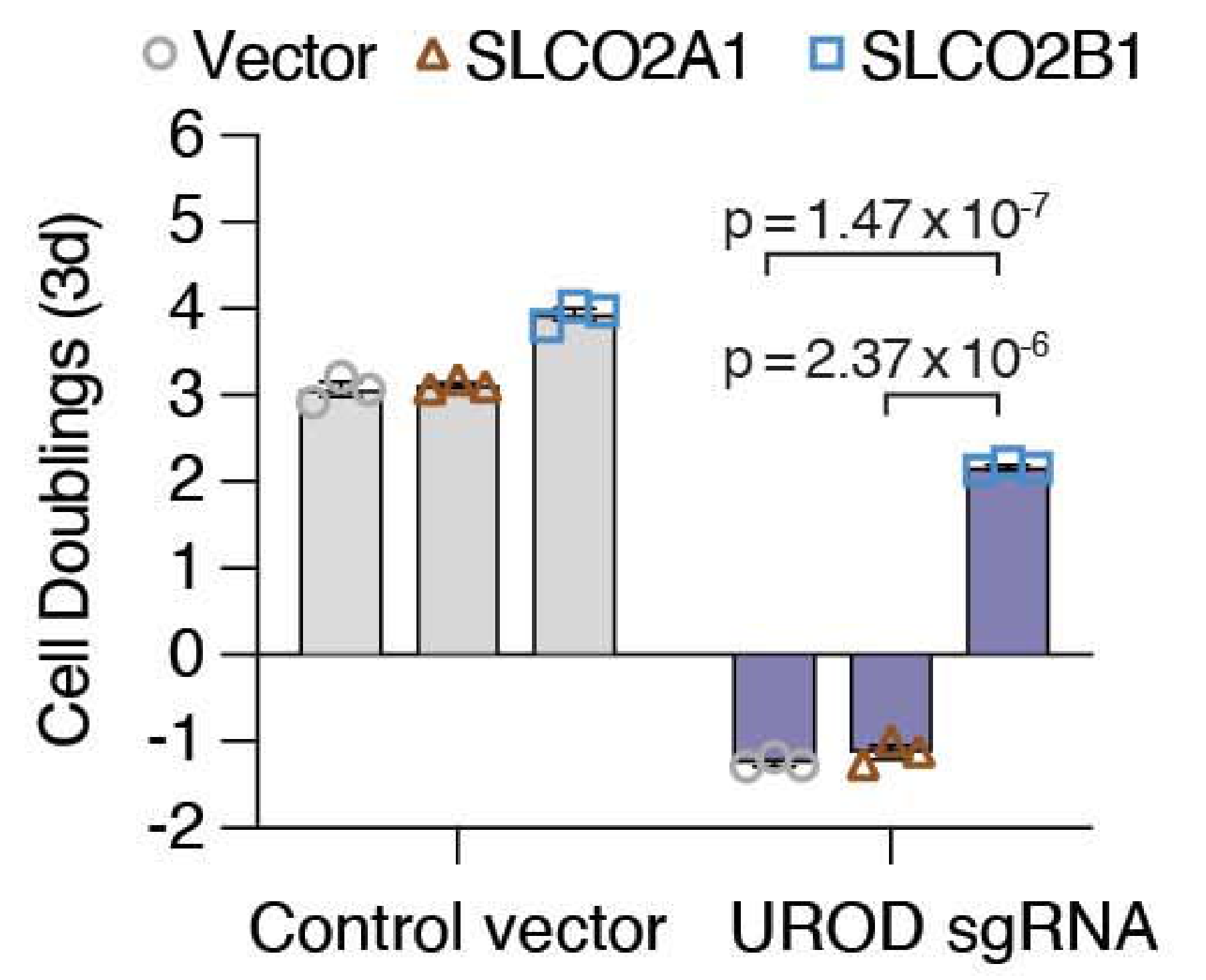

G

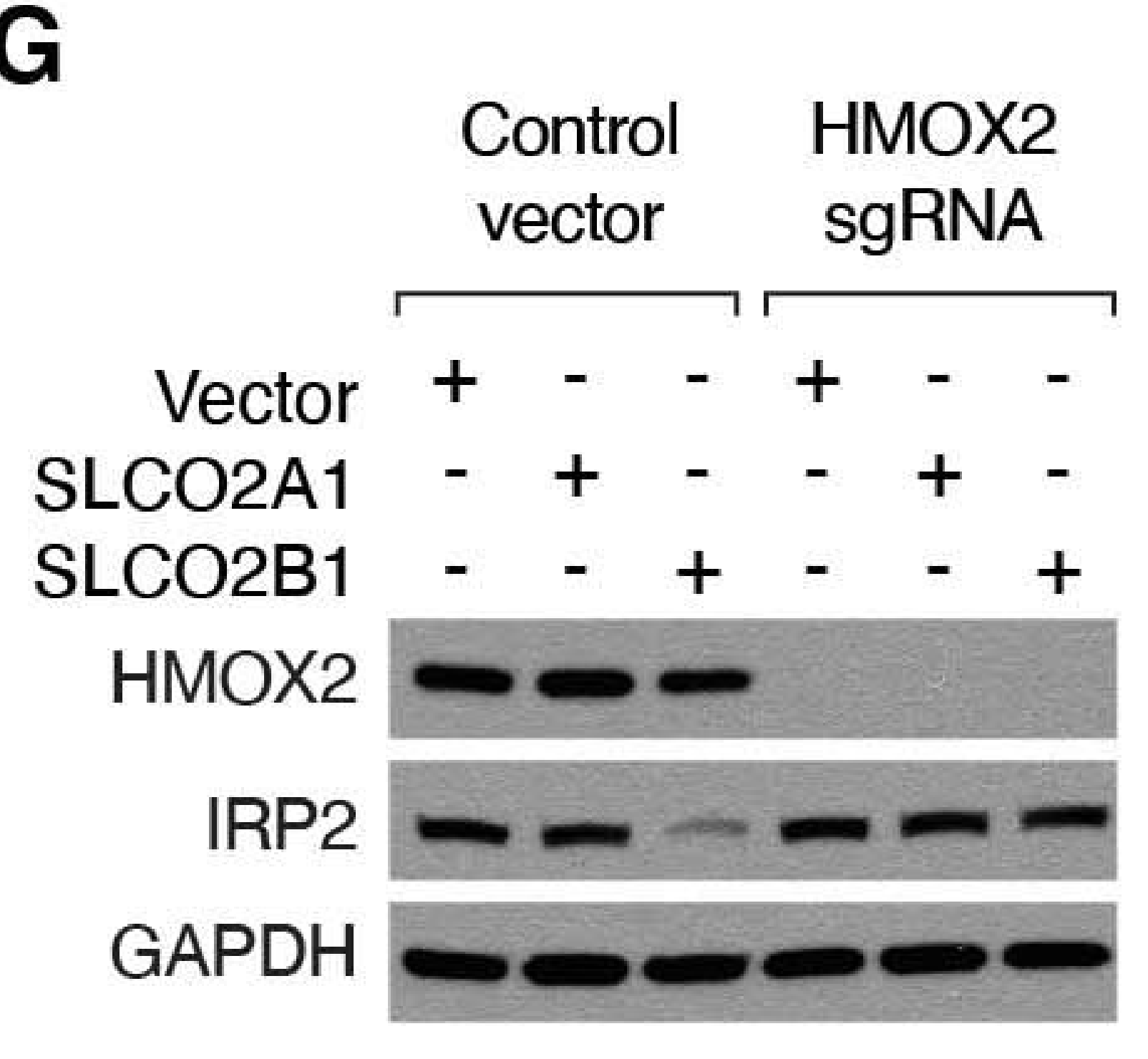

Control vs. SLCO2B1 cDNA

CRISPR screen for differential essentiality

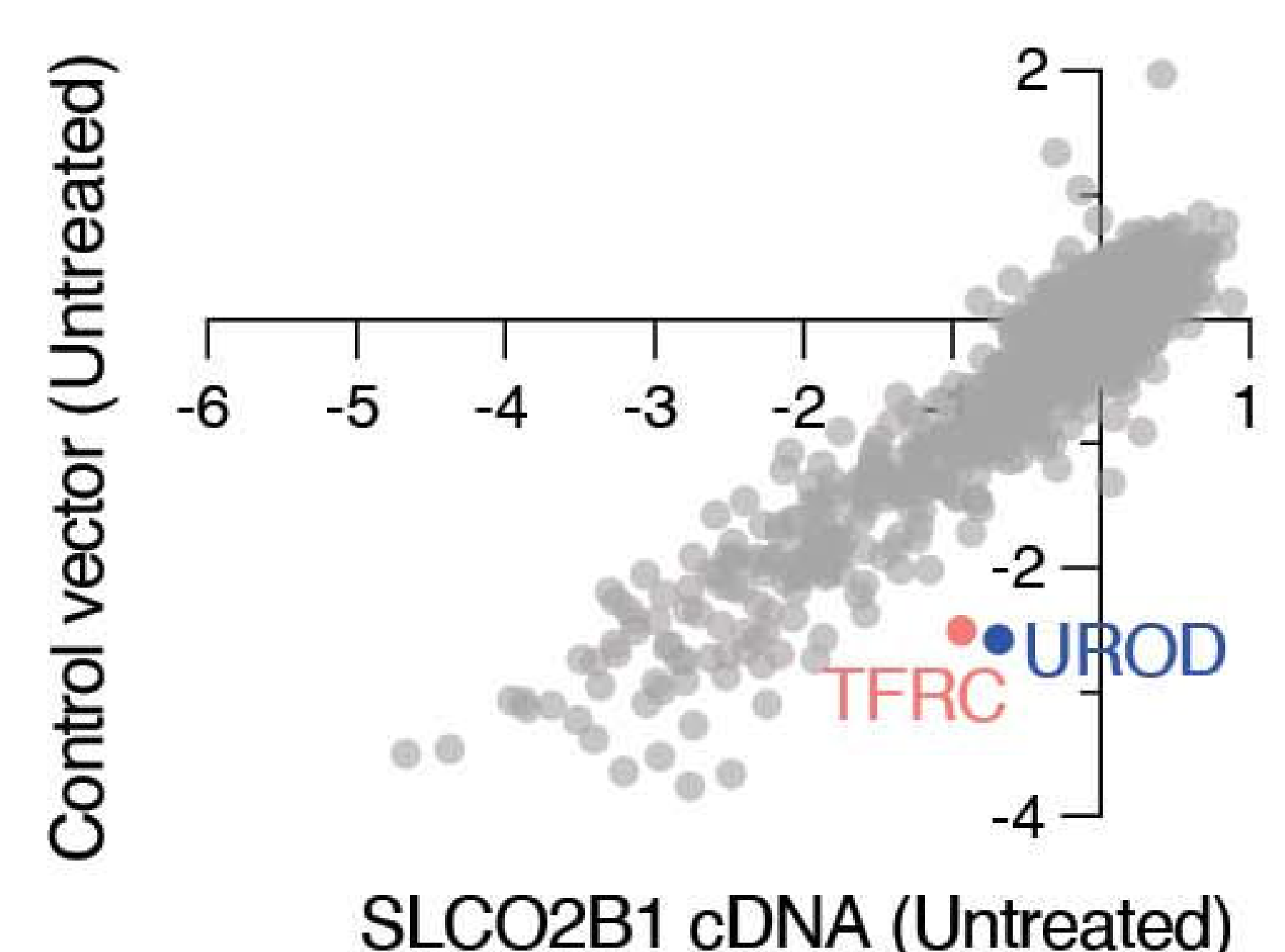

Control vector UROD sgRNA
K

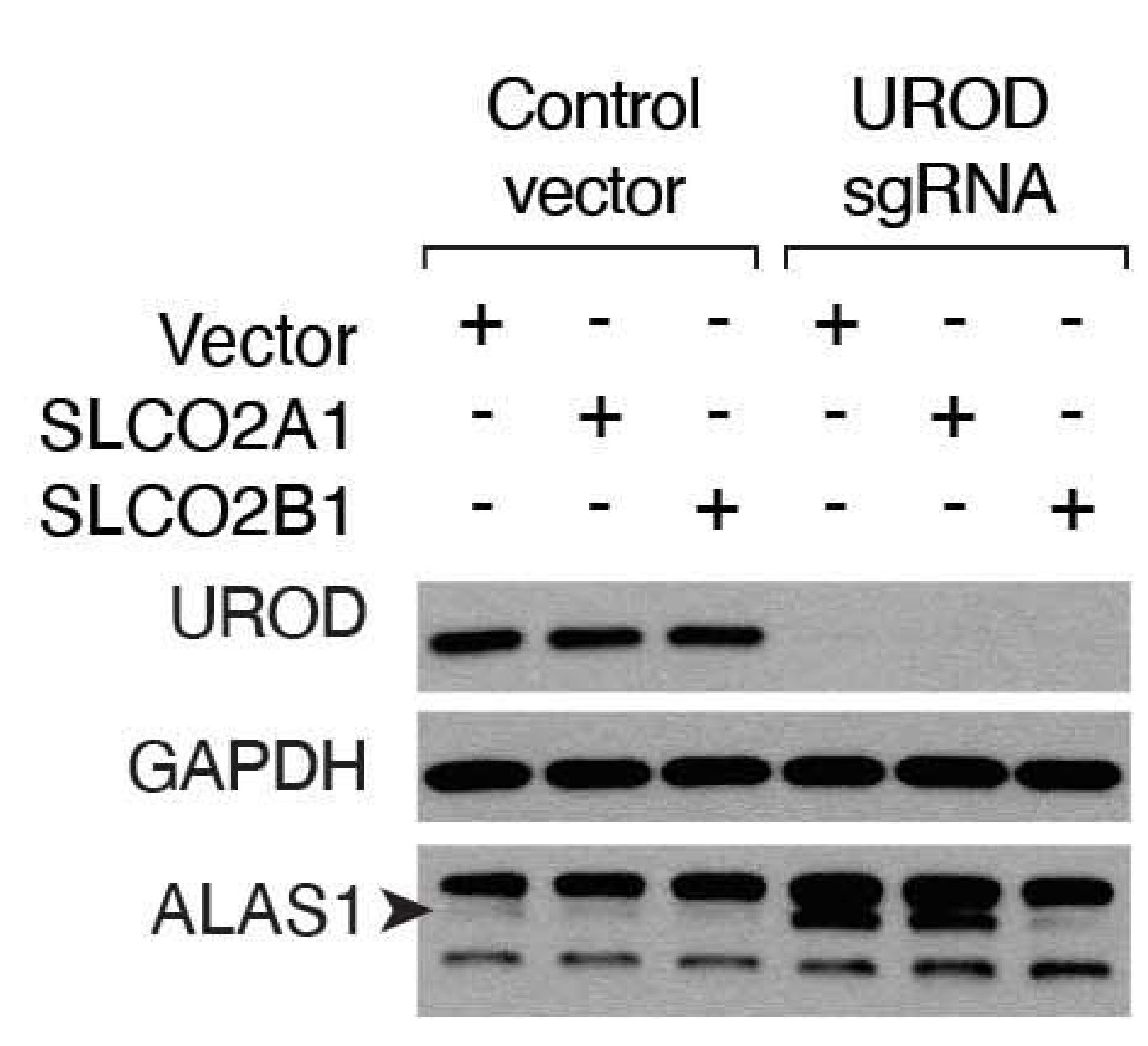


A
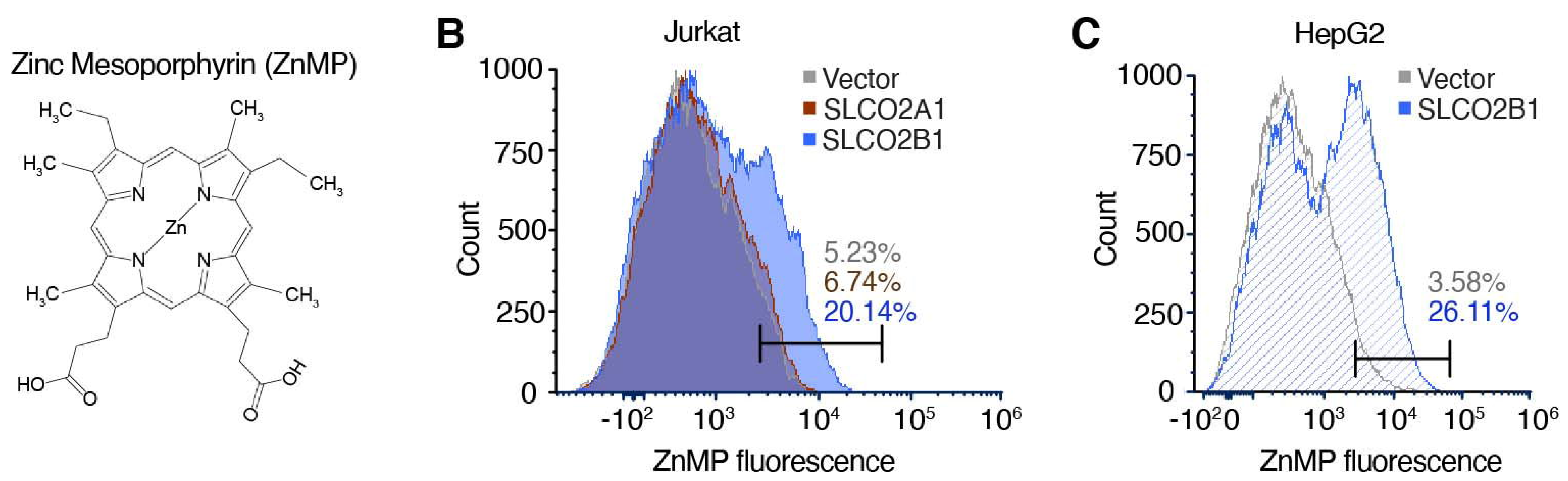

$\mathbf{F}$

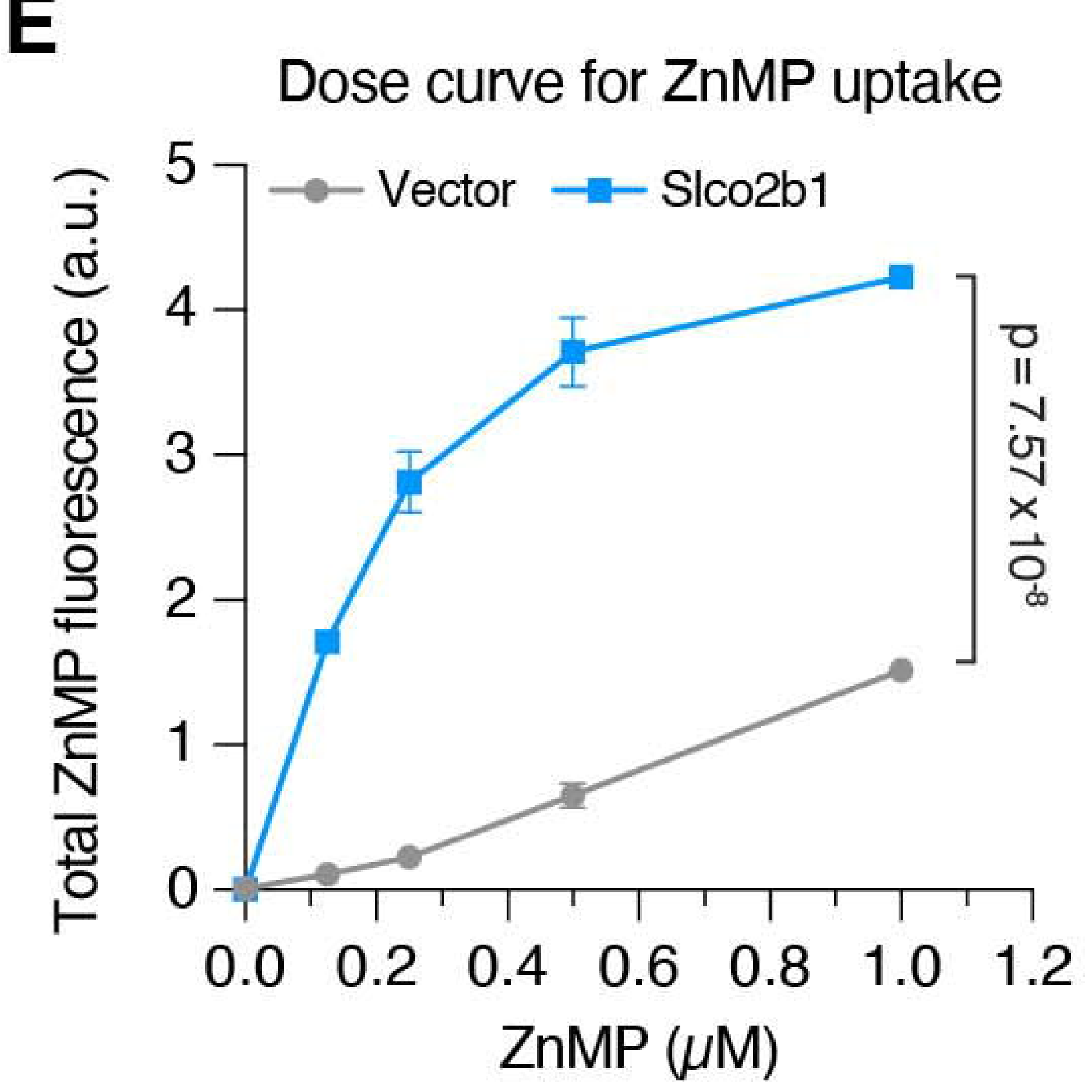

Time course for $1 \mu \mathrm{M}$ ZnMP uptake

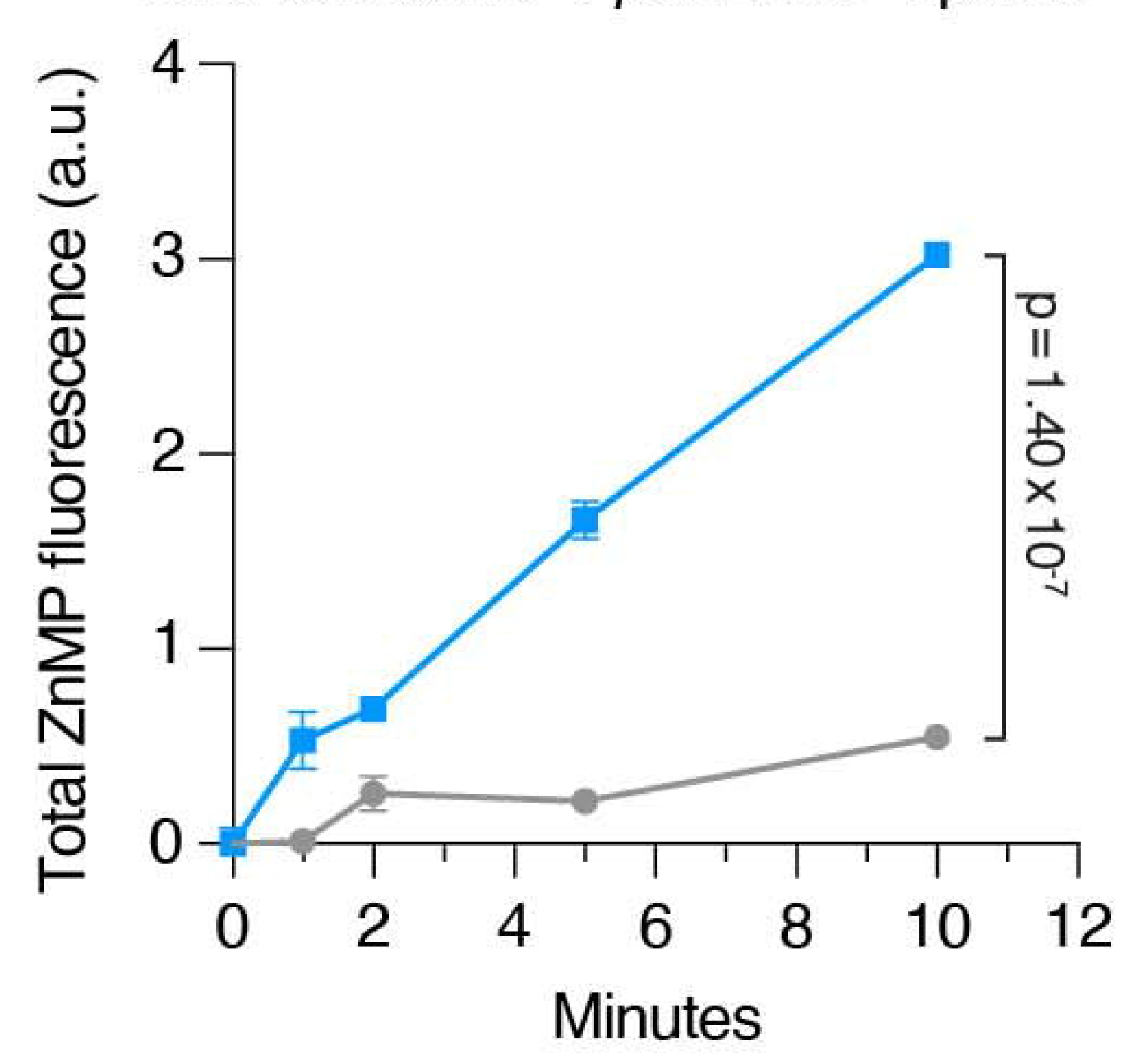

H

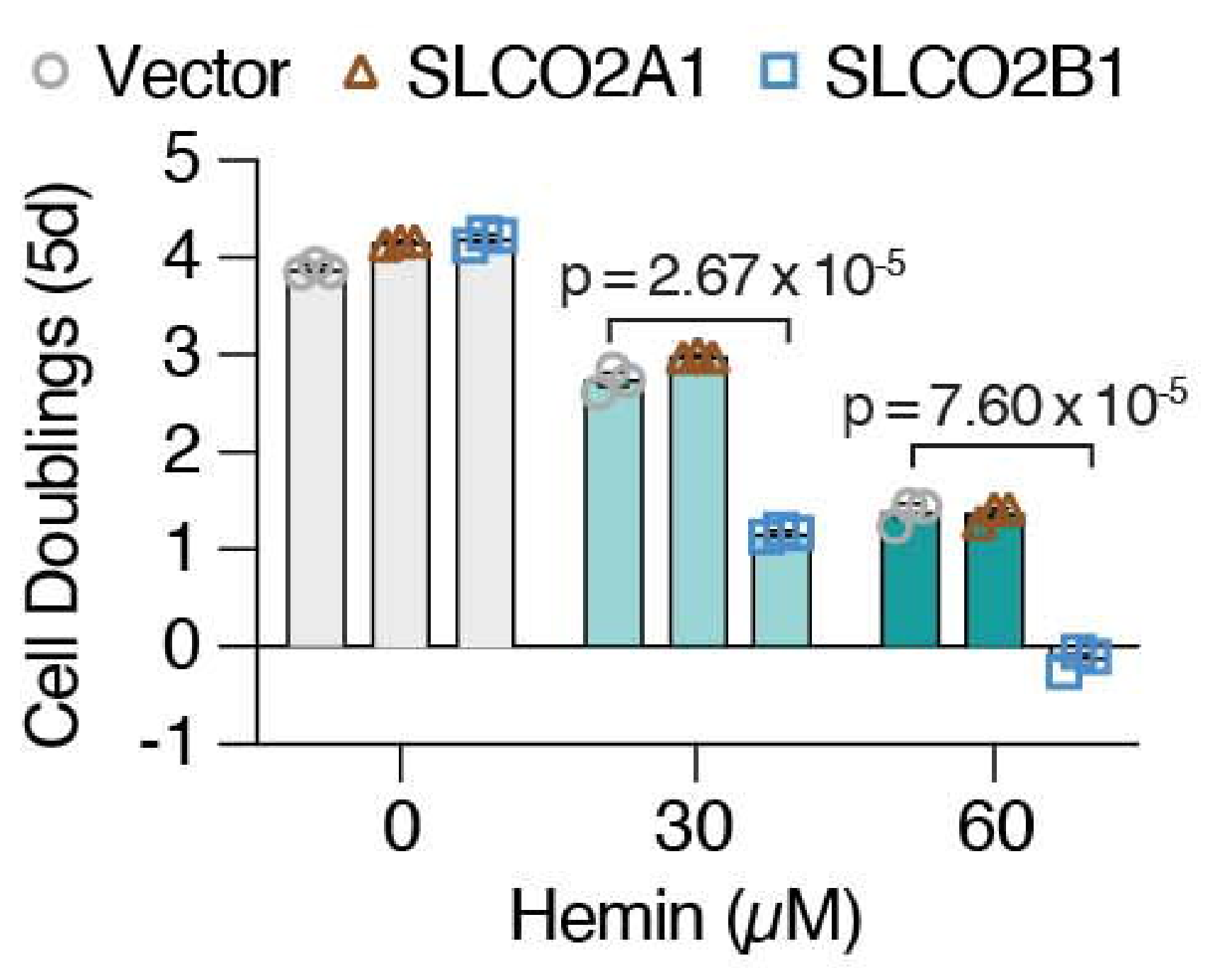

I
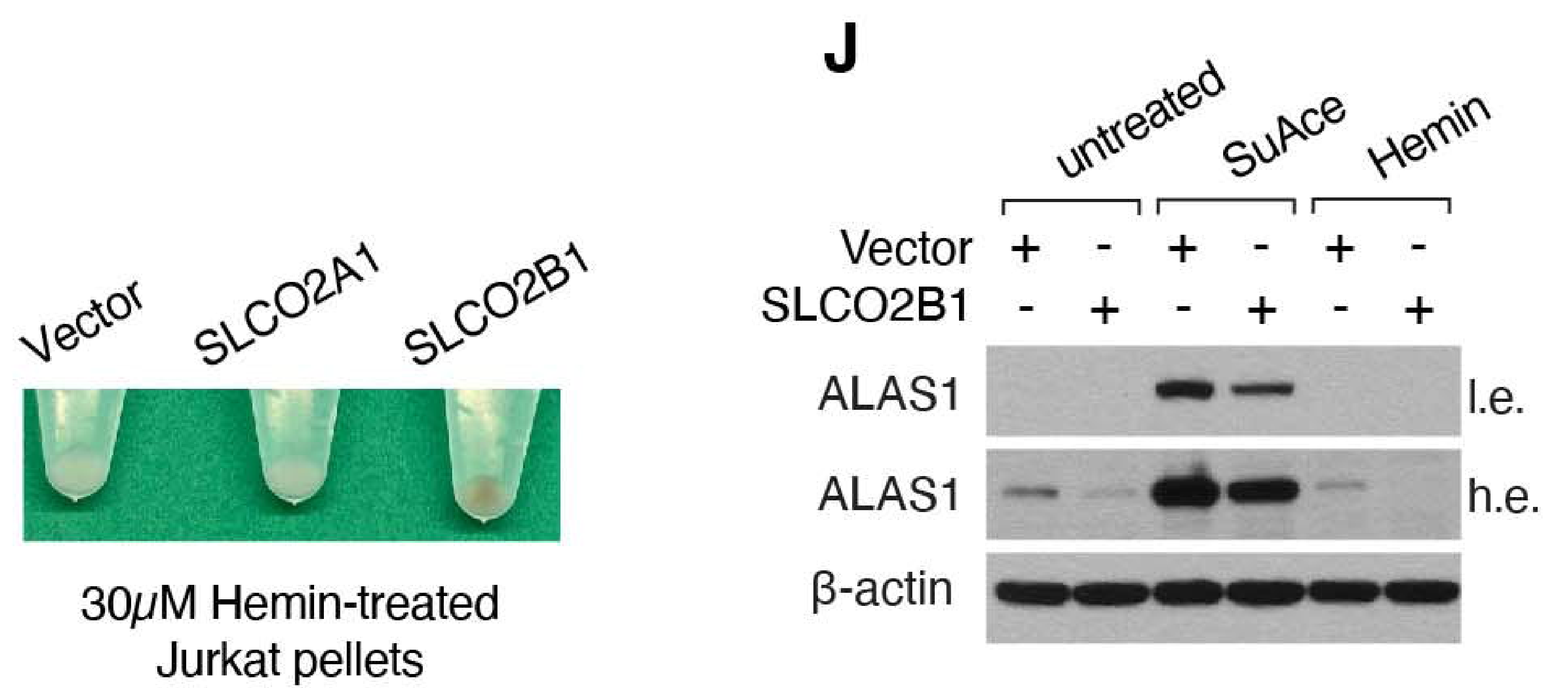

L WT SIco2b1-null

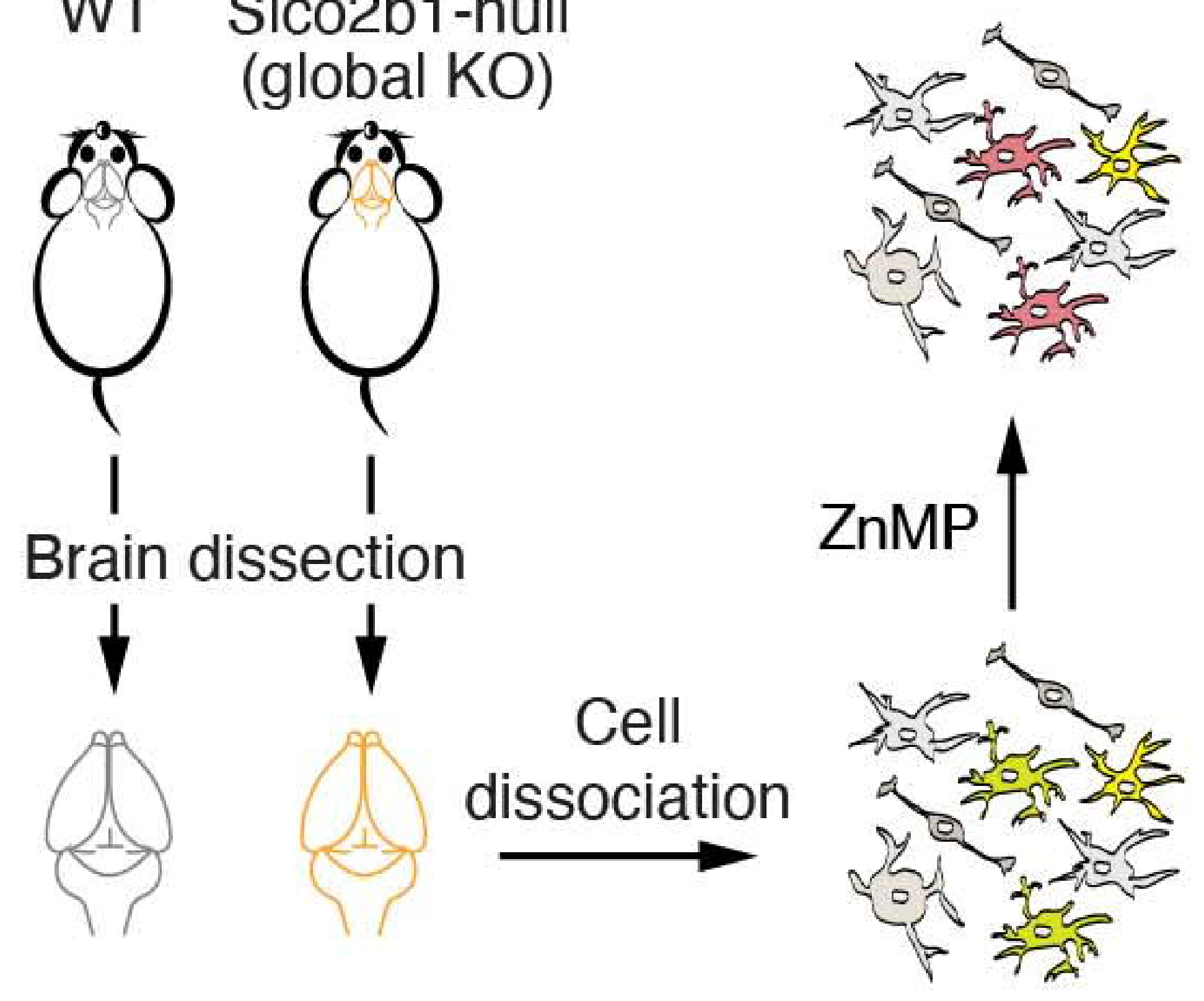

M

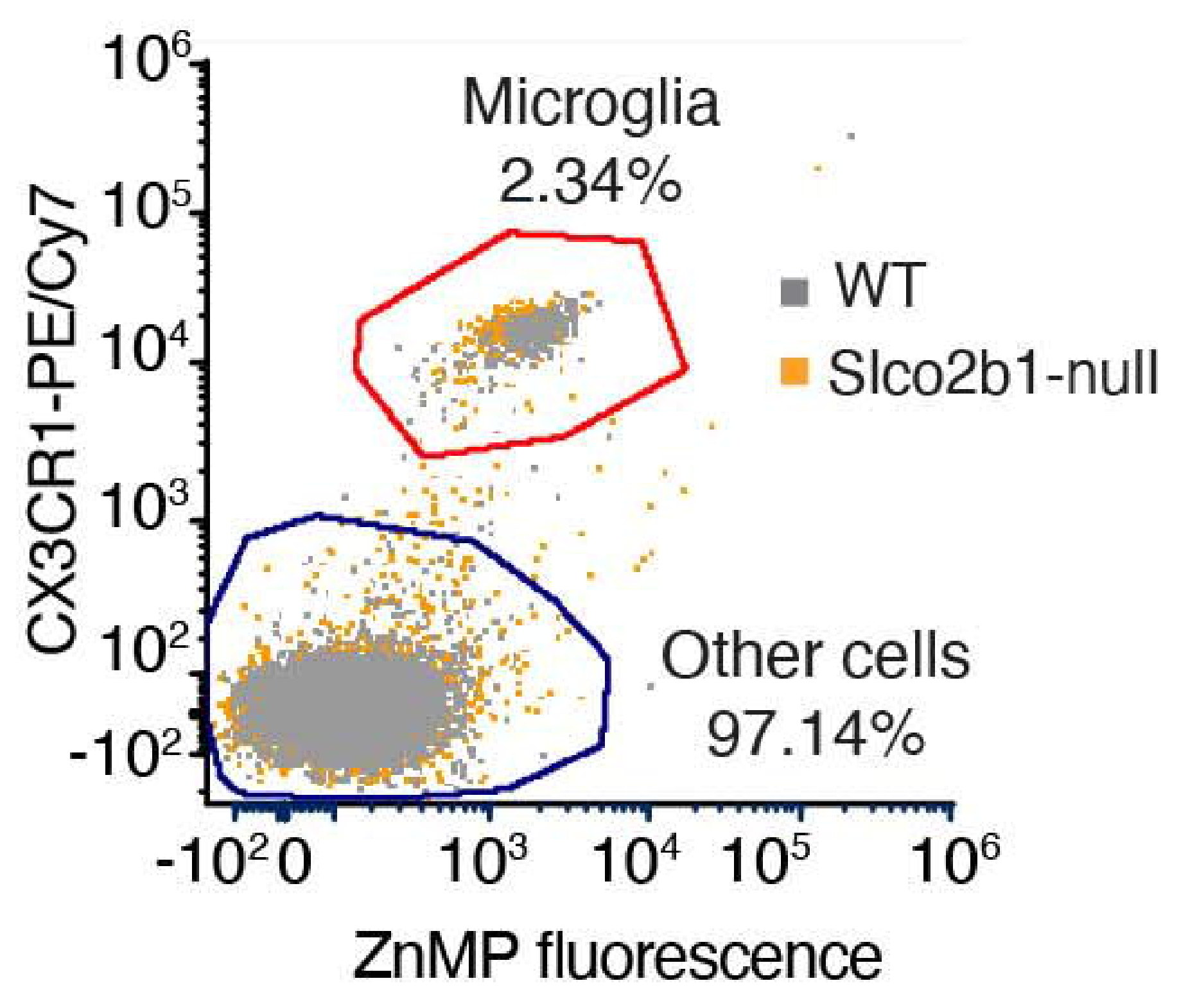

G
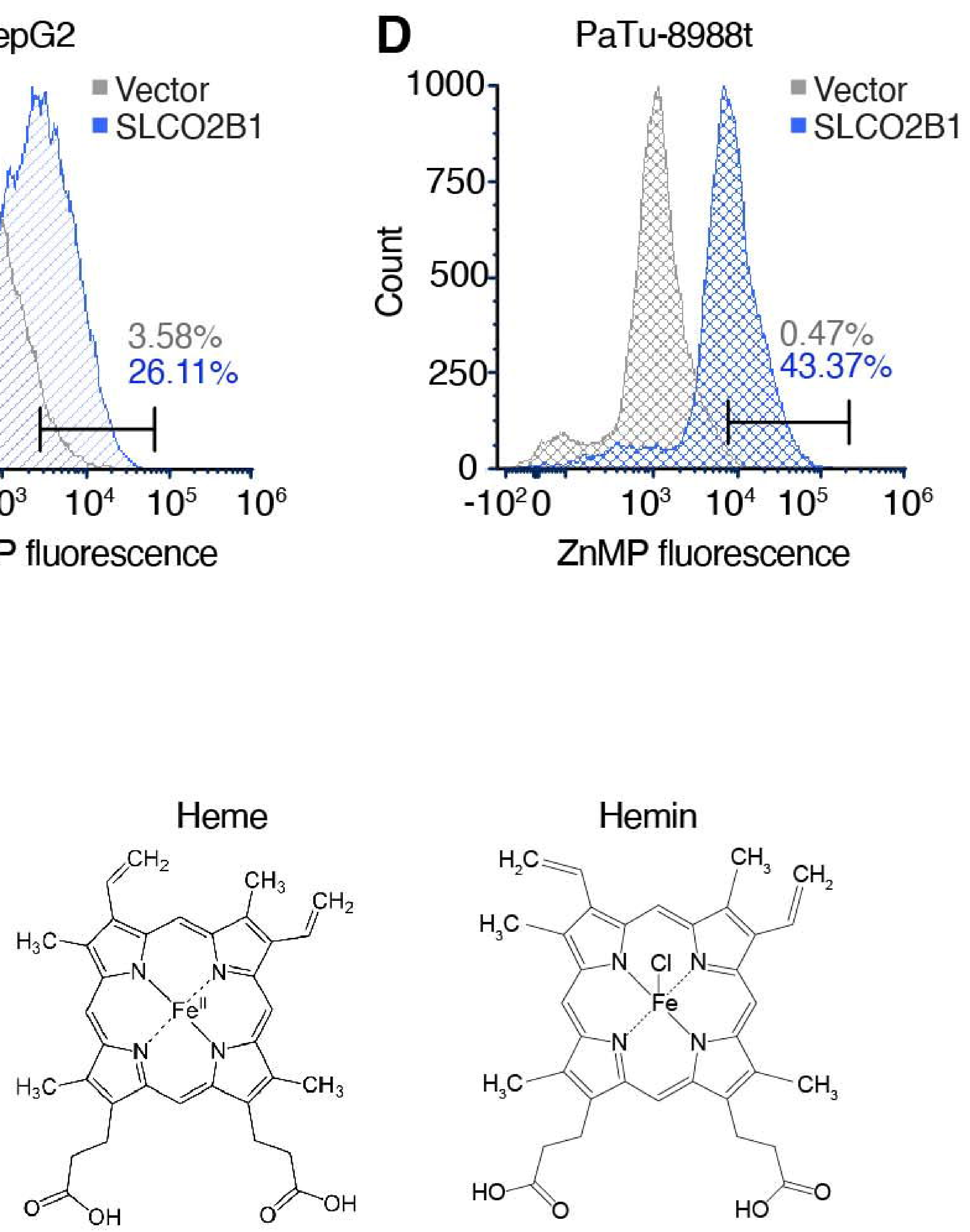

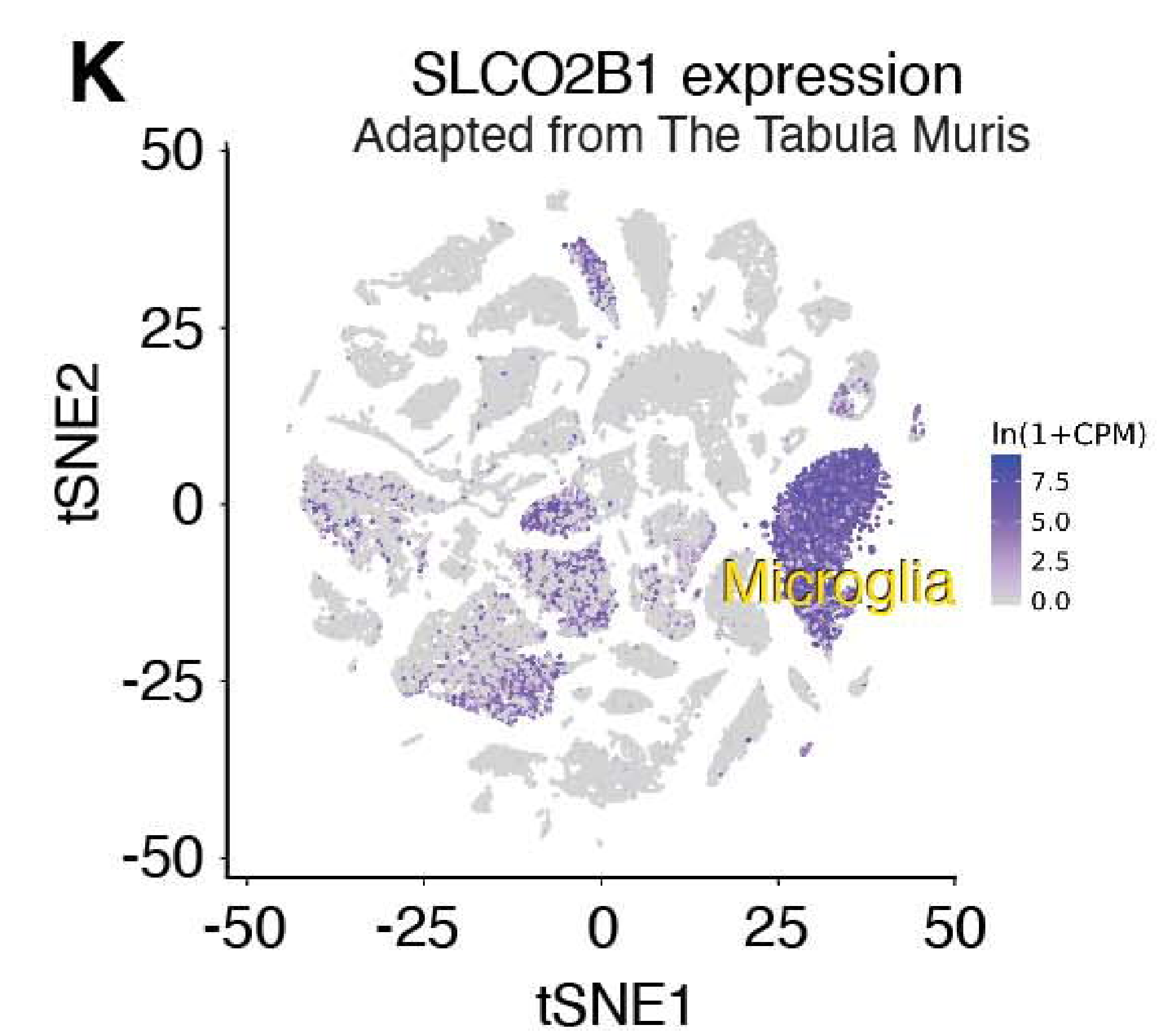

N

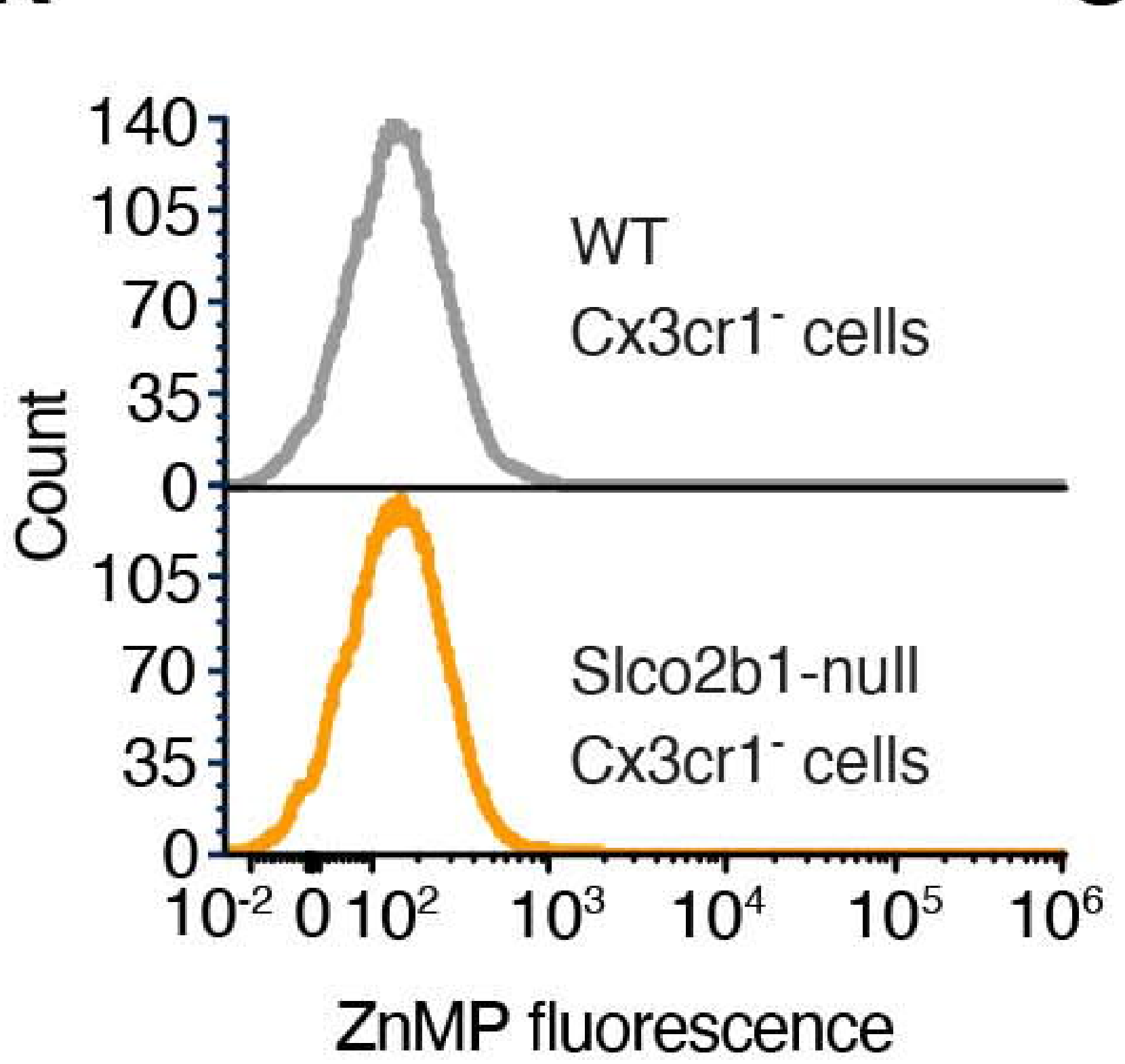

0

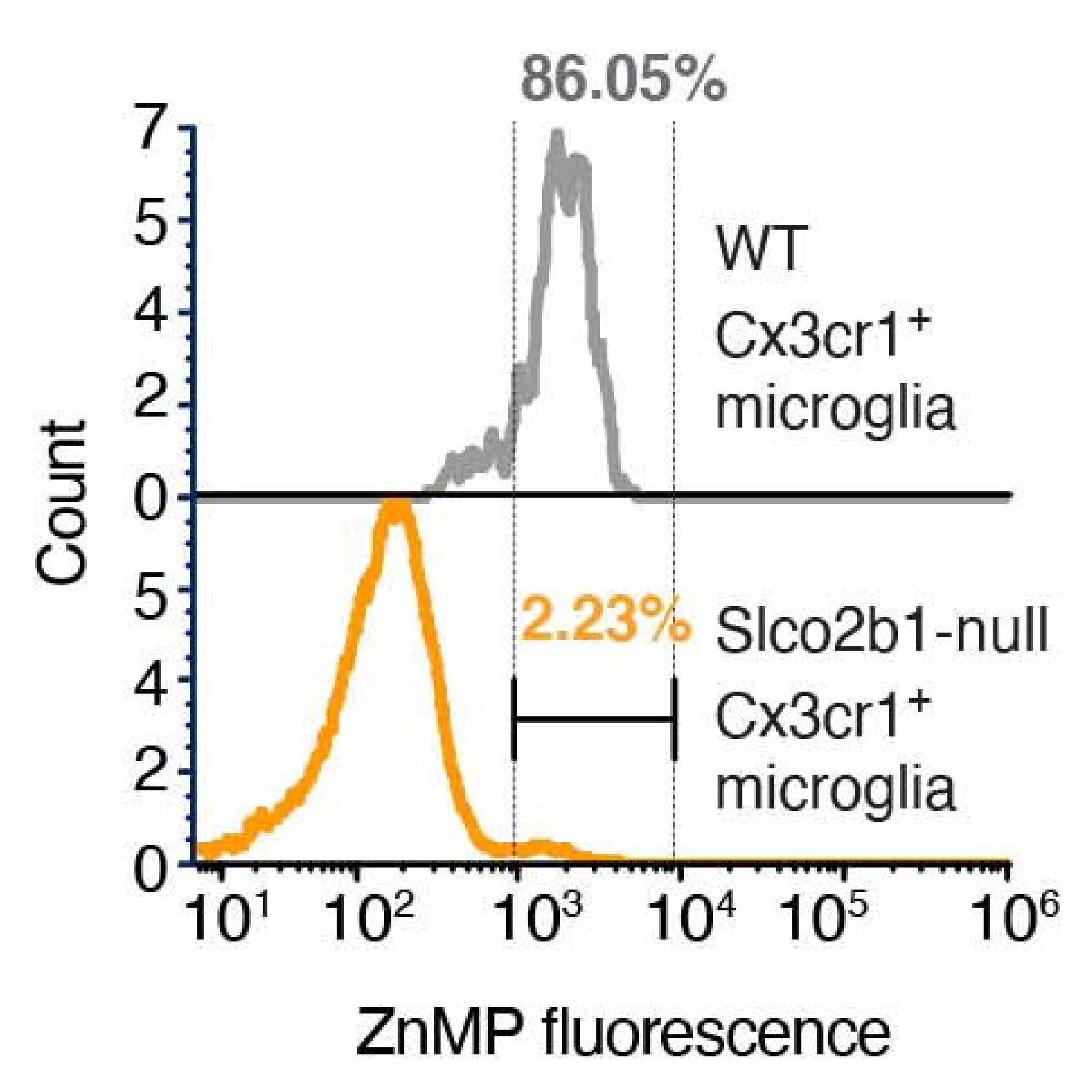

